\title{
The Milstein bipyridyl PNN pincer complex of ruthenium becomes a Noyori- type catalyst under reducing conditions.
}

Louise N. Dawe, ${ }^{\dagger}$ Morteza Karimzadeh-Younjali, ${ }^{\dagger}$ Zengjin Dai, ${ }^{\dagger}$ Eugene Khaskin, ${ }^{*}{ }^{\S}$ Dmitry G. Gusev* ${ }^{\dagger}$

$\dagger$ Department of Chemistry and Biochemistry, Wilfrid Laurier University, Waterloo ON N2L 3C5 Canada.

§Okinawa Institute of Science and Technology, Okinawa, Japan.

Table of Contents

NMR spectra

p. S2-S17

DFT computed energies

p. $\mathrm{S} 18-\mathrm{S} 20$

Crystal Data Collection and Refinement Parameters for 5a, 6, and $\mathbf{7}$

p. S21-S71 




Figure S1. The ${ }^{1} \mathrm{H}$ NMR spectrum of complex 5 in $\mathrm{C}_{6} \mathrm{D}_{6}$.

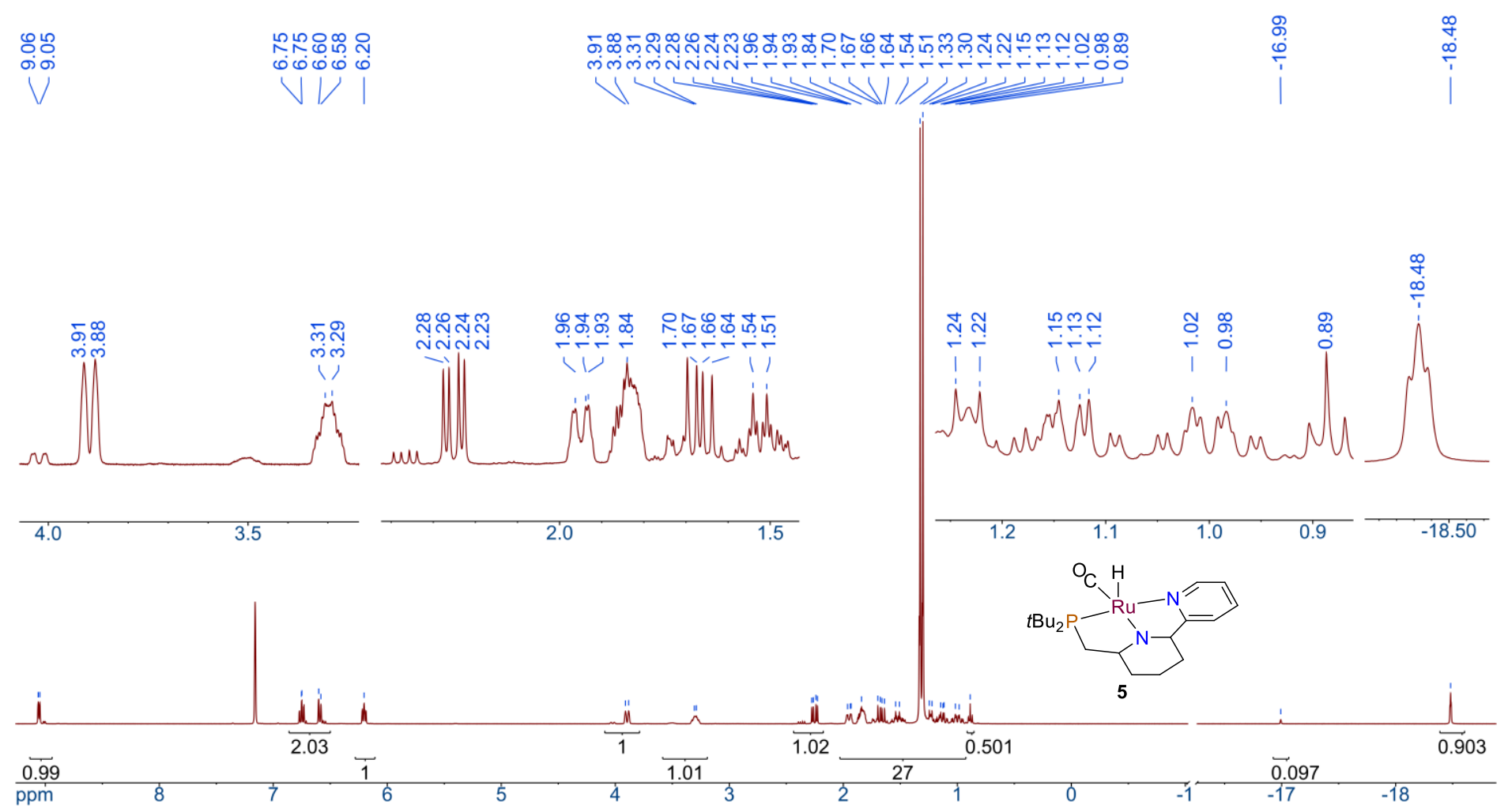

Figure S2. The ${ }^{1} H\left\{{ }^{31} \mathrm{P}\right\}$ NMR spectrum of complex $\mathbf{5}$ in $\mathrm{C}_{6} \mathrm{D}_{6}$. 


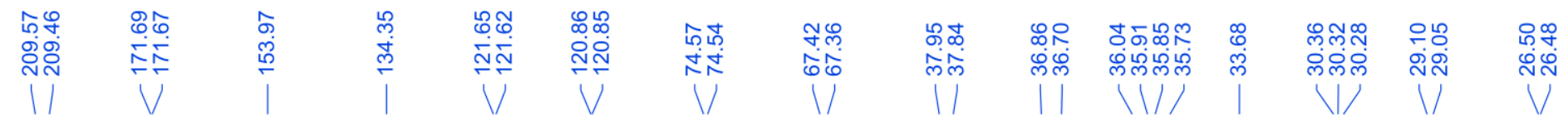
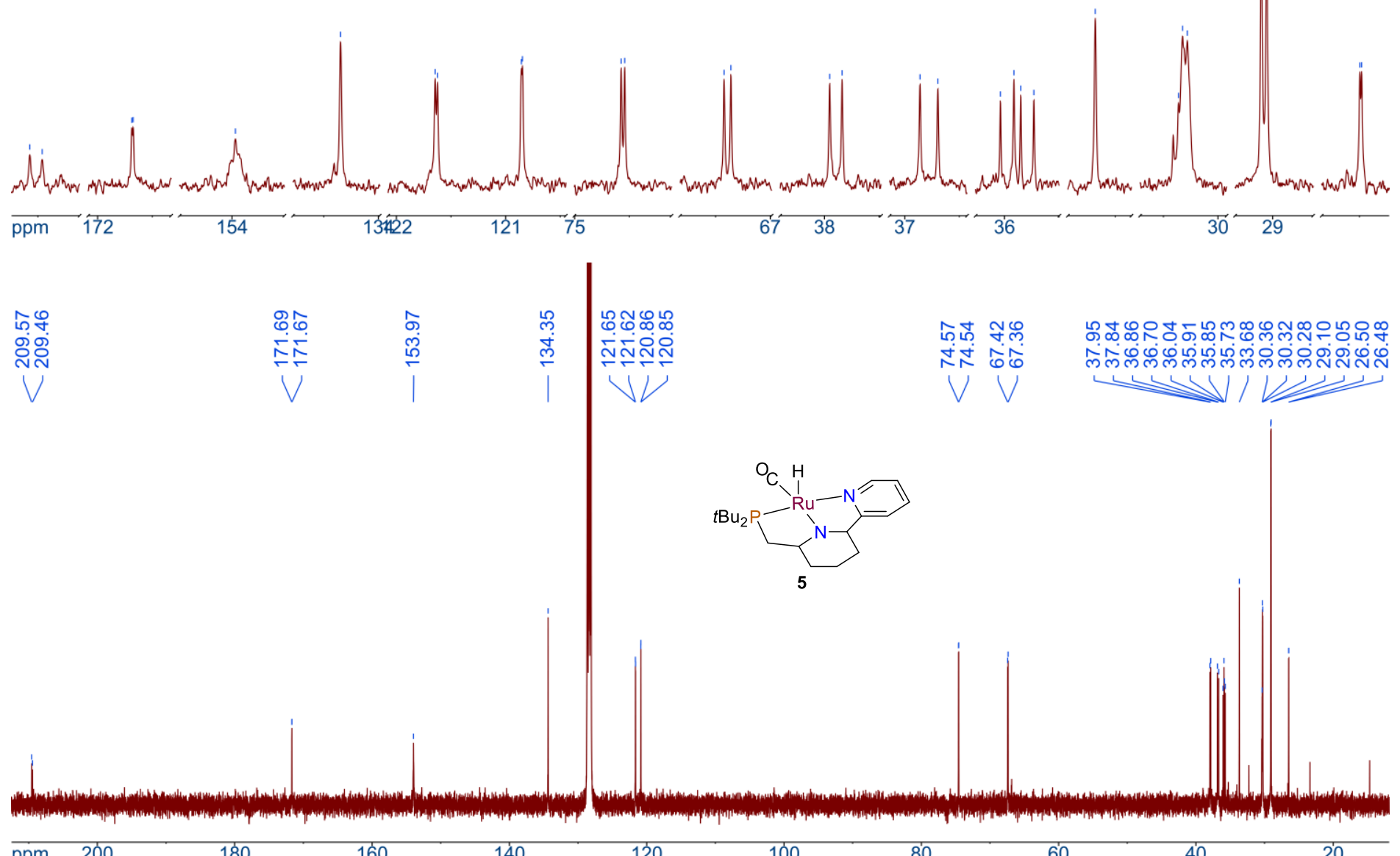

Figure S3. The ${ }^{13} \mathrm{C}\left\{{ }^{1} \mathrm{H}\right\}$ NMR spectrum of 5 in $\mathrm{C}_{6} \mathrm{D}_{6}$; three low-intensity peaks in the $10-33$ ppm region belong to hexane.

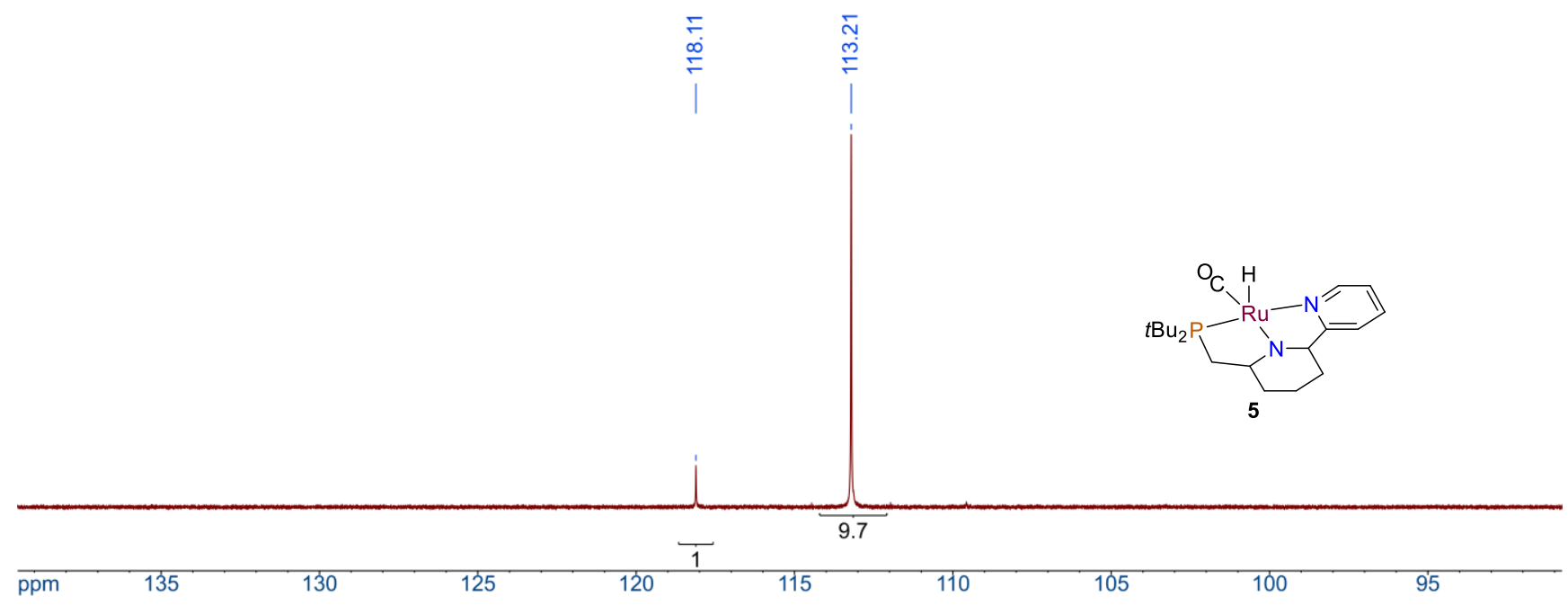

Figure S4. The ${ }^{31} \mathrm{P}\left\{{ }^{1} \mathrm{H}\right\}$ NMR spectrum of complex $\mathbf{5}$ in $\mathrm{C}_{6} \mathrm{D}_{6}$. 


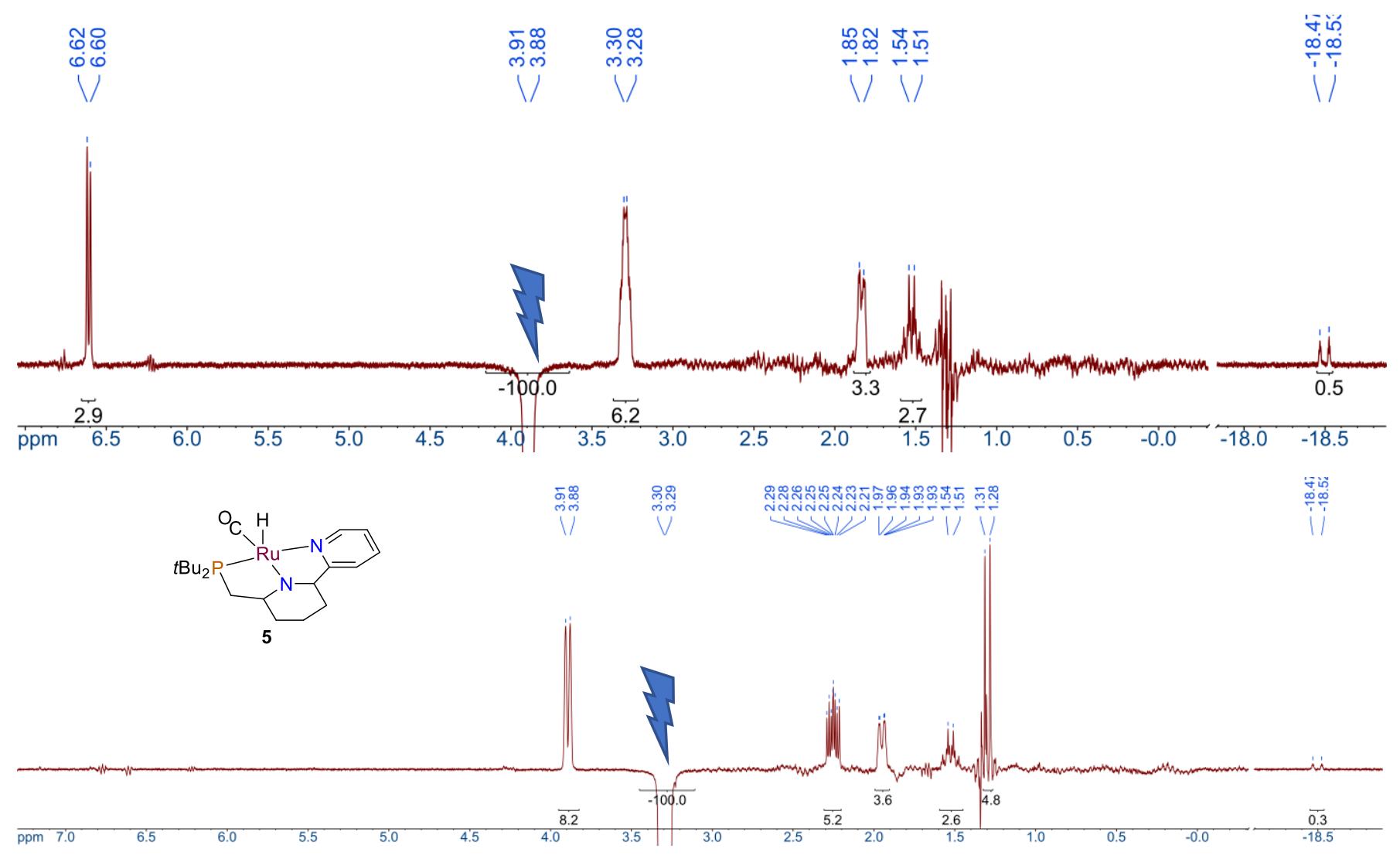

Figure S5. ${ }^{1} \mathrm{H}$ NOE difference NMR spectra demonstrating the effect of irradiating the NCH resonances of complex $\mathbf{5}$.

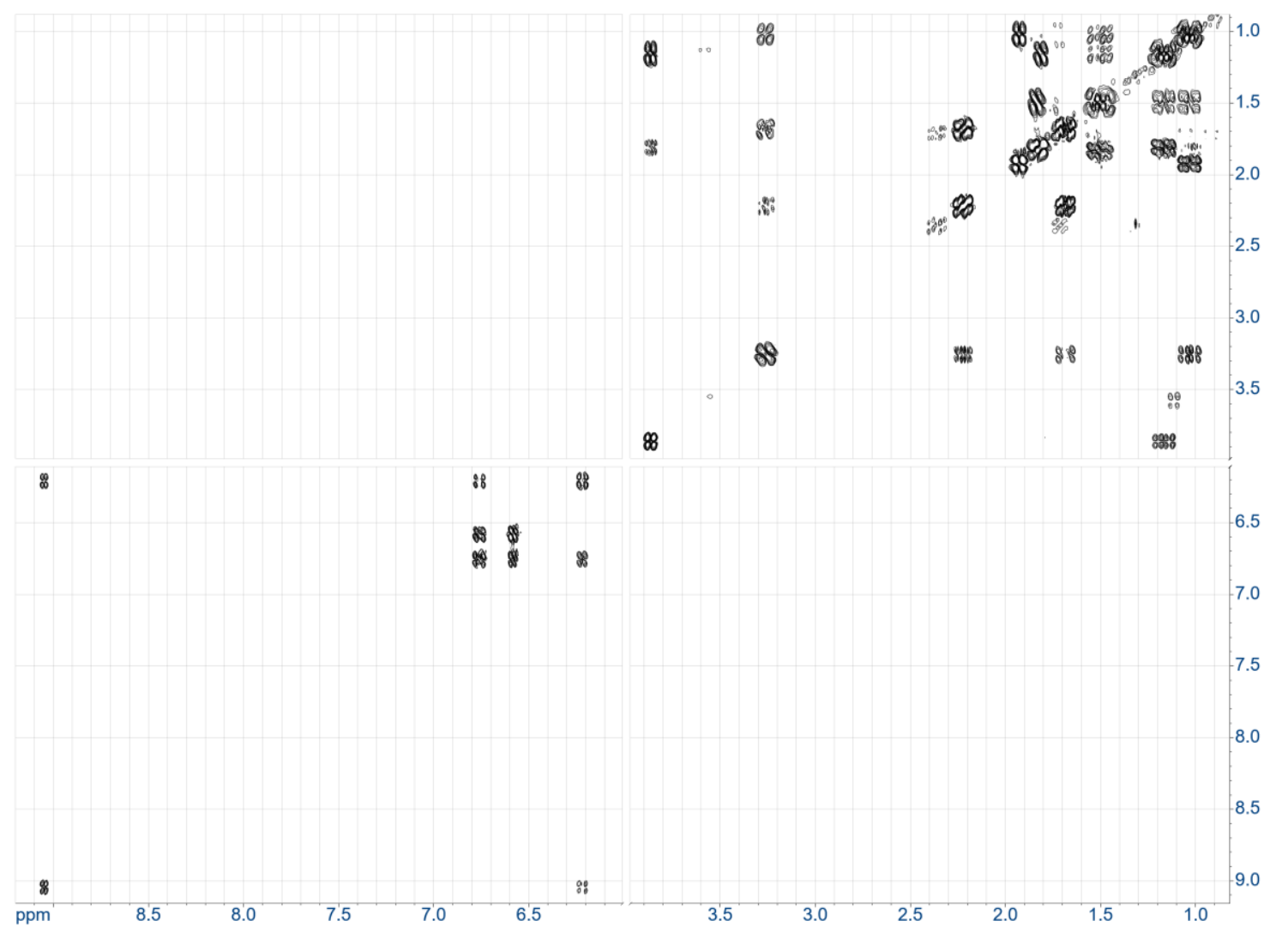

Figure S6. A COSY spectrum of complex 5. 




Figure S7. The ${ }^{1} \mathrm{H}$ NMR spectrum of complex 6 in $\mathrm{C}_{6} \mathrm{D}_{6}$; the intense signal at 7.16 is the solvent resonance, flanked by the ${ }^{13} \mathrm{C}$ satellites.

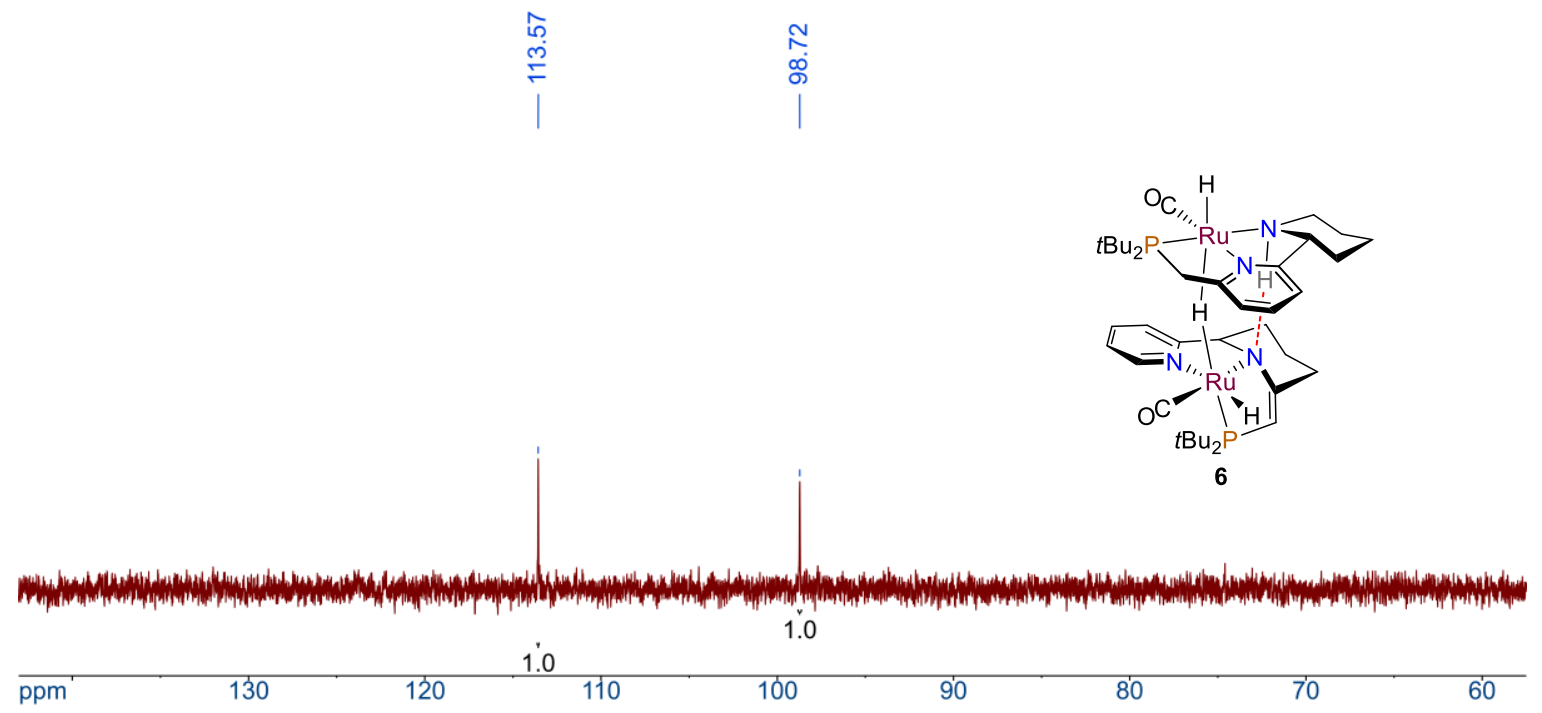

Figure S8. The ${ }^{31} \mathrm{P}\left\{{ }^{1} \mathrm{H}\right\}$ NMR spectrum of complex 6 in $\mathrm{C}_{6} \mathrm{D}_{6}$. 


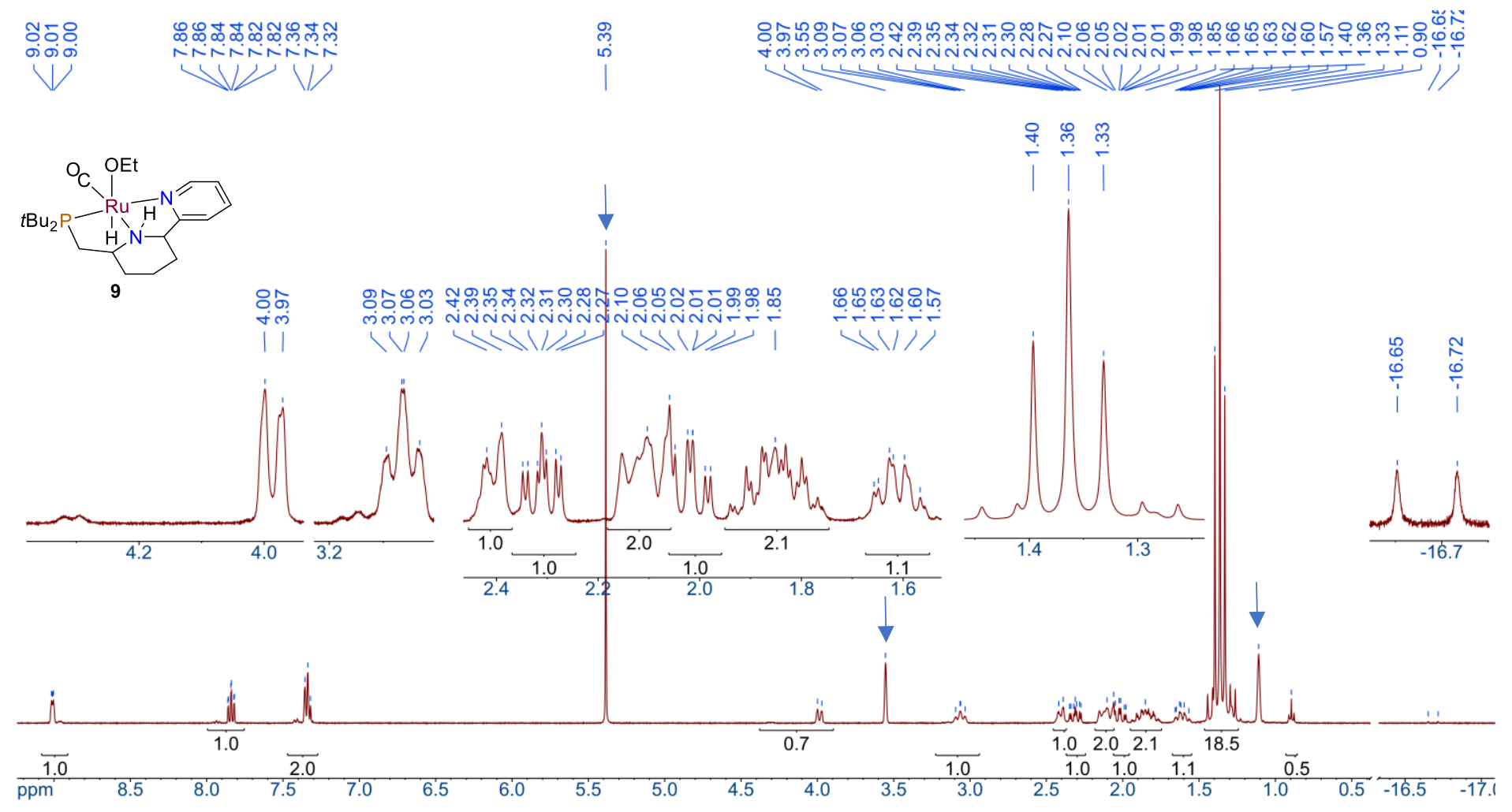

Figure S9. The ${ }^{1} \mathrm{H}$ NMR spectrum of complex 9 prepared by dissolving $26 \mathrm{mg}$ of $\mathbf{5} \mathrm{in} 0.67 \mathrm{ml}$ of ethanol-d6. Residual solvent resonances are indicated by the arrows. A trace of hexane was present in $\mathbf{5}$.

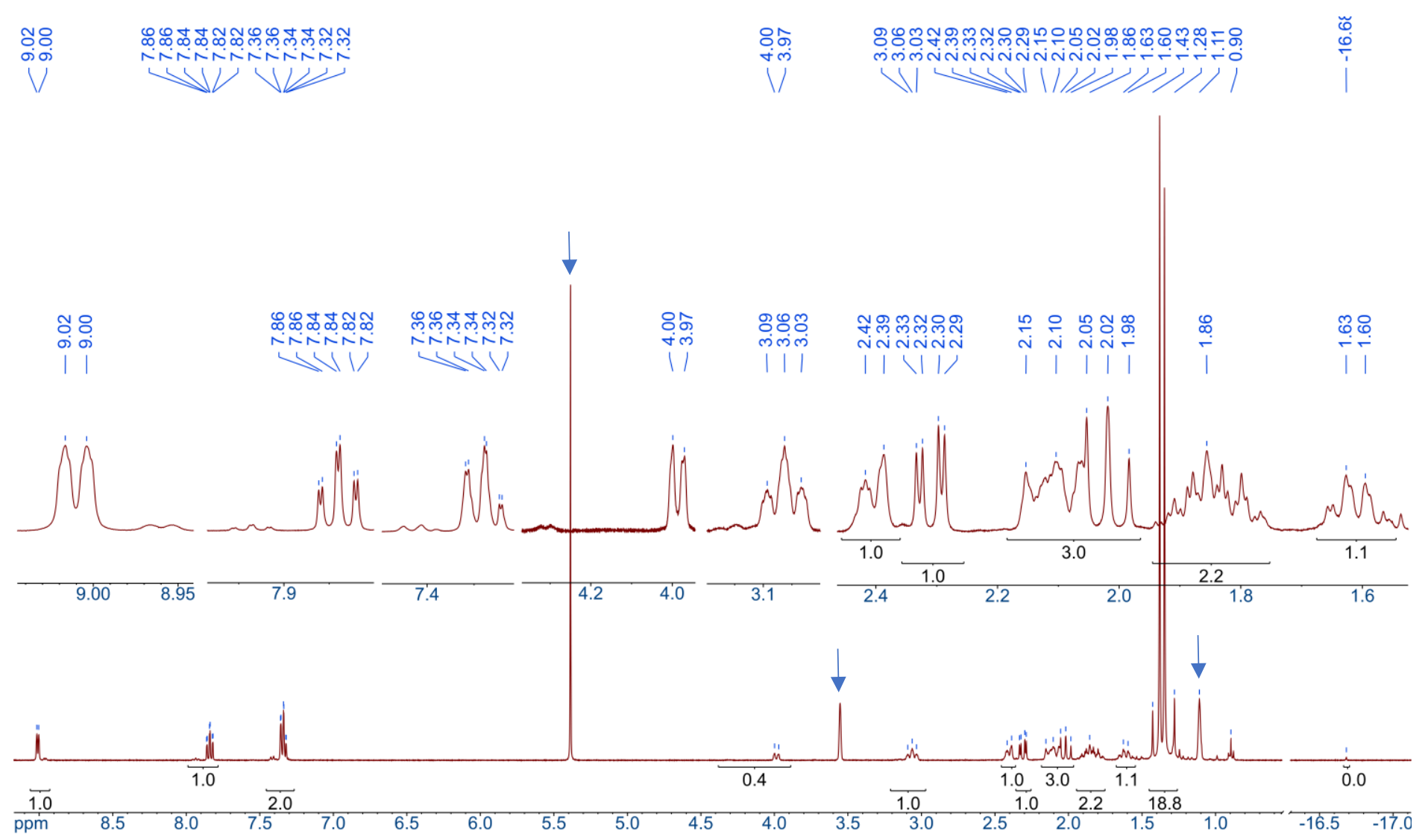

Figure S10. The ${ }^{1} \mathrm{H}\left\{{ }^{31} \mathrm{P}\right\}$ NMR spectrum of complex 9 prepared by dissolving $26 \mathrm{mg}$ of $\mathbf{5}$ in $0.67 \mathrm{ml}$ of ethanol-d $\mathrm{d}_{6}$. Residual solvent resonances are indicated by the arrows. A trace of hexane was present in $\mathbf{5}$ 


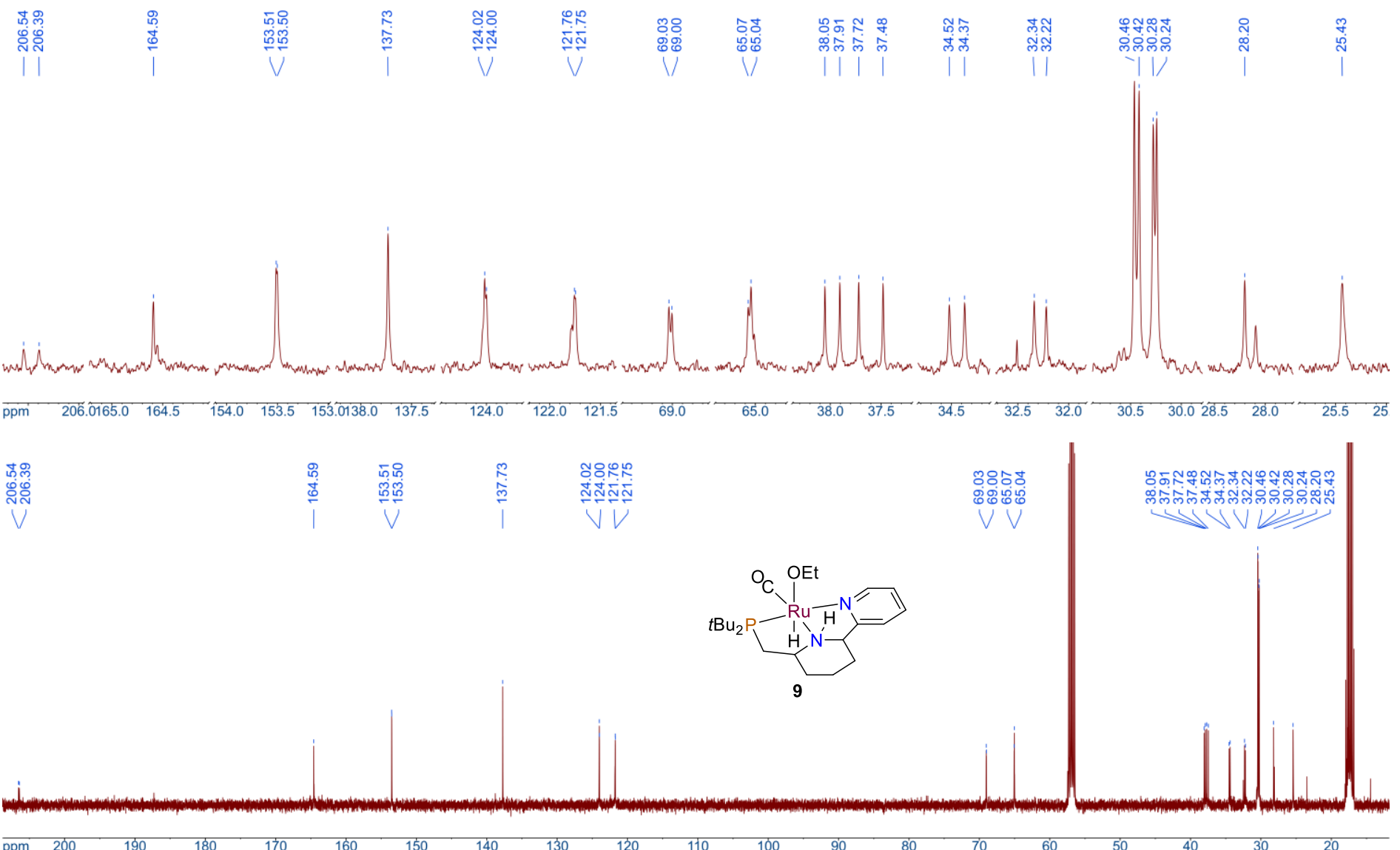

Figure S11. The ${ }^{13} \mathrm{C}\left\{{ }^{1} \mathrm{H}\right\}$ NMR spectrum of 9 prepared by dissolving $26 \mathrm{mg}$ of $\mathbf{5}$ in $0.67 \mathrm{ml}$ of ethanol-d $\mathrm{d}_{6}$; three low-intensity peaks in the $10-33$ ppm region belong to hexane that was present in $\mathbf{5}$.

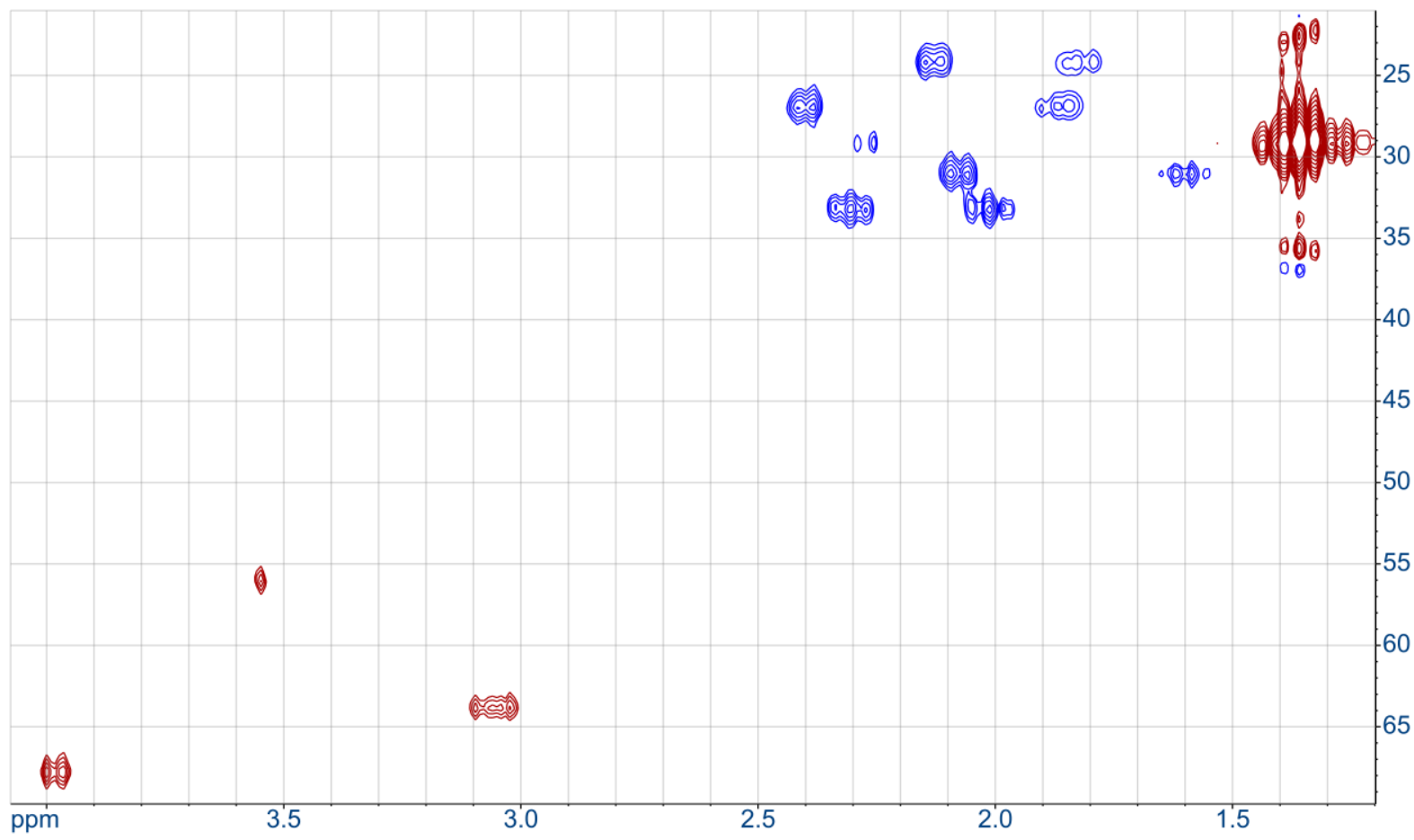

Figure S12. A fragment of the phase-sensitive ${ }^{1} \mathrm{H} /{ }^{13} \mathrm{C}$ correlated HMQC 2D NMR spectrum of 9 in ethanol-d6. 


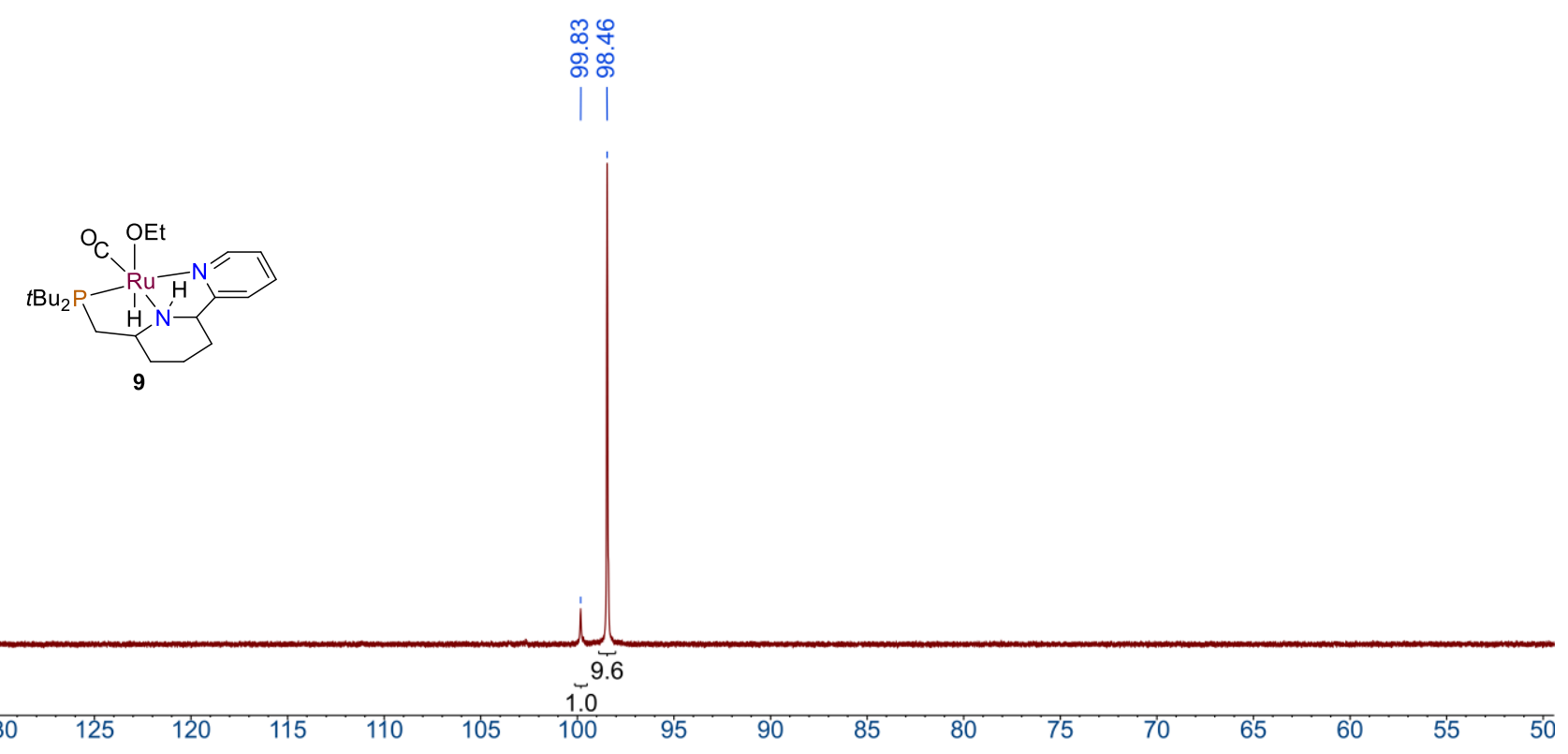

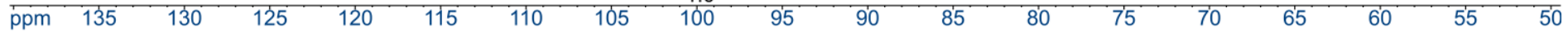

Figure S13. The ${ }^{31} \mathrm{P}\left\{{ }^{1} \mathrm{H}\right\}$ NMR spectrum of 9 prepared by dissolving $26 \mathrm{mg}$ of $\mathbf{5} \mathrm{in} 0.67 \mathrm{ml}$ of ethanol-d 6.

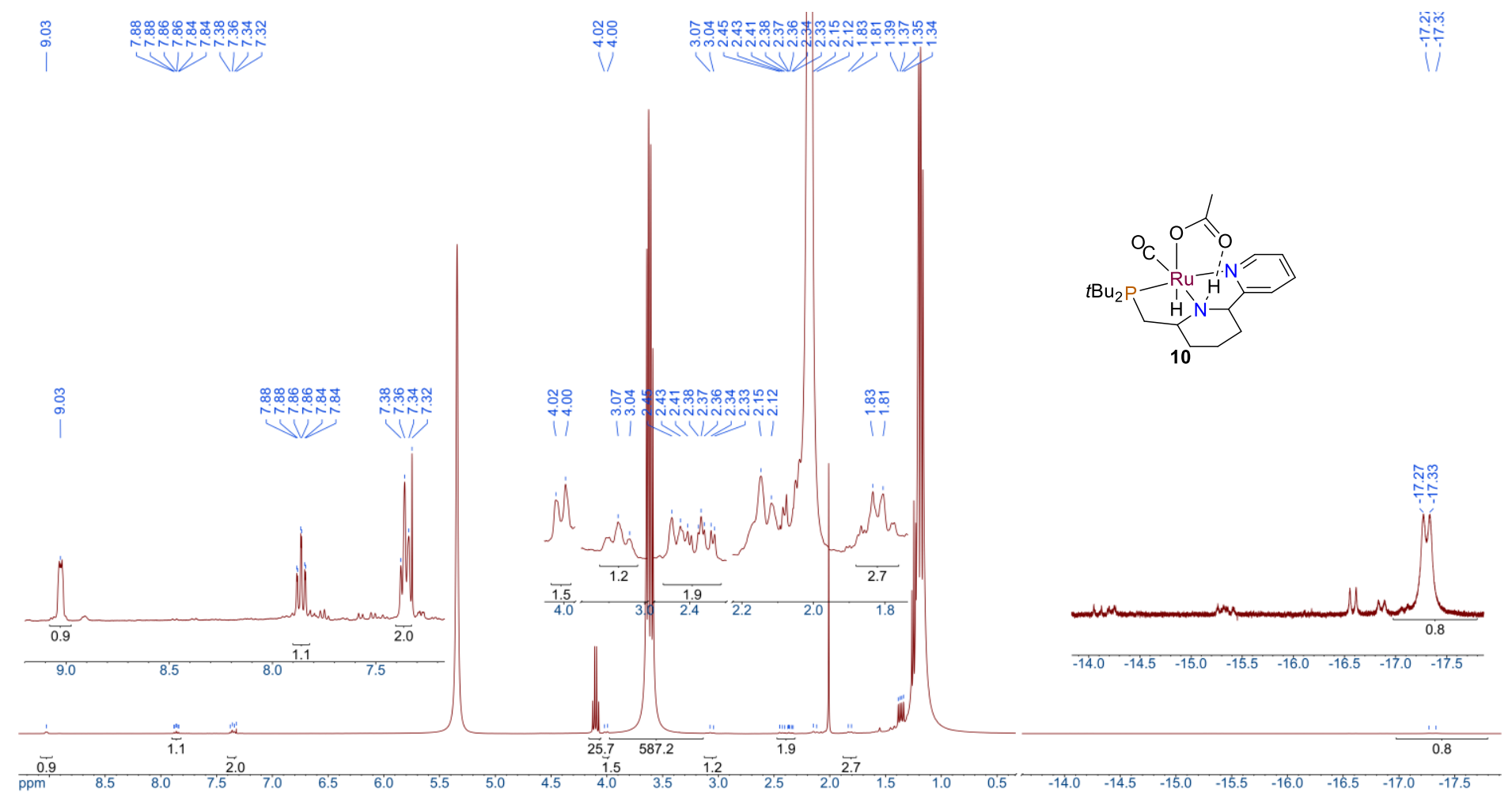

Figure S14. The ${ }^{1} \mathrm{H}$ NMR spectrum of the solution formed upon heating $20 \mathrm{mg}$ of $2 \mathrm{in} 0.65 \mathrm{ml}$ of EtOH at $80{ }^{\circ} \mathrm{C}$ for $2 \mathrm{~h}$. This spectrum displays the resonances of ethyl acetate produced by dehydrogenative coupling of ethanol. A trace amount of benzene (present in 2) is seen at $7.32 \mathrm{ppm}$. 


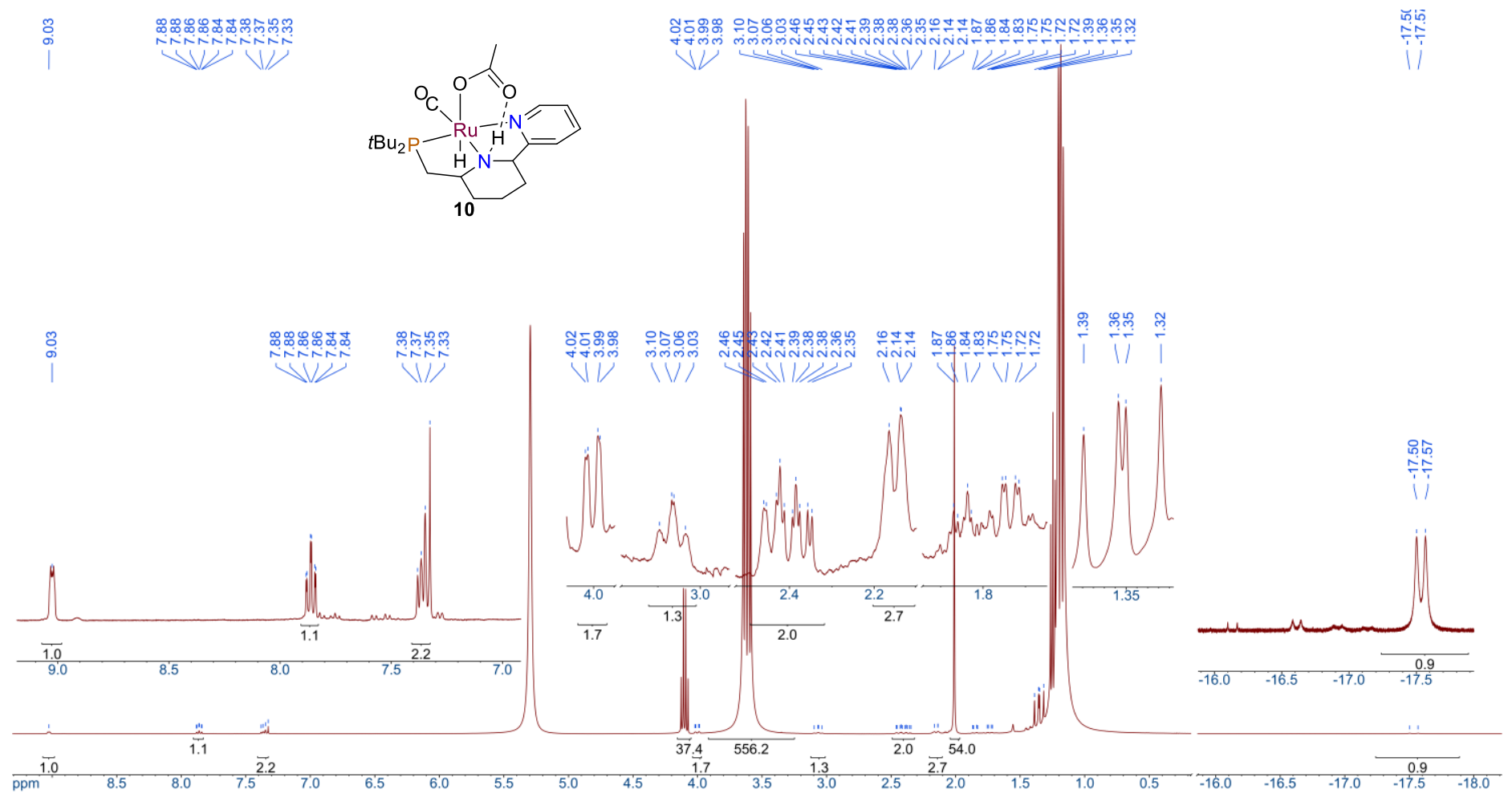

Figure S15. The ${ }^{1} \mathrm{H}$ NMR spectrum of the solution formed upon heating $20 \mathrm{mg}$ of 2 in $0.65 \mathrm{ml}$ of EtOH at $80{ }^{\circ} \mathrm{C}$ for $6 \mathrm{~h}$. This spectrum displays the resonances of ethyl acetate produced by dehydrogenative coupling of ethanol. A trace amount of benzene (present in $\mathbf{2}$ ) is seen at $7.33 \mathrm{ppm}$.



Figure S16. The ${ }^{13} \mathrm{C}\left\{{ }^{1} \mathrm{H}\right\}$ NMR spectrum of the solution formed upon heating $20 \mathrm{mg}$ of 2 in $0.65 \mathrm{ml} \mathrm{of} \mathrm{EtOH}$ at $80{ }^{\circ} \mathrm{C}$ for $6 \mathrm{~h}$. This spectrum also displays the resonances of ethyl acetate produced by dehydrogenative coupling of ethanol. A trace amount of benzene (present in 2) is seen at $129.3 \mathrm{ppm}$. 


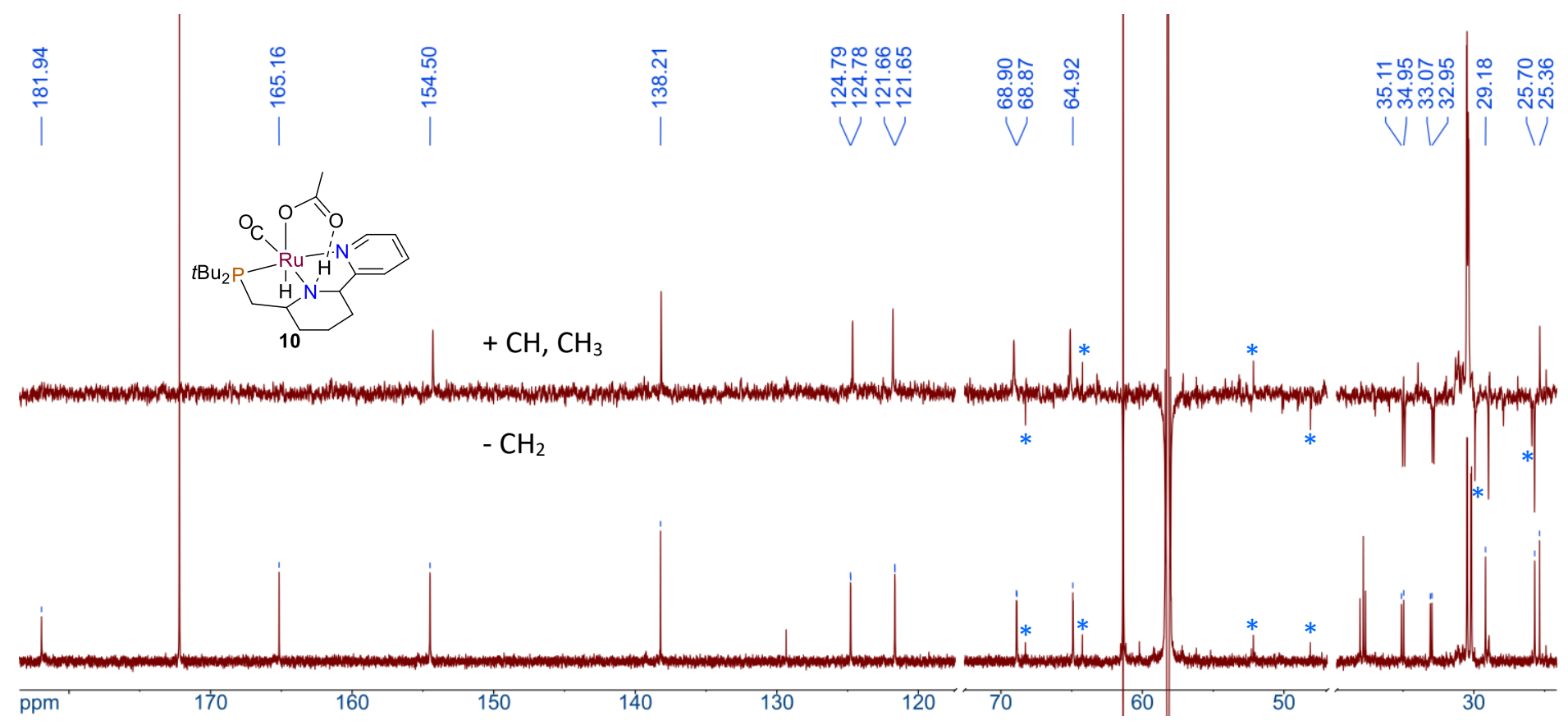

Figure S17. The ${ }^{13} \mathrm{C}\left\{{ }^{1} \mathrm{H}\right\}$ NMR overlaid by a DEPT-135 spectrum of the solution formed upon heating $20 \mathrm{mg}$ of 2 in $0.65 \mathrm{ml}$ of EtOH at $80^{\circ} \mathrm{C}$ for $6 \mathrm{~h}$. This spectrum also displays the resonances of ethyl acetate produced by dehydrogenative coupling of ethanol. The intense ethanol solvent peaks are responsible for a series of artefacts noted by the blue stars.

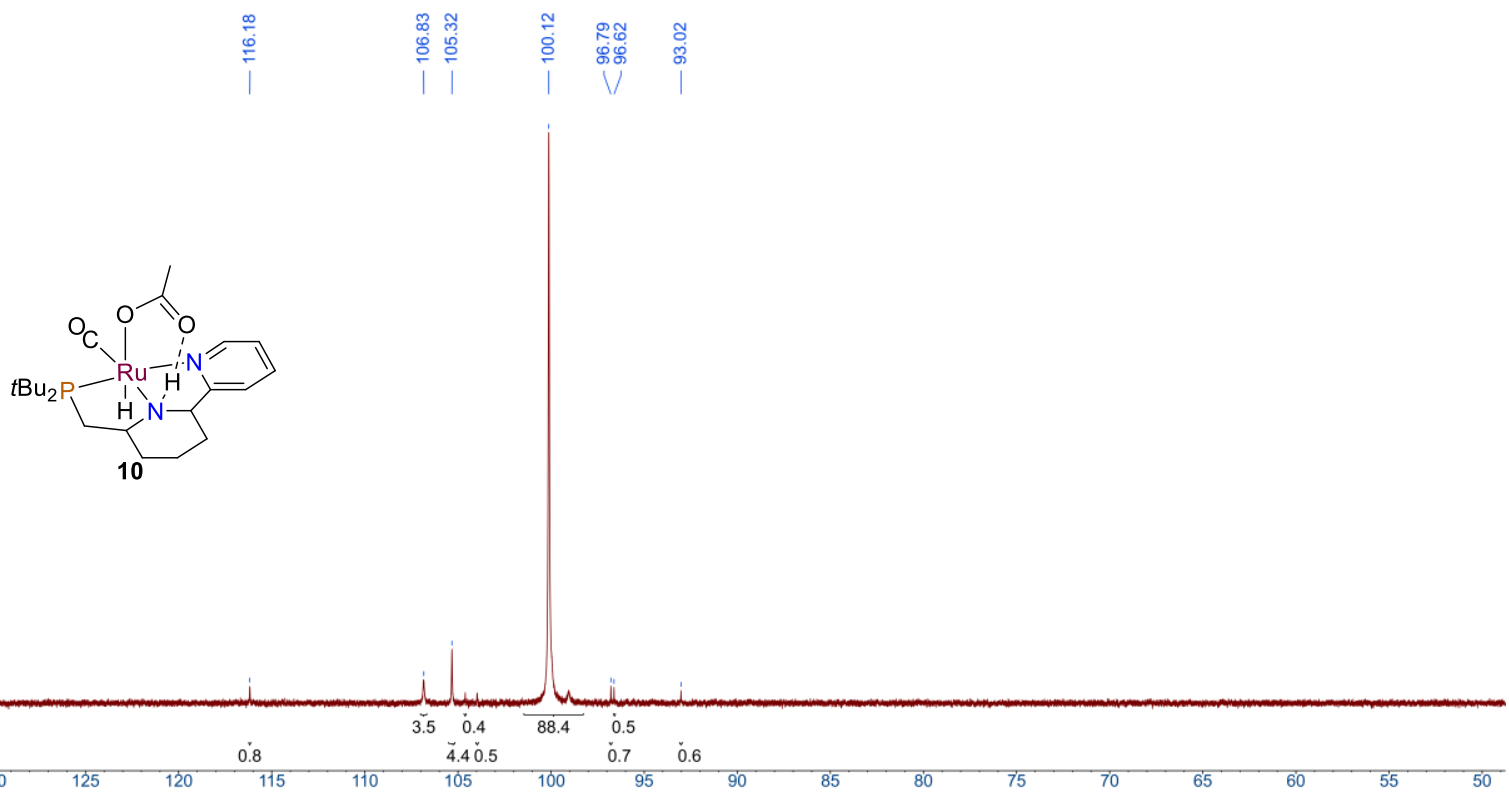

Figure S18. The ${ }^{31} \mathrm{P}\left\{{ }^{1} \mathrm{H}\right\}$ NMR spectrum of the solution formed upon heating $20 \mathrm{mg}$ of 2 in $0.65 \mathrm{ml}$ of EtOH at $80{ }^{\circ} \mathrm{C}$ for $6 \mathrm{~h}$. 


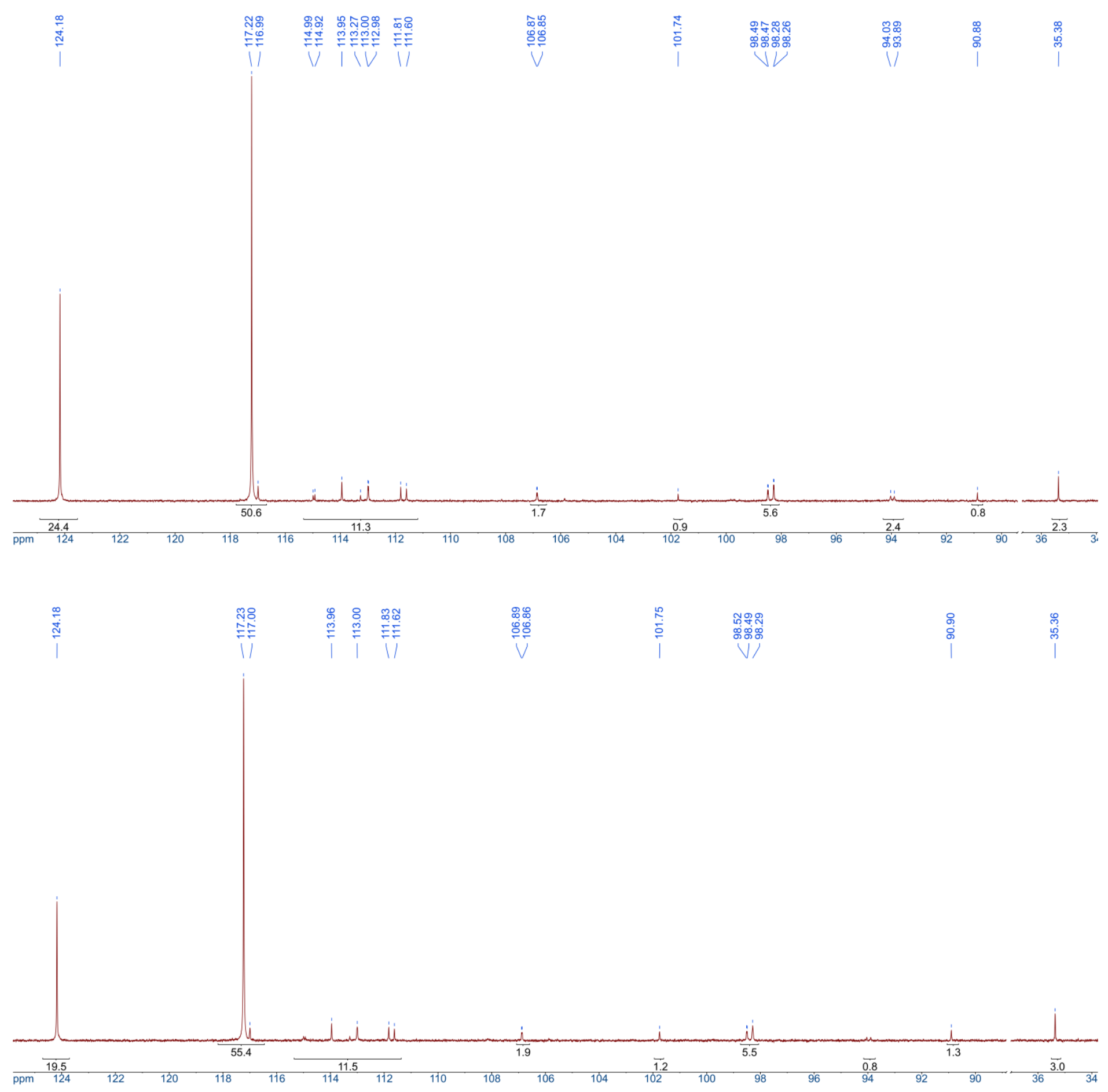

Figure S19. The ${ }^{31} \mathrm{P}\left\{{ }^{1} \mathrm{H}\right\}$ NMR spectra of the solutions formed after heating 3 in benzene under 50 bar $\mathrm{H}_{2}$, at $100{ }^{\circ} \mathrm{C}$ for $2 \mathrm{~h}$ (top spectrum) and $4 \mathrm{~h}$. The resonance at $124.2 \mathrm{ppm}$ is due to 3 . 




Figure S20. The ${ }^{1} \mathrm{H}$ NMR spectrum of the material formed after heating 3 in benzene under 50 bar $\mathrm{H}_{2}$, at $100{ }^{\circ} \mathrm{C}$ for $2 \mathrm{~h}$. The product solution was evaporated and extracted in hexane. The hexane extract was filtered and evaporated, and the residue was redissolved in $\mathrm{C}_{6} \mathrm{D}_{6}$. Some residual hexane is seen in the proton spectrum. The hydride resonances at -26.57 and -4.18 are due to 1 and 3 , respectively.

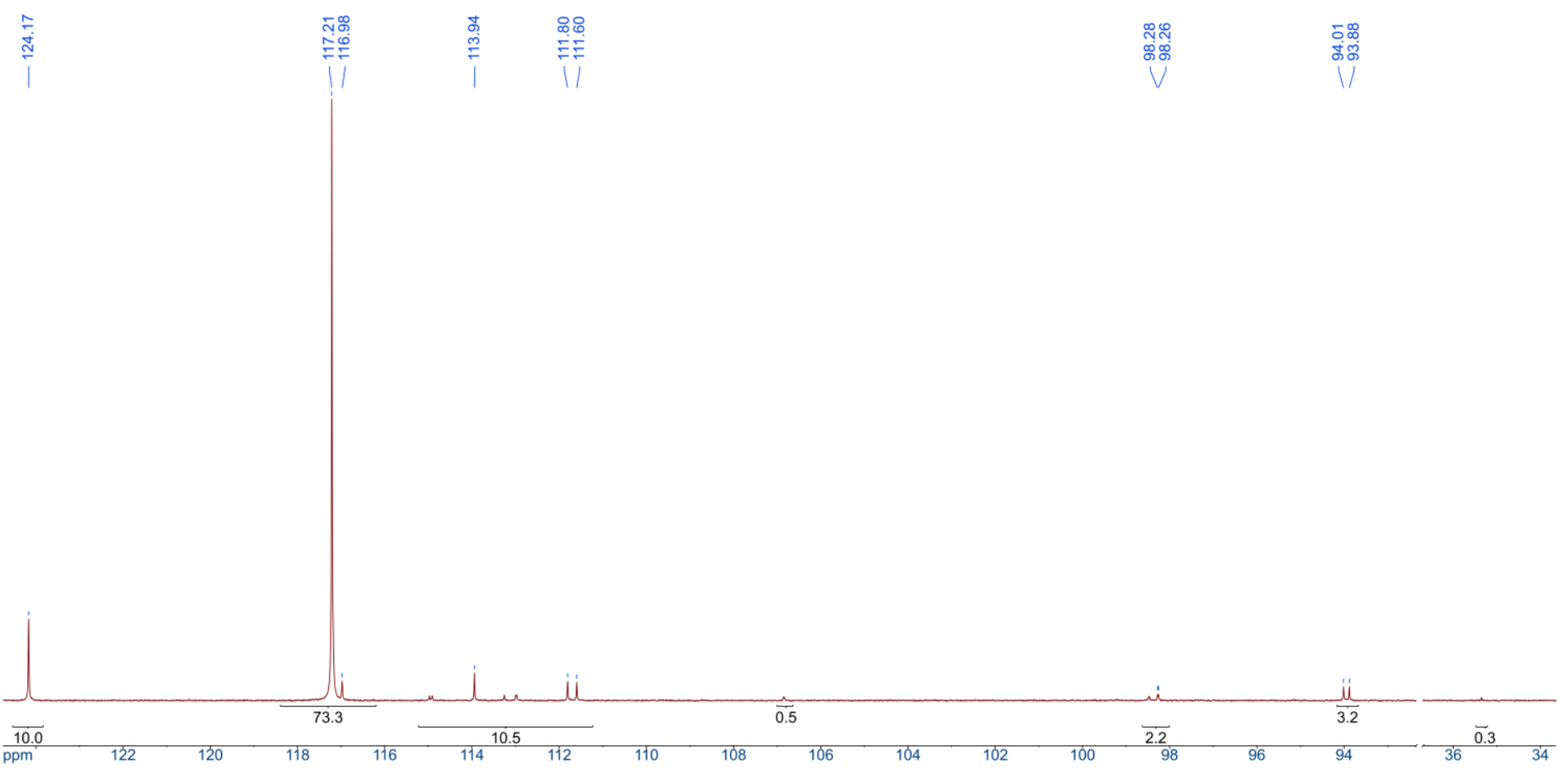

Figure S21. The ${ }^{31} \mathrm{P}\left\{{ }^{1} \mathrm{H}\right\}$ NMR spectrum of the material formed upon heating 3 in benzene under 50 bar $\mathrm{H}_{2}$, at $100{ }^{\circ} \mathrm{C}$ for $2 \mathrm{~h}$. The product solution was evaporated and extracted in hexane. The hexane extract was filtered and evaporated, and the residue was redissolved in $\mathrm{C}_{6} \mathrm{D}_{6}$. 




Figure S22. The ${ }^{1} \mathrm{H}$ NMR spectrum of 7 formed by treating 20 mg of RuHCl(CO)[PNN] (11) with ca. 1.5 equiv of Li[HBEt ${ }_{3}$ ] (1M solution in THF) in $0.7 \mathrm{~mL}$ of THF-d 8 in a J. Young NMR tube. The product solution was saturated with $\mathrm{H}_{2}$ and sealed under an $\mathrm{H}_{2}$ atmosphere. The spectrum was collected at $-30^{\circ} \mathrm{C}$.

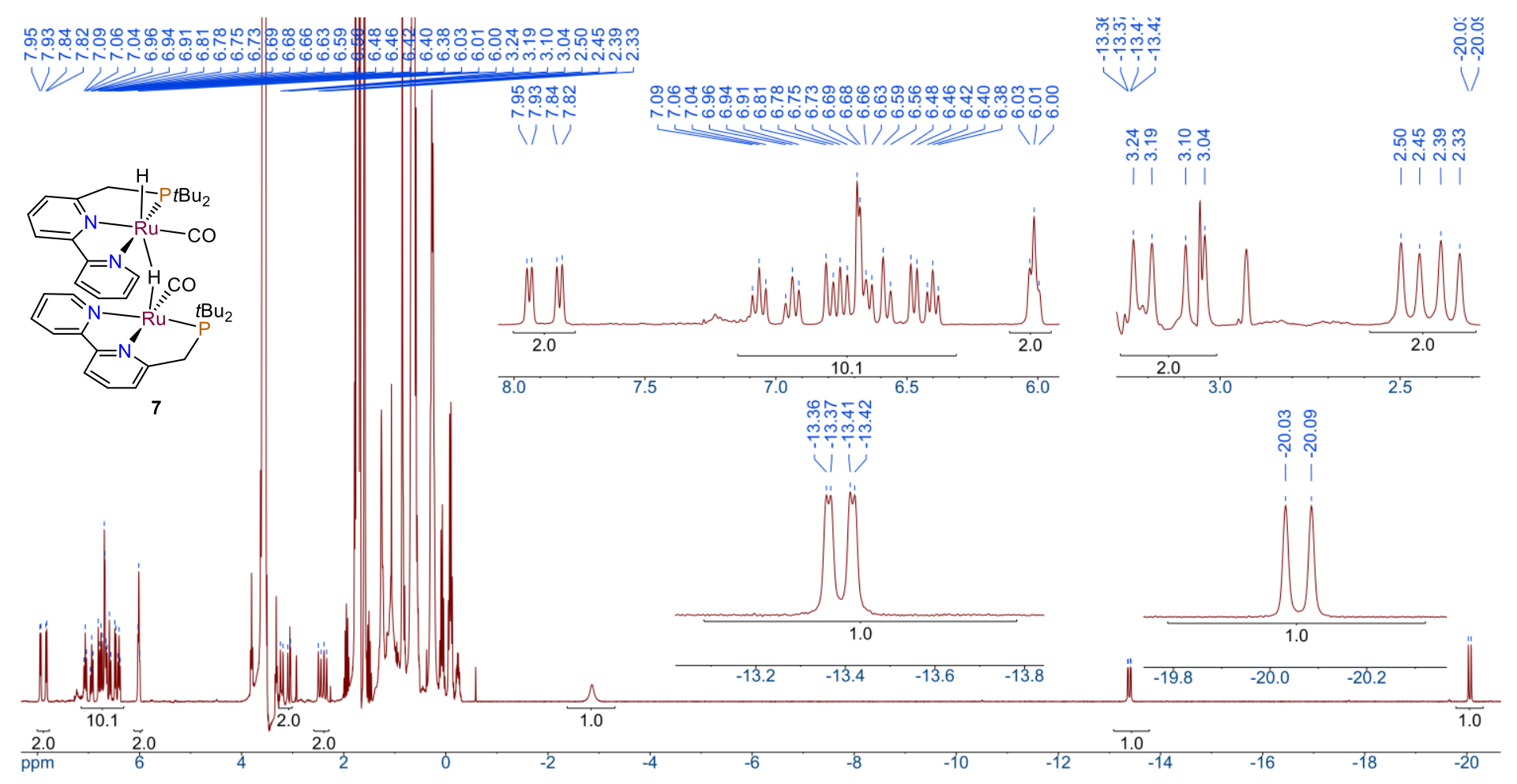


solution in THF) in $0.7 \mathrm{~mL}$ of THF-d 8 in a J. Young NMR tube. The product solution was saturated with $\mathrm{H}_{2}$ and sealed under an $\mathrm{H}_{2}$ atmosphere. The spectrum was collected at $-30{ }^{\circ} \mathrm{C}$. 


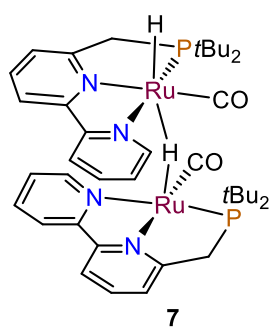



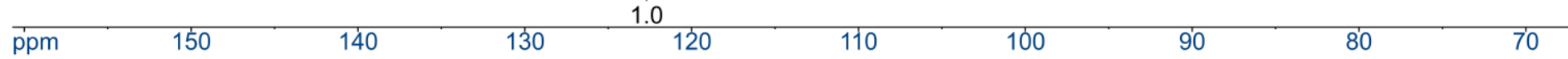

Figure S24. The ${ }^{31} \mathrm{P}\left\{{ }^{1} \mathrm{H}\right\}$ NMR spectrum of 7 formed by treating $20 \mathrm{mg}$ of RuHCI(CO)[PNN] (11) with ca. 1.5 equiv of Li[HBEt $\left.{ }_{3}\right](1 \mathrm{M}$ solution in THF) in $0.7 \mathrm{~mL}$ of THF-d $\mathrm{d}_{8}$ in a J. Young NMR tube. The product solution was saturated with $\mathrm{H}_{2}$ and sealed under an $\mathrm{H}_{2}$ atmosphere. The spectrum was collected at $-30^{\circ} \mathrm{C}$.


\begin{tabular}{lllllllll}
\hline $\mathrm{ppm}$ & 150 & 140 & 130 & 1.0 & 120 & 110 & 100 & 90
\end{tabular}

Figure S25. The ${ }^{31} \mathrm{P}\left\{{ }^{1} \mathrm{H}\right\}$ NMR spectrum of 7 formed by reacting $20 \mathrm{mg}$ of RuH(CO)[PNN] (2) with 1 atm $\mathrm{H}_{2}$ in $0.7 \mathrm{~mL}$ of ethyl acetate in a J. Young NMR tube while maintaining the temperature of the tube between -30 and $-50{ }^{\circ} \mathrm{C}$. The product solution was saturated with $\mathrm{H}_{2}$ and sealed under an $\mathrm{H}_{2}$ atmosphere. The spectrum was collected at $-30{ }^{\circ} \mathrm{C}$. 


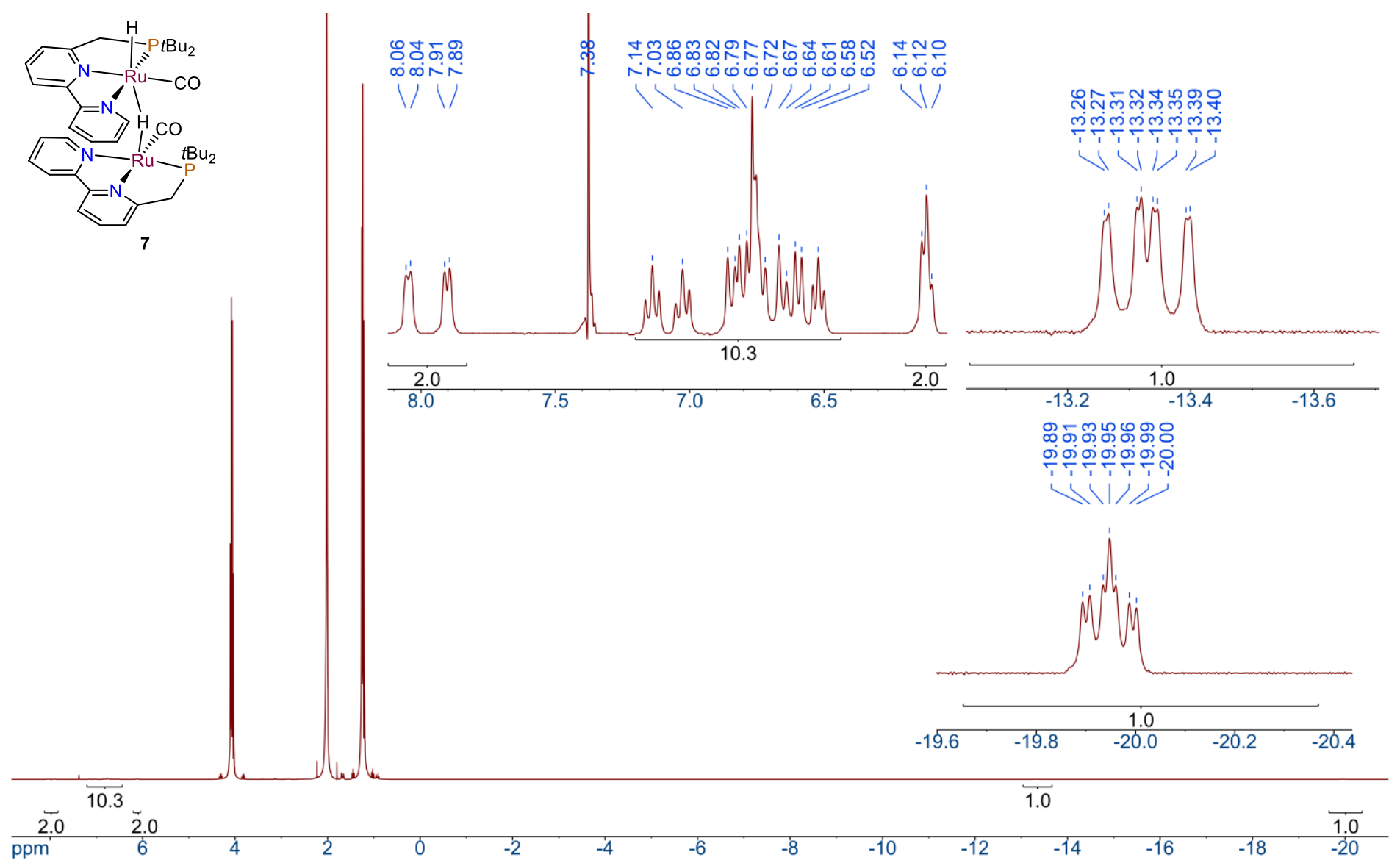

Figure S26. The ${ }^{1} \mathrm{H}$ NMR spectrum of 7 formed by reacting $20 \mathrm{mg}$ of $\mathrm{RuH}(\mathrm{CO})[\mathrm{PNN}](2)$ with 1 atm $\mathrm{H}_{2}$ in $0.7 \mathrm{~mL}$ of ethyl acetate in a J. Young NMR tube while maintaining the temperature of the tube between -30 and $-50{ }^{\circ} \mathrm{C}$. The product solution was saturated with $\mathrm{H}_{2}$ and sealed under an $\mathrm{H}_{2}$ atmosphere. The spectrum was collected at $-30^{\circ} \mathrm{C}$. The peak at 7.38 ppm is due to the residual $\mathrm{C}_{6} \mathrm{H}_{6}$ in 2 .
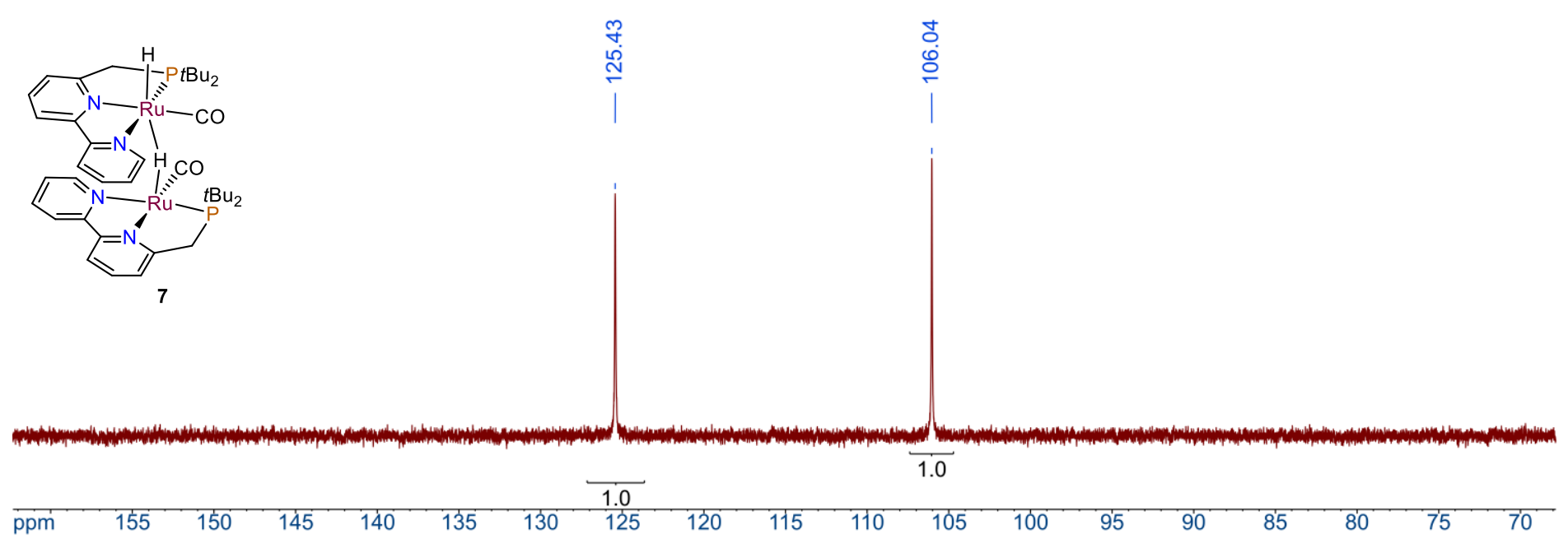

Figure S27. The ${ }^{31} \mathrm{P}\left\{{ }^{1} \mathrm{H}\right\}$ NMR spectrum of 7 formed by reacting $20 \mathrm{mg}$ of $\mathrm{RuH}(\mathrm{CO})[\mathrm{PNN}](2)$ with 1 atm $\mathrm{H}_{2}$ in $0.7 \mathrm{~mL}$ of methylcyclohexane- $\mathrm{d}_{14}$ in a J. Young NMR tube. The product solution was saturated with $\mathrm{H}_{2}$ and sealed under an $\mathrm{H}_{2}$ atmosphere. The spectrum was collected at $-30^{\circ} \mathrm{C}$. 


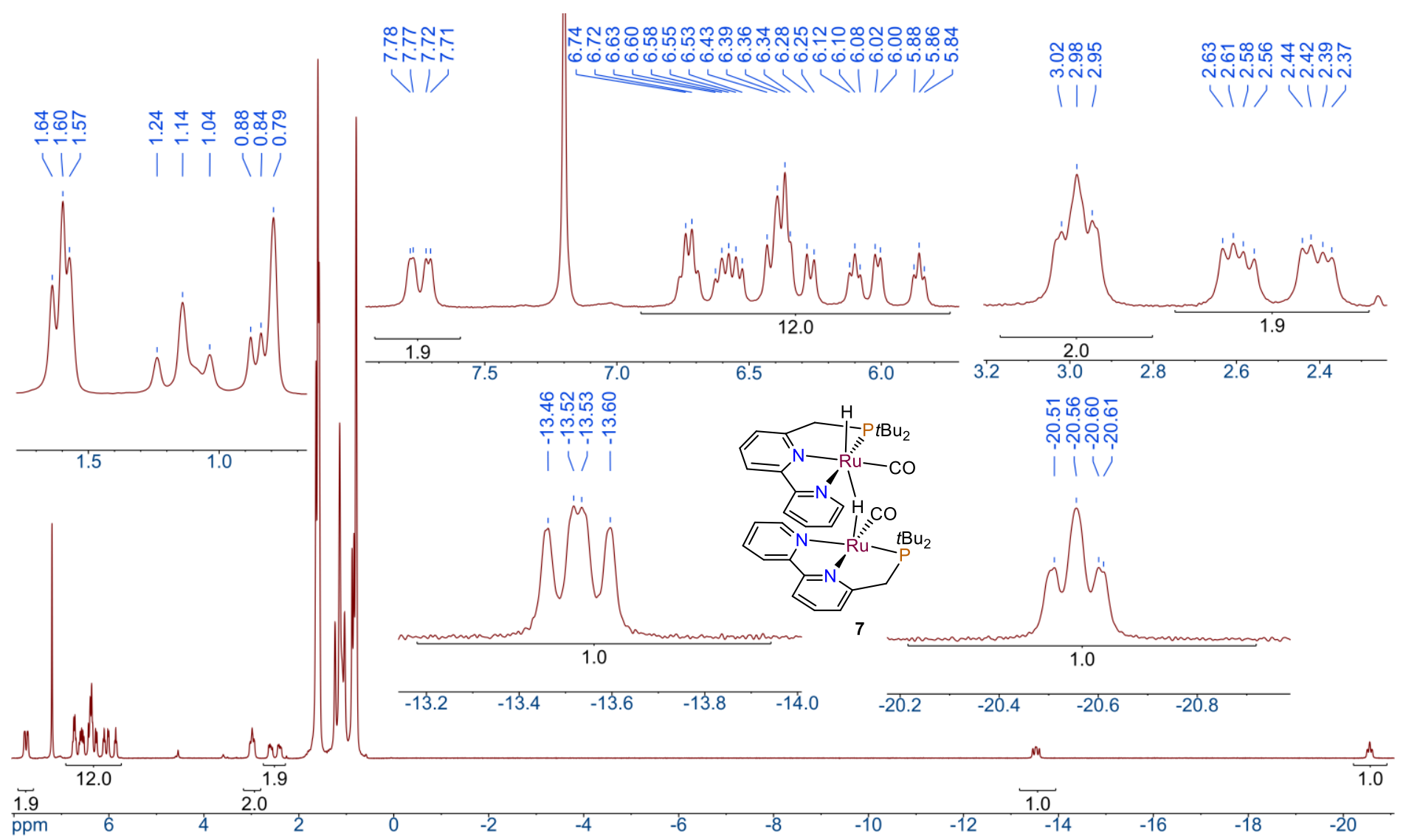

Figure S28. The ${ }^{1} \mathrm{H}$ NMR spectrum of 7 formed by reacting $20 \mathrm{mg}$ of $\mathrm{RuH}(\mathrm{CO})$ [PNN] (2) with 1 atm $\mathrm{H}_{2}$ in $0.7 \mathrm{~mL}$ of methylcyclohexane$\mathrm{d}_{14}$ in a J. Young NMR tube. The product solution was saturated with $\mathrm{H}_{2}$ and sealed under an $\mathrm{H}_{2}$ atmosphere. The spectrum was collected at $-30^{\circ} \mathrm{C}$. The peak at $7.20 \mathrm{ppm}$ is due to the residual $\mathrm{C}_{6} \mathrm{H}_{6}$ in $\mathbf{2}$.

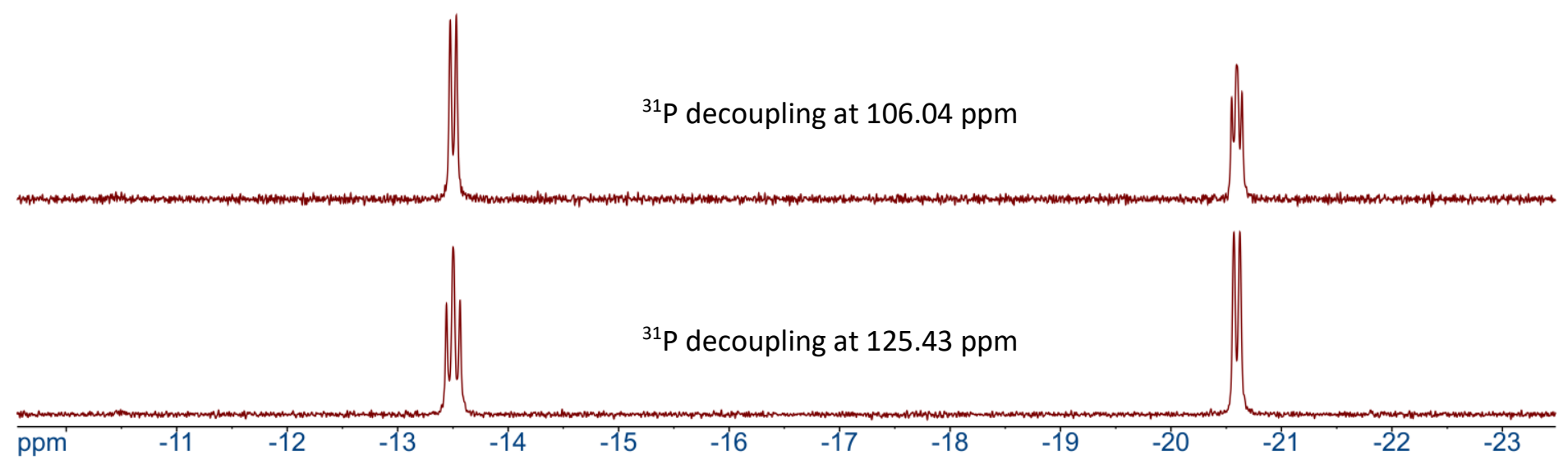

Figure S29. The hydride region of selectively ${ }^{31} \mathrm{P}$-decoupled ${ }^{1} \mathrm{H}$ NMR spectra of 7 formed by reacting $20 \mathrm{mg}$ of RuH(CO)[PNN] (2) with 1 atm $\mathrm{H}_{2}$ in $0.7 \mathrm{~mL}$ of methylcyclohexane- $\mathrm{d}_{14}$ in a J. Young NMR tube. The product solution was saturated with $\mathrm{H}_{2}$ and sealed under an $\mathrm{H}_{2}$ atmosphere. The spectrum was collected at $-50^{\circ} \mathrm{C}$. 

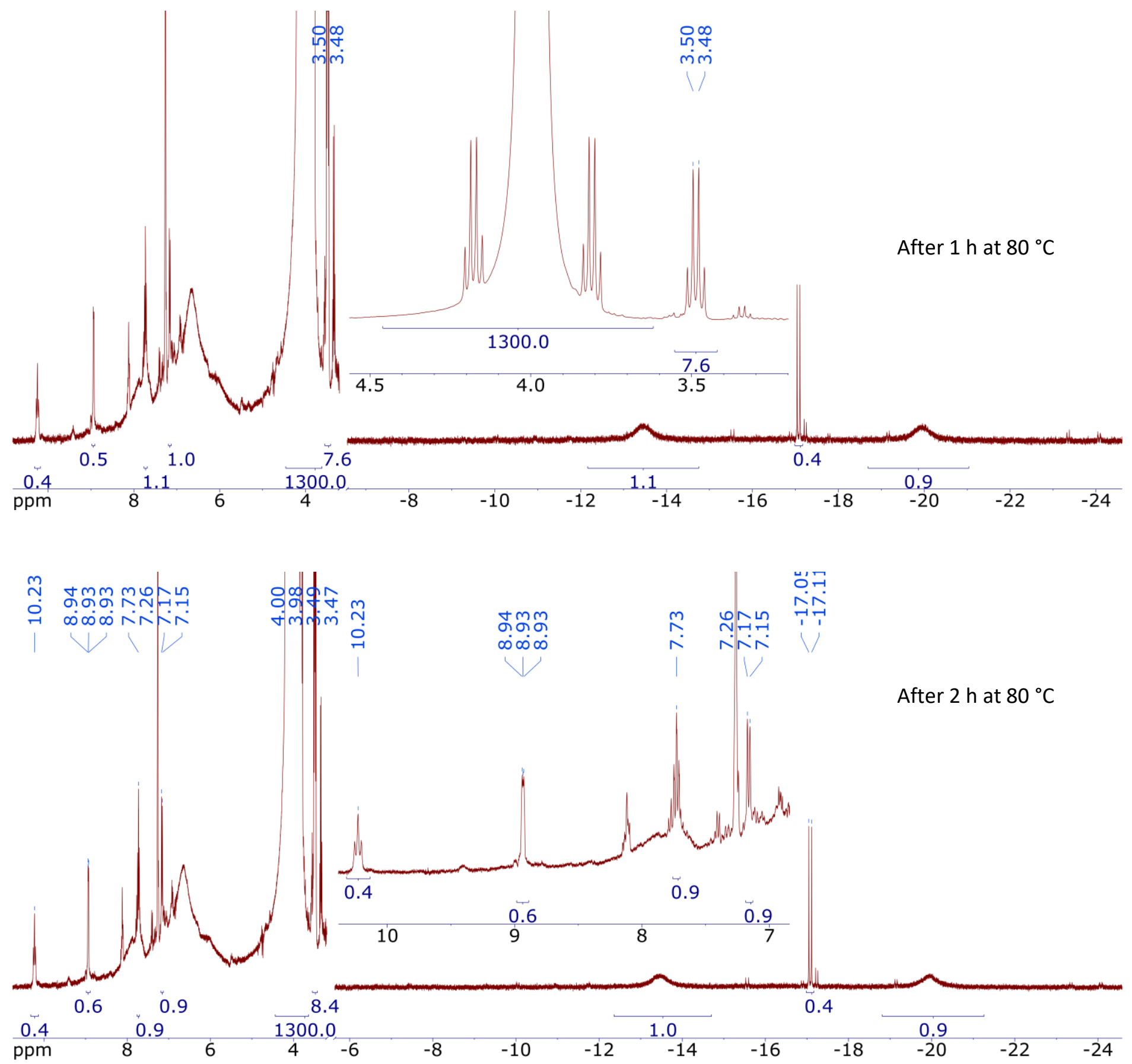

Figure S30. ${ }^{1} \mathrm{H}$ NMR spectra of a solution of 7 in ethyl acetate, formed by reacting $20 \mathrm{mg}$ of RuH(CO)[PNN] (2) with 1 atm $\mathrm{H}_{2}$ in a J. Young NMR tube, followed by heating at $80^{\circ} \mathrm{C}$. Complex 7 is observed as the two very broad resonances near -13.5 and -20 ppm. The corresponding aromatic proton resonances are seen as a broad "hump" between 6 and 8 ppm. The spectra show a peak of $\mathrm{C}_{6} \mathrm{H}_{6}$ at $7.26 \mathrm{ppm}$ (benzene co-crystallized with 2). A new complex formed from 7 upon heating: it appears as a doublet at $-17.07\left({ }^{2} J(H-P)=\right.$ $26.3 \mathrm{~Hz}$ ). The pyridine resonances at $7.16(\mathrm{~d}), 7.73(\mathrm{t}) .8 .93(\mathrm{~m})$ are tentatively attributed to this new complex. A triplet at $10.23 \mathrm{ppm}$ $(\mathrm{J}=10 \mathrm{~Hz})$ is apparent as well, however, the structural assignment is not clear, e.g., this might be the $\mathrm{NH}$ resonance of a piperidine, displaying a three-bond coupling to the two $\mathrm{CH}$ groups. Formation of ethanol is evident from the observation of the quartet at 3.48 ppm, near the quartet resonance of ethyl acetate at $3.99 \mathrm{ppm}$. The EtOH/Ru ratio is low, ca. 2 - 3, after $2 \mathrm{~h}$. The new complex formed in this solution from 7 seems to possess a hydrogenated PNN ligand. The ${ }^{31} \mathrm{P}$ NMR resonance of the new complex is at 102.1 ppm. This and the hydride shift $(-17.07$ ppm) are reminiscent of the shifts of the hydrogen-bonded ethoxide 9 (98.5 ppm and -16.68 ppm, respectively, in ethanol) and those of the acetate 10 (100 ppm and -17.54 ppm, respectively, in ethanol). 
Computed M06-L/def2-QZVP energies in ethyl ethanoate solvent continuum.

\begin{tabular}{|l|l|l|l|l|}
\hline species & $i, \mathrm{~cm}^{-1}$ & $\mathrm{E}$ & $\mathrm{H}$ & $\mathrm{G}$ \\
\hline $\mathrm{H}_{2}$, 0 bar & & -1.173675 & -1.160425 & -1.171522 \\
\hline Acetaldehyde & & -153.889269 & -153.828940 & -153.858681 \\
\hline Ethanol & & -155.096095 & -155.010778 & -155.041431 \\
\hline Ethyl acetate & & -307.817904 & -307.691320 & -307.731785 \\
\hline 1-ethoxy ethanol & & -308.994872 & -308.845162 & -308.885503 \\
\hline TS9 & -1364.8 & -464.056147 & -463.826880 & -463.878828 \\
\hline
\end{tabular}

Computed M06-2X/def2-QZVP energies in ethyl ethanoate solvent continuum.

\begin{tabular}{|l|l|l|l|l|}
\hline species & $i, \mathrm{~cm}^{-1}$ & $\mathrm{E}$ & $\mathrm{H}$ & $\mathrm{G}$ \\
\hline $\mathrm{H}_{2}, 50$ bar & & -1.169509 & -1.155985 & -1.167071 \\
\hline Acetaldehyde & & -153.842008 & -153.781287 & -153.811024 \\
\hline Ethanol & & -155.049783 & -154.964172 & -154.994856 \\
\hline Ethyl acetate & & -307.730524 & -307.603286 & -307.643298 \\
\hline 1-ethoxy ethanol & & -308.912069 & -308.761480 & -308.801732 \\
\hline TS9 & -990.7 & -463.928646 & -463.697141 & -463.748119 \\
\hline
\end{tabular}

Computed M06-L energies in ethyl ethanoate solvent continuum. BS1 = def2-QZVP (Ru), def2-TZVP (H, C, $\mathrm{N}, \mathrm{O}, \mathrm{P})$.

\begin{tabular}{|c|c|c|c|c|c|}
\hline species & $i, \mathrm{~cm}^{-1}$ & $\mathrm{E}(\mathrm{BS} 1)$ & E(def2-QZVP) & $\mathrm{H}(\mathrm{BS} 1)$ & G(BS1) \\
\hline Complex 5a & & -1403.430538 & -1403.525232 & -1402.902684 & -1402.984472 \\
\hline Complex 8 & & -1404.623098 & -1404.719928 & -1404.07435 & -1404.155793 \\
\hline Complex 8·EtOH & & -1559.719846 & -1559.830493 & -1559.08343 & -1559.177810 \\
\hline Complex $9 \cdot \mathrm{EtOH}$, structure 1 & & -1713.636393 & -1713.757895 & -1712.932294 & -1713.035141 \\
\hline Complex $9 \cdot \mathrm{EtOH}$, structure 2 & & -1713.635699 & -1713.757190 & -1712.931828 & -1713.035635 \\
\hline TS1 & -651.0 & -1867.503463 & -1867.636958 & -1866.740390 & -1866.851276 \\
\hline Int 1 & & -1867.510700 & -1867.644164 & -1866.742805 & -1866.855187 \\
\hline TS2 & -53.1 & -1867.501560 & -1867.635200 & -1866.733853 & -1866.845427 \\
\hline Int 2 & & -1867.509575 & -1867.643325 & -1866.740019 & -1866.851207 \\
\hline TS3 & -143.1 & -1867.505395 & -1867.639037 & -1866.73767 & -1866.849127 \\
\hline TS4 & -133.2 & -1713.611322 & -1713.733003 & -1712.909810 & -1713.014306 \\
\hline Int 3 & & -1713.620697 & -1713.742327 & -1712.91795 & -1713.021332 \\
\hline TS5 & -73.1 & -1714.788728 & -1714.911959 & -1714.072677 & -1714.182942 \\
\hline Int 4 & & -1714.796979 & -1714.919948 & -1714.076795 & -1714.184026 \\
\hline TS6 & -267.8 & -1714.796521 & -1714.919262 & -1714.076710 & -1714.183585 \\
\hline TS7 & -443.7 & -1713.601171 & -1713.723401 & -1712.903305 & -1713.007446 \\
\hline TS8 & -87.5 & -1867.505110 & -1867.638455 & -1866.738329 & -1866.850491 \\
\hline Int 5 & & -1867.526413 & -1867.659873 & -1866.757255 & -1866.867437 \\
\hline TS10 & -1464.7 & -1404.581207 & -1404.677221 & -1404.038858 & -1404.120345 \\
\hline Int 6 & & -1404.600298 & -1404.696378 & -1404.054816 & -1404.137065 \\
\hline Int 7 & & -1559.698670 & -1559.808397 & -1559.066429 & -1559.163602 \\
\hline TS11 & -1377.5 & -1559.689053 & -1559.798155 & -1559.061552 & -1559.155580 \\
\hline Int 8 & & -1558.523334 & -1558.631610 & -1557.907904 & -1558.000608 \\
\hline TS12 & -880.6 & -1558.517961 & -1558.625495 & -1557.907040 & -1557.997735 \\
\hline
\end{tabular}


Computed M06-L energies in benzene solvent continuum. BS1 = def2-QZVP $(R u)$, def2-TZVP $(H, C, N, O$, P).

\begin{tabular}{|c|c|c|c|c|}
\hline species & $\mathrm{E}(\mathrm{BS} 1)$ & E(def2-QZVP) & $\mathrm{H}(\mathrm{BS} 1)$ & $\mathrm{G}(\mathrm{BS} 1)$ \\
\hline Complex 2 & -1399.815901 & -1399.905421 & -1399.359031 & -1399.439428 \\
\hline Complex 4 & -1401.013250 & -1401.104155 & -1400.537172 & -1400.617276 \\
\hline Complex 5a & -1403.427901 & -1403.522491 & -1402.899571 & -1402.982271 \\
\hline Complex 6 & -2806.888046 & -2807.075387 & -2805.831566 & -2805.970939 \\
\hline Complex 7 & -2800.879042 & -2801.059057 & -2799.941335 & -2800.077759 \\
\hline Complex 8 & -1404.615547 & -1404.712327 & -1404.066471 & -1404.148078 \\
\hline Complex 8·EtOH & -1559.714720 & -1559.825226 & -1559.077968 & -1559.173130 \\
\hline Complex 9 & -1558.529035 & -1558.636951 & -1557.91181 & -1558.003398 \\
\hline Complex 9·EtOH & -1713.633053 & -1713.754405 & -1712.928360 & -1713.032184 \\
\hline $\mathrm{Ru}^{(0)}(\mathrm{CO})[\mathrm{PNN}]$ (singlet) & -1399.812793 & -1399.902785 & -1399.352524 & -1399.433424 \\
\hline $\mathrm{Ru}^{(0)}(\mathrm{CO})[\mathrm{PNN}]$ (triplet) & -1399.806101 & -1399.896009 & -1399.346855 & -1399.430247 \\
\hline TS from 2 to $\mathrm{Ru}^{(0)}(\mathrm{CO})[\mathrm{PNN}]$ (singlet) & -1399.754017 & -1399.843752 & -1399.299879 & -1399.380535 \\
\hline TS from 2 to $\mathrm{Ru}^{(0)}(\mathrm{CO})[\mathrm{PNN}]$ (triplet) & -1399.728285 & -1399.817900 & -1399.275406 & -1399.357064 \\
\hline 17-electron, aromatized $\mathrm{Ru}^{(\mathrm{l})} \mathrm{H}(\mathrm{CO})[\mathrm{PNN}]$ (doublet) & -1400.419318 & -1400.509763 & -1399.951107 & -1400.032715 \\
\hline EtOH, M06-L/def2-QZVP & & -155.095174 & -155.009719 & -155.040356 \\
\hline $\mathrm{H}_{2}$ gas (1 atm) M06-L/def2-QZVP & & -1.173675 & -1.160425 & -1.175216 \\
\hline
\end{tabular}


Typical g16 input file:

$\%$ chk=filename.chk

$\%$ mem=16GB

$\%$ NProcShared $=16$

\#opt freq rM06L/genecp/W06 scrf=(SMD, solvent=Benzene,read) Symmetry=None

comments

01

Coordinates of the atoms

HCNOPO

def2TZVP

$* * * *$

Ru 0

def2QZVP

$* * * *$

Ru 0

def2

NonSymmetricPCM

--Link1--

\%chk=filename.chk

$\%$ mem=16GB

$\%$ NProcShared $=16$

\#opt=readfc geom=check guess=read rM06L/genecp/W06 scrf=(SMD, solvent=Benzene,read) Symmetry=None

comments

01

H C N O P Ru 0

def2QZVP

$* * * *$

Ru 0

def2

NonSymmetricPCM 
Western Univeristy Sample Code b20094

X-ray Structure Report for complex 5, CCDC \#2008082

\author{
Prepared by \\ Louise N. Dawe, PhD \\ Department of Chemistry and Biochemistry \\ Wilfrid Laurier University \\ Science Building \\ 75 University Ave. W. \\ Waterloo, ON, ON \\ ldawe@wlu.ca
}

February 24, 2020

Introduction

Dr. Paul D. Boyle, Department of Chemistry X-Ray Facility, Western University, is acknowledged for assistance with X-ray data collection.

$\mathrm{H}$-atoms, except $\mathrm{H} 1$, were introduced in calculated positions and refined on a riding model. All other atoms were introduced in difference map positions and refined isotropically (H1) or anisotropically (all non-hydrogen atoms). 


\section{Experimental}

A single crystal of $\mathrm{C}_{20} \mathrm{H}_{33} \mathrm{~N}_{2} \mathrm{OPRu}$ was selected and collected on a Bruker APEX-II CCD diffractometer. The crystal was kept at 110(2) K during data collection. Using Olex2 [1], the structure was solved with the ShelXT [2] structure solution program using Direct Methods and refined with the ShelXL [3] refinement package using Least Squares minimization.

\section{Crystal structure determination}

Crystal Data for $\mathrm{C}_{20} \mathrm{H}_{33} \mathrm{~N}_{2} \mathrm{OPRu}(M=449.52 \mathrm{~g} / \mathrm{mol})$ : monoclinic, space group P2 $1 / \mathrm{c}$ (no. 14), $a=$ 17.503(4) $\AA, b=8.1902(19) \AA, c=15.888(4) \AA, \beta=113.789(13)^{\circ}, V=2084.1(9) \AA^{3}, Z=4, T=110(2) \mathrm{K}$, $\mu(\mathrm{MoK} \alpha)=0.839 \mathrm{~mm}^{-1}$, Dcalc $=1.433 \mathrm{~g} / \mathrm{cm}^{3}, 54853$ reflections measured $\left(5.136^{\circ} \leq 2 \theta \leq 54.206^{\circ}\right), 4606$ unique ( 3719 with $\left.\mathrm{I}>2 \sigma(\mathrm{I}) ; R_{\text {int }}=0.0524, \mathrm{R}_{\text {sigma }}=0.0281\right)$ which were used in all calculations. The final $R_{1}$ was $0.0264(\mathrm{I}>2 \sigma(\mathrm{I}))$ and $w R_{2}$ was 0.0589 (all data). 
Table 1 Crystal data and structure refinement

Identification code

Empirical formula

Formula weight

Temperature/K

Crystal system

Space group

$\mathrm{a} / \AA$

$\mathrm{b} / \AA$

$\mathrm{c} / \AA$

$\alpha /^{\circ}$

$\beta /{ }^{\circ}$

$\gamma /{ }^{\circ}$

Volume/ $/ \AA^{3}$

Z

$\rho_{\text {calc } g / \mathrm{cm}^{3}}$

$\mu / \mathrm{mm}^{-1}$

$\mathrm{F}(000)$

Crystal size $/ \mathrm{mm}^{3}$

Radiation b20094 (Western Sample Code)

$\mathrm{C}_{20} \mathrm{H}_{33} \mathrm{~N}_{2} \mathrm{OPRu}$

449.52

110(2)

monoclinic

$\mathrm{P} 2{ }_{1} / \mathrm{c}$

17.503(4)

8.1902(19)

$15.888(4)$

90

113.789(13)

90

2084.1(9)

4

1.433

0.839

936.0

$0.14 \times 0.122 \times 0.035$

$\operatorname{MoK} \alpha(\lambda=0.71073)$

$2 \Theta$ range for data collection $/{ }^{\circ} 5.136$ to 54.206

Index ranges

Reflections collected

Independent reflections

Data/restraints/parameters

Goodness-of-fit on $\mathrm{F}^{2}$

Final $R$ indexes $[\mathrm{I}>=2 \sigma(\mathrm{I})]$

Final $\mathrm{R}$ indexes [all data]

Largest diff. peak/hole / e $\AA^{-3} 0.45 /-0.61$

54853

1.024
$-22 \leq \mathrm{h} \leq 22,-10 \leq \mathrm{k} \leq 10,-20 \leq 1 \leq 20$

4606 [3719 with I $\left.>2 \sigma(I) ; R_{\text {int }}=0.0524, R_{\text {sigma }}=0.0281\right]$

$4606 / 0 / 236$

$\mathrm{R}_{1}=0.0264, \mathrm{wR}_{2}=0.0548$

$\mathrm{R}_{1}=0.0388, \mathrm{wR}_{2}=0.0589$ 
Table 2 Fractional Atomic Coordinates $\left(\times 10^{4}\right)$ and Equivalent Isotropic Displacement Parameters $\left(\AA^{2} \times 10^{3}\right)$. Ueq is defined as $1 / 3$ of of the trace of the orthogonalised UIJ tensor.

\begin{tabular}{|c|c|c|c|c|}
\hline Atom & $x$ & $y$ & $z$ & $\mathbf{U}(\mathbf{e q})$ \\
\hline Ru1 & $7065.0(2)$ & $7786.7(2)$ & $3097.0(2)$ & $19.31(6)$ \\
\hline $\mathrm{P} 1$ & $8182.9(3)$ & $6657.5(7)$ & $2935.3(4)$ & $20.66(13)$ \\
\hline $\mathrm{O} 1$ & $6740.7(15)$ & $10403(3)$ & $1670.2(15)$ & $64.8(7)$ \\
\hline N1 & $6043.6(11)$ & $8183(2)$ & $3457.0(12)$ & $22.7(4)$ \\
\hline $\mathrm{N} 2$ & $7056.3(11)$ & $5722(2)$ & $3734.0(12)$ & $22.8(4)$ \\
\hline C16 & $8102.8(14)$ & $6299(3)$ & $1727.9(16)$ & $26.8(5)$ \\
\hline C14 & $9976.5(14)$ & $6642(3)$ & $3541.5(17)$ & $32.9(6)$ \\
\hline C12 & $9252.1(14)$ & 7491 (3) & $3673.4(16)$ & $26.9(5)$ \\
\hline $\mathrm{C} 8$ & $6858.3(16)$ & $2688(3)$ & $4581.5(18)$ & $35.8(6)$ \\
\hline C10 & $7721.7(14)$ & $4490(3)$ & $4019.2(16)$ & $26.1(5)$ \\
\hline C18 & $8344.9(15)$ & $7852(3)$ & $1352.4(16)$ & $31.4(5)$ \\
\hline $\mathrm{C} 1$ & $5519.8(14)$ & $9478(3)$ & $3220.2(16)$ & $28.2(5)$ \\
\hline C9 & $7383.0(17)$ & $2778(3)$ & $4012.9(18)$ & $34.8(6)$ \\
\hline $\mathrm{C} 2$ & $4871.4(15)$ & $9652(3)$ & $3495.5(18)$ & $36.2(6)$ \\
\hline $\mathrm{C} 20$ & $6873.5(16)$ & $9406(3)$ & $2238.0(18)$ & $34.9(6)$ \\
\hline $\mathrm{C} 3$ & $4751.5(16)$ & $8452(4)$ & $4036(2)$ & $45.0(7)$ \\
\hline C13 & $9366.7(15)$ & $7235(3)$ & $4676.1(16)$ & $34.9(6)$ \\
\hline $\mathrm{C} 4$ & $5283.3(16)$ & $7130(4)$ & $4290.6(19)$ & $40.8(7)$ \\
\hline C15 & $9278.5(15)$ & $9334(3)$ & $3516.1(18)$ & $32.9(6)$ \\
\hline $\mathrm{C} 5$ & $5926.5(14)$ & $7005(3)$ & $3988.8(16)$ & $27.6(5)$ \\
\hline $\mathrm{C} 17$ & $7177.2(15)$ & $5932(3)$ & $1137.4(16)$ & $33.1(6)$ \\
\hline C6 & $6546.8(14)$ & $5629(3)$ & $4274.4(16)$ & $27.2(5)$ \\
\hline C19 & $8614.4(16)$ & $4854(3)$ & $1625.8(17)$ & $34.9(6)$ \\
\hline $\mathrm{C} 7$ & $6164.7(15)$ & 3951 (3) & $4235.8(18)$ & $34.5(6)$ \\
\hline C11 & $8167.6(14)$ & $4572(3)$ & $3362.3(17)$ & $26.8(5)$ \\
\hline
\end{tabular}


Table 3 Anisotropic Displacement Parameters $\left(\AA^{2} \times 10^{3}\right)$. The Anisotropic displacement factor exponent takes the form: $-2 \pi^{2}\left[h^{2} a^{* 2} U_{11}+2 h k a * b^{*} U_{12}+\ldots\right]$.

\begin{tabular}{|c|c|c|c|c|c|c|}
\hline Atom & $\mathbf{U}_{11}$ & $\mathbf{U}_{22}$ & $\mathbf{U}_{33}$ & $\mathbf{U}_{\mathbf{2 3}}$ & $\mathbf{U}_{13}$ & $\mathbf{U}_{12}$ \\
\hline $\mathrm{Ru} 1$ & $19.75(9)$ & $18.68(10)$ & $24.74(10)$ & $-0.36(8)$ & $14.43(7)$ & $0.71(8)$ \\
\hline $\mathrm{P} 1$ & $19.7(3)$ & $21.6(3)$ & $25.6(3)$ & $-1.3(2)$ & $14.4(2)$ & $0.7(2)$ \\
\hline $\mathrm{O} 1$ & $93.4(17)$ & $58.6(15)$ & $73.2(15)$ & $42.5(12)$ & $65.7(14)$ & $46.3(13)$ \\
\hline N1 & $18.0(9)$ & $27.4(11)$ & $24.7(10)$ & $-2.3(8)$ & $10.6(8)$ & $0.7(8)$ \\
\hline N2 & $22.8(10)$ & $22.3(10)$ & $29.4(10)$ & $2.0(8)$ & $16.9(8)$ & $0.6(8)$ \\
\hline C16 & $23.8(12)$ & $32.9(14)$ & $28.1(12)$ & $-5.8(10)$ & $14.9(10)$ & $1.3(10)$ \\
\hline C14 & $22.1(12)$ & $42.5(16)$ & $36.4(14)$ & $-1.0(12)$ & $14.0(11)$ & $1.2(11)$ \\
\hline C12 & $22.5(11)$ & $33.0(16)$ & $27.6(12)$ & $-4.1(10)$ & $12.7(10)$ & $-1.6(10)$ \\
\hline $\mathrm{C} 8$ & $40.7(15)$ & $31.8(15)$ & $36.6(14)$ & $8.4(11)$ & $17.4(12)$ & $-4.7(12)$ \\
\hline $\mathrm{C} 10$ & $26.0(12)$ & $23.9(13)$ & $28.9(12)$ & $0.5(10)$ & $11.6(10)$ & $1.8(10)$ \\
\hline C18 & $28.9(13)$ & $42.5(15)$ & $29.6(13)$ & $2.7(12)$ & $18.8(11)$ & $1.8(12)$ \\
\hline $\mathrm{C} 1$ & $24.4(12)$ & $33.1(14)$ & $29.1(13)$ & $-2.2(10)$ & $12.8(10)$ & $4.2(10)$ \\
\hline C9 & $40.6(15)$ & $25.0(13)$ & $40.7(14)$ & $2.8(12)$ & $18.3(12)$ & $-0.2(12)$ \\
\hline $\mathrm{C} 2$ & $22.7(12)$ & $47.6(17)$ & $40.3(15)$ & $-0.4(13)$ & $14.7(11)$ & $12.0(12)$ \\
\hline $\mathrm{C} 20$ & $41.9(15)$ & $35.8(15)$ & $40.6(15)$ & $6.8(12)$ & $30.7(13)$ & $15.3(12)$ \\
\hline $\mathrm{C} 3$ & $27.6(14)$ & $64(2)$ & $54.6(18)$ & $5.1(15)$ & $27.7(13)$ & $7.5(14)$ \\
\hline C13 & $28.3(13)$ & $46.4(16)$ & $30.1(13)$ & $-5.7(12)$ & $11.7(11)$ & $-6.2(12)$ \\
\hline $\mathrm{C} 4$ & $30.4(14)$ & $52.0(18)$ & $50.4(16)$ & $9.8(14)$ & $27.0(13)$ & $2.3(13)$ \\
\hline C15 & $27.8(13)$ & $35.3(15)$ & $41.8(15)$ & $-7.1(12)$ & $20.5(12)$ & $-9.5(11)$ \\
\hline $\mathrm{C} 5$ & $23.2(12)$ & $35.0(15)$ & $29.1(12)$ & $-0.7(11)$ & $15.1(10)$ & $-2.1(10)$ \\
\hline C17 & $29.4(13)$ & $41.5(16)$ & $30.4(13)$ & $-9.1(11)$ & $14.1(11)$ & $-3.6(11)$ \\
\hline C6 & $24.6(12)$ & $32.3(14)$ & $28.1(13)$ & $2.7(10)$ & $14.2(10)$ & $-2.0(10)$ \\
\hline C19 & $33.9(14)$ & $40.9(16)$ & $35.4(14)$ & $-12.1(12)$ & $19.8(12)$ & $4.3(12)$ \\
\hline $\mathrm{C} 7$ & $31.3(13)$ & $39.9(16)$ & $36.9(14)$ & $6.5(12)$ & $18.5(12)$ & $-6.2(12)$ \\
\hline $\mathrm{C} 11$ & $25.2(12)$ & $22.4(13)$ & $36.8(13)$ & $0.4(10)$ & $16.7(11)$ & $3.9(10$ \\
\hline
\end{tabular}




\section{Table 4 Bond Lengths}

\begin{tabular}{llrlll} 
Atom Atom & \multicolumn{1}{c}{ Length/A } & \multicolumn{2}{l}{ Atom Atom } & Length/A \\
Ru1 & P1 & $2.2706(7)$ & C16 & C19 & $1.532(3)$ \\
Ru1 & N1 & $2.1103(18)$ & C14 & C12 & $1.533(3)$ \\
Ru1 & N2 & $1.9740(19)$ & C12 & C13 & $1.538(3)$ \\
Ru1 & C20 & $1.835(3)$ & C12 & C15 & $1.533(3)$ \\
P1 & C16 & $1.889(2)$ & C8 & C9 & $1.528(3)$ \\
P1 & C12 & $1.890(2)$ & C8 & C7 & $1.519(4)$ \\
P1 & C11 & $1.842(2)$ & C10 & C9 & $1.521(3)$ \\
O1 & C20 & $1.169(3)$ & C10 & C11 & $1.536(3)$ \\
N1 & C1 & $1.352(3)$ & C1 & C2 & $1.379(3)$ \\
N1 & C5 & $1.351(3)$ & C2 & C3 & $1.376(4)$ \\
N2 & C10 & $1.467(3)$ & C3 & C4 & $1.378(4)$ \\
N2 & C6 & $1.468(3)$ & C4 & C5 & $1.394(3)$ \\
C16 & C18 & $1.535(3)$ & C5 & C6 & $1.503(3)$ \\
C16 & C17 & $1.539(3)$ & C6 & C7 & $1.519(3)$
\end{tabular}


Table 5 Bond Angles

\begin{tabular}{|c|c|c|c|c|c|c|c|}
\hline \multicolumn{3}{|c|}{ Atom Atom Atom } & \multirow{2}{*}{$\begin{array}{l}\text { Angle }^{\circ} \\
162.99(5)\end{array}$} & \multicolumn{3}{|c|}{ Atom Atom Atom } & \multirow{2}{*}{$\begin{array}{l}\text { Angle }^{\circ} \\
114.71(16)\end{array}$} \\
\hline N1 & Ru1 & $\mathrm{P} 1$ & & $\mathrm{C} 14$ & $\mathrm{C} 12$ & $\mathrm{P} 1$ & \\
\hline $\mathrm{N} 2$ & Ru1 & $\mathrm{P} 1$ & $83.93(5)$ & $\mathrm{C} 14$ & $\mathrm{C} 12$ & $\mathrm{C} 13$ & $108.1(2)$ \\
\hline N2 & Ru1 & N1 & $79.07(7)$ & $\mathrm{C} 14$ & $\mathrm{C} 12$ & $\mathrm{C} 15$ & $110.06(19)$ \\
\hline $\mathrm{C} 20$ & Ru1 & $\mathrm{P} 1$ & $96.75(7)$ & $\mathrm{C} 13$ & $\mathrm{C} 12$ & $\mathrm{P} 1$ & $106.04(15)$ \\
\hline $\mathrm{C} 20$ & Ru1 & N1 & $99.78(9)$ & $\mathrm{C} 15$ & $\mathrm{C} 12$ & $\mathrm{P} 1$ & $109.98(16)$ \\
\hline $\mathrm{C} 20$ & Ru1 & $\mathrm{N} 2$ & $164.57(10)$ & $\mathrm{C} 15$ & $\mathrm{C} 12$ & $\mathrm{C} 13$ & $107.6(2)$ \\
\hline $\mathrm{C} 16$ & $\mathrm{P} 1$ & Ru1 & $117.64(7)$ & $\mathrm{C} 7$ & $\mathrm{C} 8$ & C9 & $109.7(2)$ \\
\hline C16 & $\mathrm{P} 1$ & $\mathrm{C} 12$ & $109.60(10)$ & $\mathrm{N} 2$ & $\mathrm{C} 10$ & C9 & $111.91(19)$ \\
\hline C12 & $\mathrm{P} 1$ & Ru1 & $117.74(7)$ & $\mathrm{N} 2$ & $\mathrm{C} 10$ & $\mathrm{C} 11$ & $108.53(18)$ \\
\hline $\mathrm{C} 11$ & $\mathrm{P} 1$ & Ru1 & $101.24(7)$ & C9 & $\mathrm{C} 10$ & $\mathrm{C} 11$ & $109.80(19)$ \\
\hline $\mathrm{C} 11$ & $\mathrm{P} 1$ & $\mathrm{C} 16$ & $102.92(11)$ & N1 & $\mathrm{C} 1$ & $\mathrm{C} 2$ & $123.1(2)$ \\
\hline $\mathrm{C} 11$ & $\mathrm{P} 1$ & $\mathrm{C} 12$ & $105.32(11)$ & $\mathrm{C} 10$ & C9 & $\mathrm{C} 8$ & $111.5(2)$ \\
\hline $\mathrm{C} 1$ & N1 & Ru1 & $126.78(15)$ & C3 & $\mathrm{C} 2$ & $\mathrm{C} 1$ & $118.5(2)$ \\
\hline C5 & $\mathrm{N} 1$ & Ru1 & $114.73(15)$ & O1 & $\mathrm{C} 20$ & Ru1 & $178.0(3)$ \\
\hline $\mathrm{C} 5$ & N1 & $\mathrm{C} 1$ & $118.49(19)$ & $\mathrm{C} 2$ & C3 & $\mathrm{C} 4$ & $119.3(2)$ \\
\hline $\mathrm{C} 10$ & $\mathrm{~N} 2$ & Ru1 & $125.31(14)$ & $\mathrm{C} 3$ & $\mathrm{C} 4$ & C5 & $120.0(3)$ \\
\hline $\mathrm{C} 10$ & $\mathrm{~N} 2$ & C6 & $112.17(17)$ & N1 & $\mathrm{C} 5$ & $\mathrm{C} 4$ & $120.7(2)$ \\
\hline C6 & $\mathrm{N} 2$ & Ru1 & $118.43(14)$ & N1 & $\mathrm{C} 5$ & C6 & $116.81(19)$ \\
\hline C18 & C16 & $\mathrm{P} 1$ & $110.29(16)$ & $\mathrm{C} 4$ & $\mathrm{C} 5$ & C6 & $122.4(2)$ \\
\hline C18 & $\mathrm{C} 16$ & $\mathrm{C} 17$ & $108.2(2)$ & $\mathrm{N} 2$ & C6 & C5 & $108.66(18)$ \\
\hline $\mathrm{C} 17$ & $\mathrm{C} 16$ & $\mathrm{P} 1$ & $106.14(15)$ & $\mathrm{N} 2$ & C6 & $\mathrm{C} 7$ & $112.6(2)$ \\
\hline C19 & $\mathrm{C} 16$ & $\mathrm{P} 1$ & $114.87(17)$ & C5 & C6 & $\mathrm{C} 7$ & $114.5(2)$ \\
\hline C19 & $\mathrm{C} 16$ & $\mathrm{C} 18$ & $109.53(19)$ & $\mathrm{C} 8$ & $\mathrm{C} 7$ & C6 & $109.1(2)$ \\
\hline C19 & $\mathrm{C} 16$ & $\mathrm{C} 17$ & $107.5(2)$ & $\mathrm{C} 10$ & $\mathrm{C} 11$ & $\mathrm{P} 1$ & $112.15(16)$ \\
\hline
\end{tabular}


Table 6 Torsion Angles

$\begin{array}{llllllllll}\text { A } & \mathbf{B} & \mathbf{C} & \mathbf{D} & \text { Angle }^{\circ} & \text { A } & \mathbf{B} & \mathbf{C} & \mathbf{D} & \text { Angle }^{\circ}\end{array}$

Ru1P1 C16C18 85.25(16) C12P1 C16C19 $71.5(2)$

Ru1P1 C16C17 -31.75(19) C12P1 C11C10 $99.12(18)$

Ru1P1 C16C19 $150.40(15)$ C10N2 C6 C5 $175.72(18)$

Ru1P1 C12C14 178.67(14) C10N2 C6 C7 $\quad-56.4(3)$

Ru1P1 C12C13 59.46(18) C1 N1 C5 C4 $-0.4(3)$

Ru1P1 C12C15 -56.64(17) C1 N1 C5 C6 -177.1(2)

Ru1P1 C11C10 $-24.00(18)$ C1 C2 C3 C4 $0.5(4)$

Ru1 N1 C1 C2 $179.15(18)$ C9 C8 C7 C6 $\quad-56.6(3)$

Ru1 N1 C5 C4 178.81(19) C9 C10C11P1 154.78(17)

Ru1 N1 C5 C6 2.1(3) C2 C3 C4 C5 -1.0(4)

$\begin{array}{lllll}\text { Ru1 N2 C10C9 } 149.81(16) & \text { C3 C4 C5 N1 } & 0.9(4)\end{array}$

Ru1 N2 C10C11 $-28.5(3)$ C3 C4 C5 C6 $177.4(2)$

Ru1 N2 C6 C5 17.2(2) C4 C5 C6 N2 $171.6(2)$

Ru1 N2 C6 C7 145.10(16) C4 C5 C6 C7 $44.8(3)$

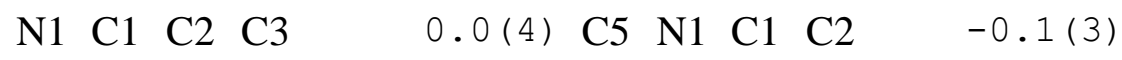

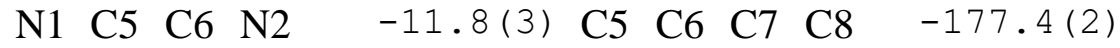

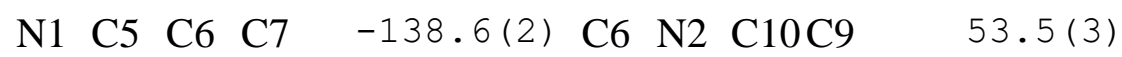

N2 $\mathrm{C} 10 \mathrm{C} 9 \mathrm{C} 8 \quad-53.6(3)$ C6 $\mathrm{N} 2 \mathrm{C} 10 \mathrm{C} 11 \quad 174.78(18)$

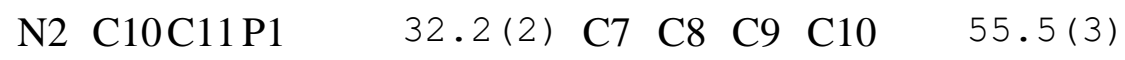

N2 $\quad \begin{array}{lllllll} & \mathrm{C} 7 & \mathrm{C} 8 & 57.8(3) & \mathrm{C} 11 \mathrm{P} 1 & \mathrm{C} 16 \mathrm{C} 18 & 164.52(16)\end{array}$

C16P1 C12C14 -43.3(2) C11P1 C16C17 $78.48(18)$

C16P1 C12C13 $162.50(16)$ C11P1 C16C19 $-40.2(2)$

C16P1 C12C15 81.40(18) C11P1 C12C14 66.81(19)

C16P1 C11C10 $146.06(17)^{-} \mathrm{C} 11 \mathrm{P} 1 \quad \mathrm{C} 12 \mathrm{C} 13-52.40(18)$

C12P1 C16C18 -52.84(18) C11P1 C12C15 $168.50(15)^{-}$

C12P1 C16C17 169.84(16) ${ }^{-} \mathrm{C} 11 \mathrm{C} 10 \mathrm{C} 9 \mathrm{C} 8 \quad-174.2(2)$ 
Table 7 Hydrogen Atom Coordinates $\left(\AA \times 10^{4}\right)$ and Isotropic Displacement Parameters $\left(\AA^{2} \times 10^{3}\right)$

Atom

H1

H14A

H14B

$\mathrm{H} 14 \mathrm{C}$

$\mathrm{H} 8 \mathrm{~A}$

H8B

$\mathrm{H} 10$

$\mathrm{H} 18 \mathrm{~A}$

H18B

H18C

H1A

H9A

H9B

$\mathrm{H} 2$

H3

H13A

H13B

H13C

$\mathrm{H} 4$

H15A

H15B

H15C

H17A

H17B

H17C

H6

H19A

H19B

H19C

H7A

H7B

H11A

H11B

\section{$x$}

7600 (16)

9934.59

10507.75

9950.33

6614.37

7216.17

8132.68

8059.94

8178.95

8950.38

5602.55

7854.64

7036.34

4515.48

4306.89

8910.41

9901.16

9361.5

5211.52

9214.99

9814.55

8822.93

7007.25

7099.95

6835.05

6930.22

9207.43

8532.1

8430.93

5841.49

5780.06

7881.09

8748.54

\section{$y$}

$9100(30)$

5459.3

7034.23

6890.34

1582.79

2895.64

4751.27

8794.36

7740.47

8011.95

10306.32

2001.24

2452

10579.23

8533.96

7773.37

7705.54

6064.06

6302.92

9533.49

9775.46

9869.28

4954.95

5744.51

6861.87

5830.22

5038.52

4748.3

3849.25

3934.5

3693.78

3836.98

4179.09 $z$

$3733(17)$

3605.71

4007.41

2927.05

4530.21

5237.14

4656.2

1474.53

687.76

1655.28

2846.77

4265.5

3371.11

3316.09

4232.29

4776.64

5090.84

4799.91

4671.63

2883.1

3945.6

3619.05

1374.26

498.85

1163.11

4930.62

2007.65

980.4

1823.91

4623.23

3595.46

2833.11

3686.88
U(eq)

47 ( 8 )

49

49

49

43

43

31

47

47

47

34

42

42

43

54

52

52

52

49

49

49

49

50

50

50

33

52

52

52

41

41

32

32 
Western Univeristy Sample Code b20088

X-ray Structure Report for complex 6 crystallized from hexane, CCDC \#2008083

\author{
Prepared by \\ Louise N. Dawe, PhD \\ Department of Chemistry and Biochemistry \\ Wilfrid Laurier University \\ Science Building \\ 75 University Ave. W. \\ Waterloo, ON, ON \\ ldawe@wlu.ca
}

February 25, 2020

Introduction

Dr. Paul D. Boyle, Department of Chemistry X-Ray Facility, Western University, is acknowledged for assistance with X-ray data collection.

$\mathrm{H}$-atoms, except $\mathrm{H} 1, \mathrm{H} 2 \mathrm{H} 3$, and $\mathrm{H} 3 \mathrm{~A}$, were introduced in calculated positions and refined on a riding model. All other atoms were introduced in difference map positions and refined isotropically $(\mathrm{H} 1, \mathrm{H} 2 \mathrm{H} 3$, and $\mathrm{H} 3 \mathrm{~A})$ or anisotropically (all non-hydrogen atoms.)

$\mathrm{H} 1$ and $\mathrm{H} 2$ are singly-bonded Ru-bound hydrides. Distance restraints with default standard uncertainty (SHELX DFIX), and a target distance based on the average singly bonded $\mathrm{Ru}-\mathrm{H}$ bond from a survey of the Cambridge Structural Database were employed for Ru1-H1 and Ru2-H2 bond lengths. A distance restraint was also used for the N3-H3A bond (where H3A was introduced from its difference map position and was allowed to refine positionally and isotropically).

Disordered lattice solvent hexane was present and crossed a symmetry element. A model that made physical sense could not be determined, despite multiple attempts. PLATON's SQUEEZE [1] procedure was employed to address this electron contribution via a solvent mask, resulting in excellent refinement statistics. This electron contribution was equivalent to 91 electrons per formula unit (less than two full molecules of hexane; occupancy for the disordered solvent could not be readily determined prior to the application of the solvent mask). 


\section{Experimental}

A single crystal of $\mathrm{C}_{40} \mathrm{H}_{66} \mathrm{~N}_{4} \mathrm{O}_{2} \mathrm{P}_{2} \mathrm{Ru}_{2}$ was selected and collected on a Bruker APEX-II CCD diffractometer. The crystal was kept at 110(2) K during data collection. Using Olex2 [1], the structure was solved with the ShelXT [2] structure solution program using Direct Methods and refined with the ShelXL [3] refinement package using Least Squares minimisation.

\section{Crystal structure determination}

Crystal Data for $\mathrm{C}_{40} \mathrm{H}_{66} \mathrm{~N}_{4} \mathrm{O}_{2} \mathrm{P}_{2} \mathrm{Ru}_{2}(M=899.04 \mathrm{~g} / \mathrm{mol})$ : orthorhombic, space group Pbca (no. 61), $a=$ 15.494(2) $\AA, b=20.027(3) \AA, c=30.174(5) \AA, V=9363(2) \AA^{3}, Z=8, T=110(2) \mathrm{K}, \mu(\mathrm{MoK \alpha})=0.747 \mathrm{~mm}^{-}$ ${ }^{1}$, Dcalc $=1.276 \mathrm{~g} / \mathrm{cm}^{3}, 197606$ reflections measured $\left(4.882^{\circ} \leq 2 \theta \leq 51.364^{\circ}\right), 8868$ unique (8290 with $\mathrm{I}>$ $\left.2 \sigma(\mathrm{I}) ; R_{\text {int }}=0.0305, \mathrm{R}_{\text {sigma }}=0.0104\right)$ which were used in all calculations. The final $R_{1}$ was $0.0259(\mathrm{I}>2 \sigma(\mathrm{I}))$ and $w R_{2}$ was 0.0753 (all data). 
Table 1 Crystal data and structure refinement

Identification code

Empirical formula

Formula weight

Temperature/K

Crystal system

Space group

$\mathrm{a} / \AA$

$\mathrm{b} / \AA$

$\mathrm{c} / \AA$

$\alpha /^{\circ}$

$\beta /{ }^{\circ}$

$\gamma /{ }^{\circ}$

Volume $/ \AA^{3}$

Z

$\rho_{\text {calc } g / \mathrm{cm}^{3}}$

$\mu / \mathrm{mm}^{-1}$

$\mathrm{F}(000)$

Crystal size $/ \mathrm{mm}^{3}$

Radiation b20088 (Western Xray Code)

$\mathrm{C}_{40} \mathrm{H}_{66} \mathrm{~N}_{4} \mathrm{O}_{2} \mathrm{P}_{2} \mathrm{Ru}_{2}$

899.04

110(2)

orthorhombic

Pbca

$15.494(2)$

20.027(3)

$30.174(5)$

90

90

90

9363(2)

8

1.276

0.747

3744.0

$0.429 \times 0.273 \times 0.248$

$\operatorname{MoK} \alpha(\lambda=0.71073)$

$2 \Theta$ range for data collection $/{ }^{\circ} 4.882$ to 51.364

Index ranges

Reflections collected

Independent reflections

Data/restraints/parameters

Goodness-of-fit on $\mathrm{F}^{2}$

Final $R$ indexes $[\mathrm{I}>=2 \sigma(\mathrm{I})] \quad \mathrm{R}_{1}=0.0259, \mathrm{wR}_{2}=0.0731$

Final $\mathrm{R}$ indexes [all data] $\quad \mathrm{R}_{1}=0.0284, \mathrm{wR}_{2}=0.0753$

Largest diff. peak/hole / e $\AA^{-3} 0.84 /-0.65$
$-18 \leq \mathrm{h} \leq 18,-24 \leq \mathrm{k} \leq 23,-36 \leq 1 \leq 36$

197606

8868 [8290 with I $>2 \sigma(I) ; R_{\text {int }}=0.0305, R_{\text {sigma }}=0.0104$ ]

$8868 / 3 / 479$

1.126

$\mathrm{R}_{1}=0.0259, \mathrm{wR}_{2}=0.0731$
$\mathrm{R}_{1}=0.0284, \mathrm{wR}_{2}=0.0753$ 
Table 2 Fractional Atomic Coordinates $\left(\times 10^{4}\right)$ and Equivalent Isotropic Displacement Parameters $\left(\AA^{2} \times 10^{3}\right)$. Ueq is defined as $1 / 3$ of of the trace of the orthogonalised UIJ tensor.

\begin{tabular}{|c|c|c|c|c|}
\hline Atom & $x$ & $y$ & $z$ & $\mathbf{U}(\mathbf{e q})$ \\
\hline Ru1 & $5632.7(2)$ & $5959.8(2)$ & $6406.6(2)$ & $14.42(6)$ \\
\hline $\mathrm{Ru} 2$ & $6806.1(2)$ & $6911.5(2)$ & $5671.1(2)$ & $16.49(6)$ \\
\hline $\mathrm{P} 1$ & $5613.2(4)$ & $5166.8(3)$ & $6957.7(2)$ & $18.67(13)$ \\
\hline $\mathrm{P} 2$ & $6024.6(4)$ & $6849.3(3)$ & $5036.0(2)$ & $18.07(13)$ \\
\hline $\mathrm{O} 1$ & $4525.9(14)$ & $5157.0(10)$ & $5792.4(7)$ & $34.6(5)$ \\
\hline $\mathrm{O} 2$ & $6409.4(17)$ & $8352.3(10)$ & $5852.3(7)$ & $46.2(6)$ \\
\hline N1 & $4715.9(13)$ & $6735.5(10)$ & $6599.6(6)$ & $18.0(4)$ \\
\hline $\mathrm{N} 2$ & $6293.9(13)$ & $6519.8(9)$ & $6903.9(6)$ & $17.5(4)$ \\
\hline N3 & $7709.0(13)$ & $6641.7(10)$ & $6191.7(7)$ & $20.7(4)$ \\
\hline N4 & $7144.8(13)$ & $5933.9(10)$ & $5492.8(6)$ & $19.0(4)$ \\
\hline $\mathrm{C} 1$ & $3872.4(16)$ & $6757.2(12)$ & $6492.2(8)$ & $21.0(5)$ \\
\hline $\mathrm{C} 2$ & $3338.6(17)$ & $7284.3(14)$ & $6597.5(8)$ & $27.6(6)$ \\
\hline C3 & $3685(2)$ & $7819.4(16)$ & $6820.3(11)$ & $41.6(7)$ \\
\hline $\mathrm{C} 4$ & $4558(2)$ & $7811.3(15)$ & $6924.2(10)$ & $36.4(7)$ \\
\hline $\mathrm{C} 5$ & $5063.1(16)$ & $7265.1(12)$ & $6812.2(8)$ & $20.9(5)$ \\
\hline C6 & $6041.2(16)$ & $7227.0(11)$ & $6873.9(8)$ & $20.0(5)$ \\
\hline $\mathrm{C} 7$ & $6400.4(17)$ & $7667.2(13)$ & $7244.9(8)$ & $25.5(5)$ \\
\hline $\mathrm{C} 8$ & $6255.6(18)$ & $7353.5(13)$ & $7699.2(8)$ & $27.3(6)$ \\
\hline C9 & $6686.7(18)$ & $6670.2(13)$ & $7700.6(8)$ & $27.7(6)$ \\
\hline $\mathrm{C} 10$ & $6353.7(16)$ & $6243.2(12)$ & $7319.3(8)$ & $23.0(5)$ \\
\hline C11 & $6154.9(18)$ & $5592.9(12)$ & $7394.3(8)$ & $24.6(5)$ \\
\hline $\mathrm{C} 12$ & $4550.2(17)$ & $4894.4(13)$ & $7219.3(9)$ & $26.9(6)$ \\
\hline $\mathrm{C} 13$ & $3976.5(19)$ & $5517.1(14)$ & $7221.7(10)$ & $33.8(6)$ \\
\hline $\mathrm{C} 14$ & $4642(2)$ & $4665.4(14)$ & $7704.2(9)$ & $35.7(7)$ \\
\hline $\mathrm{C} 15$ & $4091(2)$ & $4359.9(15)$ & $6942.2(11)$ & $39.4(7)$ \\
\hline $\mathrm{C} 16$ & $6276.7(16)$ & $4386.1(12)$ & $6840.4(8)$ & $22.5(5)$ \\
\hline $\mathrm{C} 17$ & $7225.1(17)$ & $4602.0(14)$ & $6809.6(9)$ & $29.3(6)$ \\
\hline $\mathrm{C} 18$ & $6021.4(19)$ & $4071.7(13)$ & $6396.3(9)$ & $27.5(6)$ \\
\hline C19 & $6231(2)$ & $3859.5(13)$ & $7209.3(9)$ & $33.8(6)$ \\
\hline $\mathrm{C} 20$ & $4979.1(16)$ & $5473.6(12)$ & $6024.6(8)$ & $21.4(5)$ \\
\hline $\mathrm{C} 21$ & $8208.5(17)$ & $7184.9(13)$ & $6403.5(9)$ & $25.9(5)$ \\
\hline $\mathrm{C} 22$ & $8805.0(19)$ & $6923.1(14)$ & $6764.8(9)$ & $32.6(6)$ \\
\hline $\mathrm{C} 23$ & $9413.7(17)$ & $6392.6(15)$ & $6580.6(10)$ & $31.8(6)$ \\
\hline $\mathrm{C} 24$ & $8903.7(17)$ & $5841.3(14)$ & $6345.0(9)$ & $28.0(6)$ \\
\hline $\mathrm{C} 25$ & $8310.8(16)$ & $6139.4(13)$ & $5996.3(9)$ & $24.1(5)$ \\
\hline $\mathrm{C} 26$ & $7809.7(16)$ & $5651.2(13)$ & $5713.7(8)$ & $22.2(5)$ \\
\hline $\mathrm{C} 27$ & $8008.9(17)$ & $4983.0(13)$ & $5654.4(8)$ & $25.9(5)$ \\
\hline $\mathrm{C} 28$ & $7527.3(18)$ & $4611.2(13)$ & $5352.4(9)$ & $28.2(5)$ \\
\hline $\mathrm{C} 29$ & $6864.8(17)$ & $4911.3(13)$ & $5119.3(8)$ & $25.3(5)$ \\
\hline $\mathrm{C} 30$ & $6673.3(15)$ & $5578.5(12)$ & $5201.2(8)$ & $20.2(5)$ \\
\hline C31 & $5921.5(16)$ & $5933.0(12)$ & $4991.0(8)$ & $20.8(5)$ \\
\hline $\mathrm{C} 32$ & $6615.2(17)$ & $7096.7(14)$ & $4511.5(8)$ & $26.4(5)$ \\
\hline
\end{tabular}




$\begin{array}{lllll}\text { C33 } 3094.1(19) & 7002.8(17) & 4080.9(9) & 35.0(7) \\ \text { C34 } & 7416.4(17) & 6642.5(15) & 4477.5(9) & 31.1(6) \\ \text { C35 } & 6914.4(18) & 7820.0(15) & 4547.4(9) & 31.8(6) \\ \text { C36 } & 4873.4(15) & 7148.8(13) & 5003.1(8) & 22.1(5) \\ \text { C37 } & 4318.9(16) & 6765.5(14) & 4664.8(9) & 28.3(6) \\ \text { C38 } & 4469.0(16) & 7051.3(14) & 5462.2(9) & 26.4(5) \\ \text { C39 } & 4849.8(17) & 7898.3(13) & 4901.4(9) & 28.1(6) \\ \text { C40 } & 6557.1(18) & 7788.8(13) & 5790.7(8) & 27.3(6)\end{array}$


Table 3 Anisotropic Displacement Parameters $\left(\AA^{2} \times 10^{3}\right)$. The Anisotropic displacement factor exponent takes the form: $-2 \pi^{2}\left[h^{2} a^{* 2} U_{11}+2 h k a * b^{*} U_{12}+\ldots\right]$.

\begin{tabular}{|c|c|c|c|c|c|c|}
\hline Atom & $\mathbf{U}_{11}$ & $\mathbf{U}_{22}$ & $\mathbf{U}_{33}$ & $\mathbf{U}_{23}$ & $\mathbf{U}_{13}$ & $\mathbf{U}_{12}$ \\
\hline Ru1 & $16.71(10)$ & $12.27(10)$ & $14.28(10)$ & $-1.72(6)$ & $-0.02(7)$ & $-1.87(6)$ \\
\hline $\mathrm{Ru} 2$ & $15.82(10)$ & $17.99(10)$ & $15.66(10)$ & $-0.49(7)$ & $-0.11(7)$ & $-4.24(7)$ \\
\hline $\mathrm{P} 1$ & $24.5(3)$ & $13.8(3)$ & $17.7(3)$ & $0.5(2)$ & $1.7(2)$ & $-1.0(2)$ \\
\hline $\mathrm{P} 2$ & $16.0(3)$ & $22.4(3)$ & $15.8(3)$ & $1.1(2)$ & $0.1(2)$ & $-2.9(2)$ \\
\hline $\mathrm{O} 1$ & $44.7(12)$ & $29.1(10)$ & $30.0(10)$ & $-6.7(8)$ & $-12.0(9)$ & $-9.9(9)$ \\
\hline $\mathrm{O} 2$ & $78.2(17)$ & $18.5(10)$ & $41.9(12)$ & $0.2(9)$ & $-3.6(12)$ & $-4.9(10)$ \\
\hline N1 & $20.5(10)$ & $17.9(9)$ & $15.5(9)$ & $1.0(7)$ & $2.5(8)$ & $-1.2(8)$ \\
\hline $\mathrm{N} 2$ & $20.8(10)$ & $15.4(9)$ & $16.3(9)$ & $-1.1(7)$ & $-1.0(8)$ & $-2.1(8)$ \\
\hline N3 & $17.1(10)$ & $23.7(10)$ & $21.3(10)$ & $-1.0(8)$ & $-0.8(8)$ & $-4.4(8)$ \\
\hline N4 & $14.8(9)$ & $24.7(10)$ & $17.4(10)$ & $-1 \cdot 3(8)$ & $2.1(8)$ & $-1.1(8)$ \\
\hline $\mathrm{C} 1$ & $22.6(12)$ & $24.0(12)$ & $16.4(11)$ & $3.0(9)$ & $2.0(9)$ & $-1.6(10)$ \\
\hline $\mathrm{C} 2$ & $23.9(13)$ & $37.5(15)$ & $21.3(12)$ & $0.0(11)$ & $3.3(10)$ & $7.4(11)$ \\
\hline $\mathrm{C} 3$ & $37.7(16)$ & $40.3(17)$ & $47.0(18)$ & $-17.8(14)$ & $-3.2(14)$ & $18.3(14)$ \\
\hline $\mathrm{C} 4$ & $37.3(16)$ & $30.3(15)$ & $41.6(16)$ & $-18.7(13)$ & $-6.3(13)$ & $6.6(12)$ \\
\hline $\mathrm{C} 5$ & $27.0(13)$ & $19.5(11)$ & $16.1(11)$ & $-1.9(9)$ & $2.1(9)$ & $0.1(10)$ \\
\hline C6 & $25.0(12)$ & $15.8(11)$ & $19.3(11)$ & $-2.1(9)$ & $0.5(9)$ & $-3.2(9)$ \\
\hline $\mathrm{C} 7$ & $29.0(13)$ & $21.2(12)$ & $26.3(13)$ & $-5.3(10)$ & $-3.1(11)$ & $-4.8(10)$ \\
\hline $\mathrm{C} 8$ & $32.2(14)$ & $28.4(14)$ & $21.2(12)$ & $-9.9(10)$ & $-2.2(11)$ & $-4.5(11)$ \\
\hline C9 & $36.0(15)$ & $28.2(13)$ & $18.7(12)$ & $-3.6(10)$ & $-7.2(11)$ & $-2.9(11)$ \\
\hline $\mathrm{C} 10$ & $26.4(13)$ & $23.6(12)$ & $18.9(11)$ & $-3.4(10)$ & $-2.4(10)$ & $0.3(10)$ \\
\hline C11 & $37.8(15)$ & $21.9(12)$ & $14.3(11)$ & $2.3(9)$ & $-3.7(10)$ & $2.3(11)$ \\
\hline $\mathrm{C} 12$ & $29.3(13)$ & $20.3(12)$ & $31.2(14)$ & $4.7(10)$ & $9.9(11)$ & $-1.4(10)$ \\
\hline C13 & $32.6(15)$ & $30.1(14)$ & $38.6(16)$ & $10.4(12)$ & $15.3(12)$ & $4.7(12)$ \\
\hline C14 & $48.1(17)$ & $25.3(14)$ & $33.7(15)$ & $9.3(11)$ & $18.4(13)$ & $4.5(12)$ \\
\hline $\mathrm{C} 15$ & $36.5(16)$ & $28.9(15)$ & $52.8(19)$ & $0.9(13)$ & $10.5(14)$ & $-12 \cdot 3(12)$ \\
\hline C16 & $26.9(13)$ & $16.2(11)$ & $24.3(12)$ & $0.8(9)$ & $0.1(10)$ & $2.1(9)$ \\
\hline $\mathrm{C} 17$ & $25.6(13)$ & $28.2(14)$ & $34.2(14)$ & $1.5(11)$ & $-3.8(11)$ & $4.0(11)$ \\
\hline C18 & $33.4(15)$ & $20.4(13)$ & $28.8(14)$ & $-6.0(10)$ & $0.7(11)$ & $2.0(11)$ \\
\hline C19 & $47.2(17)$ & $19.9(13)$ & $34.2(15)$ & $5.3(11)$ & $5.3(13)$ & $6.9(12)$ \\
\hline $\mathrm{C} 20$ & $27.1(13)$ & $17.3(11)$ & $19.8(11)$ & $0.8(9)$ & $2.0(10)$ & $-1.1(10)$ \\
\hline $\mathrm{C} 21$ & $23.4(13)$ & $26.9(13)$ & $27.5(13)$ & $-4.2(10)$ & $-4.2(10)$ & $-4.4(10)$ \\
\hline $\mathrm{C} 22$ & $30.8(15)$ & $36.7(15)$ & $30.3(14)$ & $-1.5(12)$ & $-10.1(12)$ & $-5.3(12)$ \\
\hline $\mathrm{C} 23$ & $23.6(13)$ & $36.5(15)$ & $35.3(15)$ & $4.8(12)$ & $-9.4(11)$ & $-4.5(11)$ \\
\hline $\mathrm{C} 24$ & $22.3(13)$ & $29.9(14)$ & $31.9(14)$ & $4.6(11)$ & $-5.7(11)$ & $-1.8(11)$ \\
\hline $\mathrm{C} 25$ & $18.7(12)$ & $27.8(13)$ & $25.7(13)$ & $-0.1(10)$ & $-1.1(10)$ & $-0.3(10)$ \\
\hline $\mathrm{C} 26$ & $18.0(12)$ & $29.9(13)$ & $18.5(11)$ & $-1.0(10)$ & $3.1(9)$ & $-0.1(10)$ \\
\hline $\mathrm{C} 27$ & $22.4(12)$ & $29.7(14)$ & $25.4(13)$ & $1.2(10)$ & $0.6(10)$ & $5.6(10)$ \\
\hline C28 & $30.5(14)$ & $21.9(12)$ & $32.3(14)$ & $-3.3(11)$ & $3.4(11)$ & $1.5(11)$ \\
\hline $\mathrm{C} 29$ & $27.4(13)$ & $24.6(13)$ & $23.8(13)$ & $-4 \cdot 3(10)$ & $-0.6(10)$ & $-2.6(10)$ \\
\hline C30 & $18.8(11)$ & $24.7(12)$ & $17.0(11)$ & $-2.1(9)$ & $1.9(9)$ & $-3.9(9)$ \\
\hline C31 & $20.5(12)$ & $23.3(12)$ & $18.6(11)$ & $-3.1(9)$ & $-1.5(9)$ & $-2.3(9)$ \\
\hline C32 & $22.7(12)$ & $40.3(15)$ & $16.3(12)$ & $2.7(11)$ & $2.6(10)$ & $-5.8(11)$ \\
\hline
\end{tabular}




$\begin{array}{lrrrrrr}\text { C33 } & 30.6(15) & 57.2(19) & 17.2(12) & 4.1(12) & 0.6(11) & -6.2(13) \\ \text { C34 } & 23.6(13) & 47.4(17) & 22.4(12) & -0.2(12) & 4.7(11) & -2.7(12) \\ \text { C35 } & 24.9(13) & 43.1(16) & 27.5(14) & 10.0(12) & 3.5(11) & -8.8(12) \\ \text { C36 } & 17.5(11) & 27.9(13) & 20.8(12) & 3.8(10) & 0.0(9) & -0.3(10) \\ \text { C37 } & 20.8(13) & 36.1(15) & 27.9(13) & 3.0(11) & -4.4(10) & -1.9(11) \\ \text { C38 } & 18.9(12) & 33.7(14) & 26.6(13) & 5.0(11) & 3.9(10) & 3.5(10) \\ \text { C39 } & 25.2(13) & 28.4(13) & 30.9(14) & 6.6(11) & 0.0(11) & 1.4(11) \\ \text { C40 } & 35.4(14) & 25.0(14) & 21.6(12) & 2.7(10) & -0.8(11) & -8.3(11)\end{array}$




\section{Table 4 Bond Lengths}

Atom Atom Length/Å Atom Atom Length/Å

\begin{tabular}{|c|c|c|c|c|c|}
\hline $\mathrm{Ru} 1$ & $\mathrm{P} 1$ & $2.2996(7)$ & $\mathrm{C} 5$ & C6 & $1.529(3)$ \\
\hline $\mathrm{Ru} 1$ & N1 & $2.184(2)$ & $\mathrm{C} 6$ & $\mathrm{C} 7$ & $1.530(3)$ \\
\hline Ru1 & $\mathrm{N} 2$ & $2.1352(19)$ & $\mathrm{C} 7$ & $\mathrm{C} 8$ & $1.524(4)$ \\
\hline Ru1 & $\mathrm{C} 20$ & $1.817(2)$ & $\mathrm{C} 8$ & C9 & $1.523(4)$ \\
\hline $\mathrm{Ru} 2$ & $\mathrm{P} 2$ & $2.2704(7)$ & C9 & $\mathrm{C} 10$ & $1.524(3)$ \\
\hline $\mathrm{Ru} 2$ & N3 & $2.172(2)$ & $\mathrm{C} 10$ & $\mathrm{C} 11$ & $1.357(4)$ \\
\hline $\mathrm{Ru} 2$ & N4 & $2.097(2)$ & $\mathrm{C} 12$ & $\mathrm{C} 13$ & $1.532(4)$ \\
\hline $\mathrm{Ru} 2$ & $\mathrm{C} 40$ & $1.835(3)$ & $\mathrm{C} 12$ & $\mathrm{C} 14$ & $1.540(4)$ \\
\hline $\mathrm{P} 1$ & $\mathrm{C} 11$ & $1.780(3)$ & $\mathrm{C} 12$ & $\mathrm{C} 15$ & $1.534(4)$ \\
\hline $\mathrm{P} 1$ & $\mathrm{C} 12$ & $1.906(3)$ & $\mathrm{C} 16$ & $\mathrm{C} 17$ & $1.535(4)$ \\
\hline $\mathrm{P} 1$ & $\mathrm{C} 16$ & $1.904(2)$ & $\mathrm{C} 16$ & $\mathrm{C} 18$ & $1.533(3)$ \\
\hline $\mathrm{P} 2$ & C31 & $1.847(2)$ & $\mathrm{C} 16$ & $\mathrm{C} 19$ & $1.535(3)$ \\
\hline $\mathrm{P} 2$ & C32 & $1.894(3)$ & $\mathrm{C} 21$ & $\mathrm{C} 22$ & $1.522(4)$ \\
\hline $\mathrm{P} 2$ & C36 & $1.885(3)$ & $\mathrm{C} 22$ & $\mathrm{C} 23$ & $1.525(4)$ \\
\hline $\mathrm{O} 1$ & $\mathrm{C} 20$ & $1.177(3)$ & $\mathrm{C} 23$ & $\mathrm{C} 24$ & $1.533(4)$ \\
\hline $\mathrm{O} 2$ & $\mathrm{C} 40$ & $1.166(3)$ & $\mathrm{C} 24$ & $\mathrm{C} 25$ & $1.519(3)$ \\
\hline N1 & $\mathrm{C} 1$ & $1.347(3)$ & $\mathrm{C} 25$ & $\mathrm{C} 26$ & $1.512(3)$ \\
\hline N1 & $\mathrm{C} 5$ & $1.351(3)$ & $\mathrm{C} 26$ & $\mathrm{C} 27$ & $1.385(4)$ \\
\hline $\mathrm{N} 2$ & C6 & $1.472(3)$ & $\mathrm{C} 27$ & $\mathrm{C} 28$ & $1.393(4)$ \\
\hline $\mathrm{N} 2$ & $\mathrm{C} 10$ & $1.374(3)$ & $\mathrm{C} 28$ & $\mathrm{C} 29$ & $1.382(4)$ \\
\hline N3 & $\mathrm{C} 21$ & $1.480(3)$ & C29 & C30 & $1.391(4)$ \\
\hline N3 & $\mathrm{C} 25$ & $1.493(3)$ & C30 & C31 & $1.504(3)$ \\
\hline $\mathrm{N} 4$ & $\mathrm{C} 26$ & $1.351(3)$ & C32 & C33 & $1.541(4)$ \\
\hline $\mathrm{N} 4$ & C30 & $1.347(3)$ & C32 & C34 & $1.543(4)$ \\
\hline $\mathrm{C} 1$ & $\mathrm{C} 2$ & $1.378(4)$ & C32 & C35 & $1.525(4)$ \\
\hline $\mathrm{C} 2$ & $\mathrm{C} 3$ & $1.374(4)$ & C36 & C37 & $1.539(4)$ \\
\hline $\mathrm{C} 3$ & $\mathrm{C} 4$ & $1.389(4)$ & C36 & C38 & $1.533(3)$ \\
\hline $\mathrm{C} 4$ & $\mathrm{C} 5$ & $1.387(4)$ & C36 & C39 & $1.533(3)$ \\
\hline
\end{tabular}


Table 5 Bond Angles

\section{Atom Atom Atom}

N1 Ru1 P1

N2 Ru1 P1

N2 Ru1 N1

C20 Ru1 P1

C20 Ru1 N1

C20 Ru1 N2

N3 Ru2 P2

N4 Ru2 P2

N4 Ru2 N3

C40 Ru2 P2

C40 Ru2 N3

C40 Ru2 N4

C11 P1 Ru1

C11 P1 C12

C11 P1 C16

C12 P1 Ru1

C16 P1 Ru1

C16 P1 C12

C31 P2 Ru2

C31 P2 C32

C31 P2 C36

C32 P2 Ru2

C36 P2 Ru2

C36 P2 C32

C1 N1 Ru1

C1 N1 C5

C5 N1 Ru1

C6 N2 Ru1

C10 N2 Ru1

C10 N2 C6

C21 N3 Ru2

C21 N3 C25

C25 N3 Ru2

C26 N4 Ru2

C30 N4 Ru2

C30 N4 C26

$\mathrm{N} 1 \mathrm{C} 1 \mathrm{C} 2$

$\mathrm{C} 3 \quad \mathrm{C} 2 \mathrm{C} 1$

$\mathrm{C} 2 \quad \mathrm{C} 3 \quad \mathrm{C} 4$

$\mathrm{C} 5 \mathrm{C} 4 \mathrm{C} 3$

$\mathrm{N} 1 \quad \mathrm{C} 5 \quad \mathrm{C} 4$

N1 C5 C6

$\mathrm{C} 4 \mathrm{C} 5 \mathrm{C} 6$

\section{Angle $/^{\circ}$}

$106.85(5)$

$82.00(5) \mathrm{N} 2$

$75.59(7)$

$94.66(8)$

$100.82(9)$

$174.04(9)$

$160.13(6)$

$82.28(6)$

$78.00(8)$

$96.11(8)$

$103.38(10)$

$\mathrm{C} 15$

$\mathrm{C} 15$

$175.64(10)$

$\mathrm{C} 17$

$101.42(8)$

$\mathrm{C} 17$

$103.77(12)$

C18

$106.05(12)$

$\mathrm{C} 18$

$120.57(9)$

$\mathrm{C} 18$

$115.19(8)$

C19

$107.96(11)$

$\mathrm{O} 1$

$99.36(8) \quad \mathrm{N} 3$

$103.91(12)$

$\mathrm{C} 21$

$103.32(11)$

$\mathrm{C} 22$

$115.68(8)$

$\mathrm{C} 25$

$\mathrm{C} 23 \mathrm{C} 24$

$122.12(8) \quad \mathrm{N} 3 \quad \mathrm{C} 25 \quad \mathrm{C} 24$

$109.27(11) \quad \mathrm{N} 3 \quad \mathrm{C} 25 \quad \mathrm{C} 26$

126.14(16) C26 C25 C24

118.4(2) N4 C26 C25

$115.21(16) \quad \mathrm{N} 4 \quad \mathrm{C} 26 \quad \mathrm{C} 27$

109.55(14) C27 C26 C25

117.51(15) C26 C27 C28

117.51(19) $\mathrm{C} 29 \quad \mathrm{C} 28 \quad \mathrm{C} 27$

117.79(16) C28 C29 C30

$109.82(19) \quad \mathrm{N} 4 \quad \mathrm{C} 30 \quad \mathrm{C} 29$

$106.52(15) \quad \mathrm{N} 4 \quad \mathrm{C} 30 \quad \mathrm{C} 31$

117.09(16) C29 C30 $\quad$ C31

121.68(16) C30 C31 P2

120.9(2) C33 C32 P2

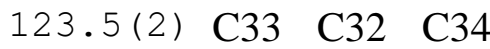

118.4(3) $\mathrm{C} 34 \quad \mathrm{C} 32 \quad \mathrm{P} 2$

118.8(3) C35 C32 P2

$120.3(3) \quad$ C35 $\quad$ C32 $\quad$ C33

120.7(2) $\mathrm{C} 35 \quad \mathrm{C} 32 \quad \mathrm{C} 34$

$114.4(2) \quad \mathrm{C} 37 \quad \mathrm{C} 36 \quad \mathrm{P} 2$

124.7(2) C38 C36 P2
Angle ${ }^{\circ}$

110.7 (2)

$119.1(2)$

$121.6(2)$

$119.3(2)$

$116.32(18)$

$105.68(17)$

$107.0(2)$

$107.5(2)$

$113.5(2)$

$112.02(19)$

$110.7(2)$

$107.27(17)$

$106.4(2)$

$111.13(17)$

$108.1(2)$

$109.9(2)$

$113.87(18)$

$176.8(2)$

$111.9(2)$

$110.8(2)$

$110.6(2)$

$110.5(2)$

$111.7(2)$

$109.74(19)$

$116.5(2)$

$113.5(2)$

$120.7(2)$

125.7 (2)

$118.8(2)$

$119.9(2)$

$119.1(2)$

$120.5(2)$

116.5 (2)

$122.9(2)$

111.77 (17)

$114.80(18)$

$107.1(2)$

$106.87(18)$

$109.64(18)$

$109.6(2)$

$108.7(2)$

$113.86(18)$

$107.39(16)$ 


$\begin{array}{lllrlllr}\text { N2 } & \text { C6 } & \text { C5 } & 108.61(19) & \text { C38 } & \text { C36 } & \text { C37 } & 107.9(2) \\ \text { N2 } & \text { C6 } & \text { C7 } & 114.4(2) & \text { C39 } & \text { C36 } & \text { P2 } & 110.17(17) \\ \text { C5 } & \text { C6 } & \text { C7 } & 114.9(2) & \text { C39 } & \text { C36 } & \text { C37 } & 110.0(2) \\ \text { C8 } & \text { C7 } & \text { C6 } & 111.5(2) & \text { C39 } & \text { C36 } & \text { C38 } & 107.2(2) \\ \text { C9 } & \text { C8 } & \text { C7 } & 108.0(2) & \text { O2 } & \text { C40 } & \text { Ru2 } & 177.6(2)\end{array}$


Table 6 Torsion Angles

\begin{tabular}{|c|c|c|c|c|}
\hline A $\mathbf{B}$ & C D & Angle $/^{\circ}$ & A B C & Angle $/^{\circ}$ \\
\hline Ru1 P1 & C11 C10 & $5.8(2)$ & C6 N2 C10C11 & $146.6(2)$ \\
\hline $11 \mathrm{~N}$ & $\mathrm{C} 1 \mathrm{C} 2$ & $175.23(18)$ & $\begin{array}{lllll}\text { C6 } & \text { C7 } & \text { C8 } & \text { C9 }\end{array}$ & $58.0(3)$ \\
\hline u1 N1 & $\mathrm{C} 5 \mathrm{C} 4$ & $175.5(2)$ & C7 C8 C9 C10 & $-55.3(3)$ \\
\hline u1 N1 & $\mathrm{C} 5 \mathrm{C} 6$ & $0.7(2)$ & C8 C9 C10N2 & $46.2(3)$ \\
\hline $\mathrm{u} 1 \mathrm{~N} 2$ & C6 C5 & $44.1(2)$ & C8 C9 C10C11 & $-136.3(3)$ \\
\hline $\mathrm{u} 1 \mathrm{~N} 2$ & C6 C7 & $173.88(16)$ & C9 C10C11P1 & $170.40(19)$ \\
\hline d1 N2 & C10C9 & $170.07(18)$ & $\mathrm{C} 10 \mathrm{~N} 2 \mathrm{C} 6 \mathrm{C} 5$ & $-93.5(2)$ \\
\hline $\mathrm{u} 1 \mathrm{~N} 2$ & C10C11 & $12.5(3)$ & C10N2 C6 C7 & $36.4(3)$ \\
\hline $\mathrm{u} 2 \mathrm{P} 2$ & C31 C30 & $-28.77(17)$ & C12P1 C11 C10 & $-119.9(2)$ \\
\hline $\mathrm{u} 2 \mathrm{P} 2$ & C32 C33 & $175.82(18)$ & C16P1 C11 C10 & $126.4(2)$ \\
\hline $\mathrm{u} 2 \mathrm{P} 2$ & C32 C34 & $57.3(2)$ & $\mathrm{C} 21 \mathrm{~N} 3 \mathrm{C} 25 \mathrm{C} 24$ & $58.9(3)$ \\
\hline $\mathrm{u} 2 \mathrm{P} 2$ & C32C35 & $-60.3(2)$ & $\mathrm{C} 21 \mathrm{~N} 3 \mathrm{C} 25 \mathrm{C} 26$ & -170 \\
\hline $2 \mathrm{P} 2$ & C36 C37 & $149.85(15)$ & $\mathrm{C} 21 \mathrm{C} 22 \mathrm{C} 23$ & $-53.4(3)$ \\
\hline $12 \mathrm{P} 2$ & C36 C38 & $-30.4(2)$ & $\mathrm{C} 24 \mathrm{C} 25$ & $53.7(3)$ \\
\hline $\mathrm{u} 2 \mathrm{P} 2$ & C36 C39 & $86.01(18)$ & $\mathrm{C} 23 \mathrm{C} 24 \mathrm{C} 25 \mathrm{~N} 3$ & $-56.9(3)$ \\
\hline $\mathrm{u} 2 \mathrm{~N} 3$ & $\mathrm{C} 21 \mathrm{C} 22$ & $179.31(17)$ & $\mathrm{C} 23 \mathrm{C} 24 \mathrm{C} 25 \mathrm{C} 26$ & 175 \\
\hline $2 \mathrm{~N} 3$ & $\mathrm{C} 25$ & $172.49(17)^{-}$ & $\mathrm{C} 2$ & $164.2(2)$ \\
\hline $2 \mathrm{~N} 3$ & $\mathrm{C} 25 \mathrm{C} 26$ & $-41.7(2)$ & $\mathrm{C} 24 \mathrm{C} 25 \mathrm{C} 26 \mathrm{C} 27$ & $-19.3(4)$ \\
\hline $2 \mathrm{~N} 4$ & $\mathrm{C} 26 \mathrm{C} 25$ & $-11.0(3)$ & $\mathrm{C} 25 \mathrm{~N} 3 \mathrm{C} 21 \mathrm{C} 22$ & $-58 \cdot 6(3)$ \\
\hline $2 \mathrm{~N} 4$ & $\mathrm{C} 26 \mathrm{C} 27$ & $172.25(18)$ & $\mathrm{C} 25 \mathrm{C} 26 \mathrm{C} 27 \mathrm{C} 28$ & $-174.4(2)$ \\
\hline $\mathrm{u} 2 \mathrm{~N} 4$ & $\mathrm{C} 30 \mathrm{C} 29$ & $174.03(18)^{-}$ & C26N4 C30C29 & $-0.4(3)$ \\
\hline 4218 & C30 C31 & $3.4(3)$ & C26N4 C30C31 & $177.1(2)$ \\
\hline $1 \mathrm{C} 1$ & C2 $\mathrm{C} 3$ & $0.6(4)$ & C26C27 C28 C29 & $0.0(4)$ \\
\hline $1 \mathrm{C} 5$ & C6 N2 & $-29.4(3)$ & C27 C28C29 C30 & $-2.0(4)$ \\
\hline N1 C5 & C6 C7 & $-158 \cdot 9(2)$ & C28C29C30 N4 & $2.2(4)$ \\
\hline N2 C6 & $\mathrm{C} 7 \mathrm{C} 8$ & $-48 \cdot 3(3)$ & C28C29C30C31 & $-175.1(2)$ \\
\hline $\mathrm{J} 2 \mathrm{C} 10$ & 0C11 P1 & $-12 \cdot 2(3)$ & C29C30C31 P2 & $-163.5(2)$ \\
\hline $\mathrm{N} 3 \mathrm{C}$ & $1 \mathrm{C} 22 \mathrm{C} 23$ & $56.5(3)$ & C30N4 C26C25 & $175.0(2)$ \\
\hline $3 \mathrm{C}^{\prime}$ & $5 \mathrm{C} 26 \mathrm{~N} 4$ & $36.0(3)$ & C30N4 C26C27 & (3) \\
\hline $\mathrm{J} 3 \mathrm{C} 25$ & $5 \mathrm{C} 26 \mathrm{C} 27$ & $-147.4(2)$ & C31 P2 C32 C33 & $68.1(2)$ \\
\hline V4 C26 & $6 \mathrm{C} 27 \mathrm{C}$ & $1.9(4)$ & C31 P2 C32 C34 & $-50.5(2)$ \\
\hline $4 \mathrm{C}$ & 0C31 P2 & $19.1(3)$ & C31 P2 C32 C35 & $168.11(18)$ \\
\hline $1 \mathrm{~N} 1$ & $\mathrm{C} 5 \mathrm{C} 4$ & $1.2(4)$ & C31P2 C36C37 & $-39.6(2)$ \\
\hline C1 N1 & C5 C6 & $-173.6(2)$ & C31 P2 C36 C38 & $79.80(19)$ \\
\hline $\mathrm{C} 2$ & $\mathrm{C} 3 \mathrm{C} 4$ & $0.9(5)$ & C31P2 C36C39 & $163.75(17)$ \\
\hline $\mathrm{C} 3$ & $\mathrm{C} 4 \mathrm{C} 5$ & $-1 \cdot 3(5)$ & C32P2 C31 C30 & $90.79(18)$ \\
\hline $3 \mathrm{C} 4$ & C5 N1 & $0.3(4)$ & C32P2 C36 C37 & $70.5(2)$ \\
\hline
\end{tabular}


C3 C4 C5 C6 $174.5(3)$ C32P2 C36C38 $170.05(18)$

$\begin{array}{lllllll}\text { C4 C5 C6 N2 } & 156.1(3) & \text { C32P2 C36C39 } & -53.6(2)\end{array}$

$\begin{array}{lllllllll}\mathrm{C} 4 & \mathrm{C} 5 & \mathrm{C} 6 & \mathrm{C} 7 & 26.6(4) & \mathrm{C} 36 \mathrm{P} 2 & \mathrm{C} 31 \mathrm{C} 30 & 155.13(17)\end{array}$



$\begin{array}{llllllll}\text { C5 } & \text { C6 } & \text { C7 } & \text { C8 } & 78.4(3) & \text { C36P2 } & \text { C32C34 } & 160.25(17)\end{array}$

$\begin{array}{llllll}\text { C6 N2 C10C9 } & -35.9(3) & \text { C36P2 } & \text { C32C35 } & 82.1(2)\end{array}$ 
Table 7 Hydrogen Atom Coordinates $\left(\AA \times 10^{4}\right)$ and Isotropic Displacement Parameters $\left(\AA^{2} \times 10^{3}\right)$

Atom

$\mathrm{H} 1$

$\mathrm{H} 3$

$\mathrm{H} 2$

$\mathrm{H} 3 \mathrm{~A}$

H1A

$\mathrm{H} 2 \mathrm{~A}$

$\mathrm{H} 3 \mathrm{~B}$

$\mathrm{H} 4$

H6

H7A

H7B

H8A

H8B

H9A

H9B

H11

H13A

H13B

H13C

H14A

H14B

H14C

H15A

H15B

H15C

H17A

H17B

H17C

H18A

H18B

H18C

H19A

H19B

H19C

$\mathrm{H} 21 \mathrm{~A}$

H21B

$\mathrm{H} 22 \mathrm{~A}$

H22B

$\mathrm{H} 23 \mathrm{~A}$

$\mathrm{H} 23 \mathrm{~B}$

$\mathrm{H} 24 \mathrm{~A}$

H24B

H25

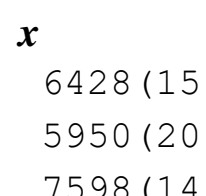

$7598(14)$

$7406(16)$

3633

2745

3333

4810

6300

7026

6116

6509

5630

6567

7319

6297

4293

3455

3815

5022

4073

4890

4071

3501

4406

7411

7287

7583

6401

6078

5422

5646

6377

6641

7804

8556

8456

9147

9754

9821

9309

8557

8692

\section{$y$}

5547 (14)

$6528(15)$

$7193(14)$

6408 (12)

6390

7278

8188

8181

7399

7736

8109

7640

7307

6443

6725

5377

5891

5428

5631

4275

4548

5028

4502

4299

3937

4784

4945

4215

3692

4405

3918

3676

4067

3500

7514

7417

6729

7297

6196

6602

5525

5591

6392 $z$

$6238(10)$

$5991(10)$

$5408(8)$

$6405(7)$

6336

6518

6902

7073

6593

7198

7235

7933

7758

7985

7673

7665

7354

7396

6917

7718

7822

7881

6631

7053

6965

7095

6580

6734

6333

6160

6411

7224

7494

7146

6534

6175

7008

6888

6826

6369

6203

6565

5788
$\mathrm{U}(\mathrm{eq})$

$38(8)$

$38(8)$

$28(7)$

$15(6)$

25

33

50

44

24

31

31

33

33

33

33

30

51

51

51

54

54

54

59

59

59

44

44

44

41

41

41

51

51

51

31

31

39

39

38

38

34

34

29 


\begin{tabular}{lllll} 
H27 & 8466 & 4782 & 5817 & 31 \\
H28 & 7654 & 4152 & 5307 & 34 \\
H29 & 6545 & 4665 & 4906 & 30 \\
H31A & 5381 & 5789 & 5137 & 25 \\
H31B & 5885 & 5807 & 4674 & 25 \\
H33A & 6543 & 4065 & 53 \\
H33B & 588 & 7093 & 3825 & 53 \\
H33C & 6466 & 7313 & 4079 & 53 \\
H34A & 5605 & 6660 & 4756 & 47 \\
H34B & 7740 & 6796 & 4234 & 47 \\
H34C & 7785 & 6182 & 4420 & 48 \\
H35A & 7233 & 8116 & 4551 & 48 \\
H35B & 6411 & 7930 & 4293 \\
H35C & 7281 & 7879 & 4822 & 42 \\
H37A & 7244 & 6825 & 4367 & 42 \\
H37B & 4561 & 6938 & 4671 & 42 \\
H37C & 3727 & 6290 & 4740 & 40 \\
H38A & 4316 & 6578 & 5543 & 40 \\
H38B & 4493 & 7199 & 5456 & 40 \\
H38C & 3866 & 5681 & 42 \\
H39A & 4789 & 5107 & 42 \\
H39B & 5231 & 4935 & 42 \\
H39C & 4258 & 8135 & 4597 & 4 \\
\hline
\end{tabular}

Table 8 Solvent masks information

$\begin{array}{lccccc}\text { Number } & \text { X } & \text { Y } & \text { Z } & \text { Volume } & \begin{array}{c}\text { Electron } \\ \text { count }\end{array} \text { Content } \\ 1 & 0.000 & 0.000 & 0.000 & 388 & 182 \\ 2 & 0.000 & 0.500 & 0.500 & 388 & 182 \\ 3 & 0.500 & 0.500 & 0.000 & 388 & 182 \\ 4 & 0.500 & 1.000 & 0.500 & 388 & 182\end{array}$


Western Univeristy Sample Code b20077

X-ray Structure Report for complex 6 crystallized from hexane/benzene solvent mixture, CCDC \#2008084

\author{
Prepared by \\ Louise N. Dawe, PhD \\ Department of Chemistry and Biochemistry \\ Wilfrid Laurier University \\ Science Building \\ 75 University Ave. W. \\ Waterloo, ON, ON \\ ldawe@wlu.ca
}

February 25, 2020

Introduction

Dr. Paul D. Boyle, Department of Chemistry X-Ray Facility, Western University, is acknowledged for assistance with X-ray data collection.

$\mathrm{H}$-atoms, except $\mathrm{H} 1, \mathrm{H} 2 \mathrm{H} 3$, and $\mathrm{H} 3 \mathrm{~A}$, were introduced in calculated positions and refined on a riding model. All other atoms were introduced in difference map positions and refined isotropically $(\mathrm{H} 1, \mathrm{H} 2 \mathrm{H} 3$, and $\mathrm{H} 3 \mathrm{~A})$ or anisotropically (all non-hydrogen atoms.)

A disordered lattice solvent benzene is present in the asymmetric unit. This has been split and modeled with enhanced rigid bond, same distance, and flat plane restraints (SHELX RIGU, SADI, and FLAT; all with default standard uncertainties).

$\mathrm{H} 1$ and $\mathrm{H} 2$ are singly-bonded Ru-bound hydrides. Distance restraints with default standard uncertainty (SHELX DFIX), and a target distance based on the average singly bonded $\mathrm{Ru}-\mathrm{H}$ bond from a survey of the Cambridge Structural Database were employed for Ru1-H1 and Ru2-H2 bond lengths. A distance restraint was also used for the N3-H3A bond (where H3A was introduced from its difference map position and was allowed to refine positionally and

isotropically). 


\section{Experimental}

A single crystal of $\mathrm{C}_{46} \mathrm{H}_{72} \mathrm{~N}_{4} \mathrm{O}_{2} \mathrm{P}_{2} \mathrm{Ru}_{2}$ was selected and collected on a Bruker APEX-II CCD diffractometer. The crystal was kept at 110(2) K during data collection. Using Olex2 [1], the structure was solved with the ShelXT [2] structure solution program using Direct Methods and refined with the ShelXL [3] refinement package using Least Squares minimisation.

\section{Crystal structure determination}

Crystal Data for $\mathrm{C}_{46} \mathrm{H}_{72} \mathrm{~N}_{4} \mathrm{O}_{2} \mathrm{P}_{2} \mathrm{Ru}_{2}(M=977.15 \mathrm{~g} / \mathrm{mol})$ ): orthorhombic, space group Pbca (no. 61), $a=$ 15.2483(2) $\AA, b=19.9605(2) \AA, c=30.3279(3) \AA, V=9230.71(18) \AA^{3}, Z=8, T=110(2) \mathrm{K}, \mu(\mathrm{Mo} \mathrm{K \alpha})=0.764$ $\mathrm{mm}^{-1}$, Dcalc $=1.406 \mathrm{~g} / \mathrm{cm}^{3}, 166294$ reflections measured $\left(4.886^{\circ} \leq 2 \theta \leq 51.358^{\circ}\right), 8760$ unique $(8756$ with I > $\left.2 \sigma(\mathrm{I}) ; R_{\text {int }}=0.0624, \mathrm{R}_{\text {sigma }}=0.0178\right)$ which were used in all calculations. The final $R_{1}$ was $0.0559(\mathrm{I}>2 \sigma(\mathrm{I}))$ and $w R_{2}$ was 0.1038 (all data). 
Table 1 Crystal data and structure refinement.

Identification code

Empirical formula

Formula weight

Temperature/K

Crystal system

Space group

$\mathrm{a} / \AA$

$\mathrm{b} / \AA$

$\mathrm{c} / \AA ̊ \AA$

$\alpha /^{\circ}$

$\beta /{ }^{\circ}$

$\gamma /{ }^{\circ}$

Volume $/ \AA^{3}$

Z

$\rho_{\text {calcg }} / \mathrm{cm}^{3}$

$\mu / \mathrm{mm}^{-1}$

$\mathrm{F}(000)$

Crystal size $/ \mathrm{mm}^{3}$

Radiation b20077 (Western Xray Code)

$\mathrm{C}_{46} \mathrm{H}_{72} \mathrm{~N}_{4} \mathrm{O}_{2} \mathrm{P}_{2} \mathrm{Ru}_{2}$

977.15

110(2)

orthorhombic

Pbca

15.2483(2)

$19.9605(2)$

$30.3279(3)$

90

90

90

9230.71(18)

8

1.406

0.764

4080.0

$0.297 \times 0.283 \times 0.112$

$\operatorname{MoK} \alpha(\lambda=0.71073)$

$2 \Theta$ range for data collection $/{ }^{\circ} 4.886$ to 51.358

Index ranges

$-18 \leq \mathrm{h} \leq 18,-24 \leq \mathrm{k} \leq 24,-36 \leq 1 \leq 36$

Reflections collected

166294

Independent reflections

Data/restraints/parameters

8760 [8756 with $\mathrm{I}>2 \sigma(\mathrm{I}) ; \mathrm{R}_{\text {int }}=0.0624, \mathrm{R}_{\text {sigma }}=0.0178$ ]

$8760 / 144 / 588$

Goodness-of-fit on $\mathrm{F}^{2}$

1.507

Final $\mathrm{R}$ indexes $[\mathrm{I}>=2 \sigma(\mathrm{I})] \quad \mathrm{R}_{1}=0.0559, \mathrm{wR}_{2}=0.1038$

Final $\mathrm{R}$ indexes [all data] $\quad \mathrm{R}_{1}=0.0559, \mathrm{wR}_{2}=0.1038$

Largest diff. peak/hole / e $\AA^{-3} 0.48 /-0.61$ 
Table 2 Fractional Atomic Coordinates $\left(\times 10^{4}\right)$ and Equivalent Isotropic Displacement Parameters $\left(\AA^{2} \times 10^{3}\right)$. Ueq is defined as $1 / 3$ of of the trace of the orthogonalised UIJ tensor.

\begin{tabular}{|c|c|c|c|c|}
\hline Atom & $x$ & $y$ & $z$ & $\mathbf{U}(\mathbf{e q})$ \\
\hline Ru1 & $5623.7(2)$ & $5947.9(2)$ & $3606.4(2)$ & $11.14(9)$ \\
\hline $\mathrm{Ru} 2$ & $6850.3(2)$ & $6899.6(2)$ & $4328.3(2)$ & $11.82(9)$ \\
\hline P1 & $5570.5(8)$ & $5144.6(6)$ & $3063.0(4)$ & $13.9(2)$ \\
\hline $\mathrm{P} 2$ & $6073.8(7)$ & $6839.5(6)$ & $4965.2(4)$ & $12.4(2)$ \\
\hline $\mathrm{O} 1$ & $4476(3)$ & $5171.0(18)$ & $4220.8(12)$ & $30.1(9)$ \\
\hline $\mathrm{O} 2$ & $6399(2)$ & $8330.0(17)$ & $4134.3(12)$ & $26.6(8)$ \\
\hline N1 & $4693(2)$ & $6723.7(17)$ & $3414.8(12)$ & $11.6(7)$ \\
\hline $\mathrm{N} 2$ & $6293(2)$ & $6495.4(18)$ & $3102.5(12)$ & $13.6(8)$ \\
\hline N3 & $7757(3)$ & $6620(2)$ & $3810.5(13)$ & $17.5(8)$ \\
\hline N4 & $7191(2)$ & $5914.6(19)$ & $4504.6(12)$ & $13.1(8)$ \\
\hline $\mathrm{C} 1$ & $3838(3)$ & $6748(2)$ & $3524.1(14)$ & $14.9(9)$ \\
\hline $\mathrm{C} 2$ & $3290(3)$ & $7263(2)$ & $3400.0(15)$ & $19.1(10)$ \\
\hline $\mathrm{C} 3$ & $3635(3)$ & $7782(3)$ & $3157.3(17)$ & $25.4(11)$ \\
\hline $\mathrm{C} 4$ & $4523(3)$ & $7779(2)$ & $3056.6(16)$ & $22.9(11)$ \\
\hline $\mathrm{C} 5$ & $5039(3)$ & $7244(2)$ & $3189.3(15)$ & $15.7(9)$ \\
\hline C6 & $6033(3)$ & $7208(2)$ & $3130.3(15)$ & $16.8(10)$ \\
\hline $\mathrm{C} 7$ & $6406(3)$ & $7641(2)$ & $2755.6(16)$ & $21.1(10)$ \\
\hline $\mathrm{C} 8$ & $6251(3)$ & $7323(3)$ & $2307.5(16)$ & $23.5(11)$ \\
\hline C9 & $6667(3)$ & $6634(2)$ & $2307.7(15)$ & $19.7(10)$ \\
\hline $\mathrm{C} 10$ & $6332(3)$ & $6211(2)$ & $2689.7(15)$ & $17.2(10)$ \\
\hline C11 & $6113(3)$ & $5559(2)$ & $2623.0(15)$ & $17.2(10)$ \\
\hline $\mathrm{C} 12$ & $4481(3)$ & $4875(2)$ & $2813.0(15)$ & $19.1(10)$ \\
\hline $\mathrm{C} 13$ & 3911 (3) & $5506(3)$ & $2801.5(18)$ & $26.2(12)$ \\
\hline $\mathrm{C} 14$ & $4553(4)$ & $4624(3)$ & $2337.8(16)$ & $25.6(11)$ \\
\hline $\mathrm{C} 15$ & $4004(4)$ & $4354(3)$ & $3102.0(19)$ & $29.5(12)$ \\
\hline C16 & $6247(3)$ & $4363(2)$ & $3178.4(16)$ & $17.7(10)$ \\
\hline $\mathrm{C} 17$ & $6172(4)$ & $3820(2)$ & $2821.0(17)$ & $25.9(11)$ \\
\hline C18 & $5989(3)$ & $4054(2)$ & $3625.0(16)$ & $22.1(10)$ \\
\hline C19 & $7207(3)$ & $4575(3)$ & $3203.0(17)$ & $24.4(11)$ \\
\hline $\mathrm{C} 20$ & $4952(3)$ & $5477(2)$ & $3991.2(15)$ & $16.8(10)$ \\
\hline $\mathrm{C} 21$ & $8258(3)$ & $7152(2)$ & $3585.5(16)$ & $19.7(10)$ \\
\hline $\mathrm{C} 22$ & $8853(4)$ & $6886(3)$ & $3228.7(18)$ & $28.3(12)$ \\
\hline $\mathrm{C} 23$ & $9468(3)$ & $6356(3)$ & $3407.3(18)$ & $26.5(11)$ \\
\hline $\mathrm{C} 24$ & $8970(3)$ & $5811(3)$ & $3654.2(18)$ & $24.6(11)$ \\
\hline $\mathrm{C} 25$ & $8363(3)$ & $6113(2)$ & $3995.8(16)$ & $17.5(10)$ \\
\hline $\mathrm{C} 26$ & $7863(3)$ & $5628(2)$ & $4281.9(15)$ & $17.3(10)$ \\
\hline $\mathrm{C} 27$ & $8049(3)$ & $4955(2)$ & $4333.6(17)$ & $24.2(11)$ \\
\hline $\mathrm{C} 28$ & $7542(3)$ & $4582(2)$ & $4627.2(16)$ & $21.3(10)$ \\
\hline $\mathrm{C} 29$ & $6883(3)$ & $4882(2)$ & $4865.9(16)$ & $21.2(10)$ \\
\hline $\mathrm{C} 30$ & $6705(3)$ & $5560(2)$ & $4790.8(15)$ & $16.6(10)$ \\
\hline C31 & $5955(3)$ & $5917(2)$ & $5005.8(15)$ & $17.4(9)$ \\
\hline $\mathrm{C} 32$ & $6680(3)$ & $7068(2)$ & $5488.2(15)$ & $16.9(10)$ \\
\hline
\end{tabular}




\begin{tabular}{|c|c|c|c|c|}
\hline $\mathrm{C} 33$ & $7496(3)$ & $6612(3)$ & $5514.9(15)$ & $20.6(10)$ \\
\hline $\mathrm{C} 34$ & $6159(3)$ & $6968(3)$ & $5913.3(15)$ & $24.6(11)$ \\
\hline $\mathrm{C} 35$ & $6997(3)$ & $7799(2)$ & $5458.0(16)$ & $21.5(10)$ \\
\hline $\mathrm{C} 36$ & $4913(3)$ & $7159(2)$ & $5003.0(15)$ & $15.1(9)$ \\
\hline $\mathrm{C} 37$ & $4340(3)$ & $6780(2)$ & $5336.7(16)$ & $21.8(10)$ \\
\hline C38 & $4909(3)$ & $7909(2)$ & $5114.5(16)$ & $20.9(10)$ \\
\hline C39 & $4500(3)$ & 7077 (3) & $4546.4(15)$ & $20.0(10)$ \\
\hline $\mathrm{C} 40$ & $6579(3)$ & $7768(2)$ & $4202.8(15)$ & $17.8(10)$ \\
\hline $\mathrm{C} 41$ & $9386(10)$ & $5743(7)$ & $6139(6)$ & $36(3)$ \\
\hline $\mathrm{C} 42$ & $9510(8)$ & $5543(8)$ & $5709(5)$ & $33(3)$ \\
\hline $\mathrm{C} 43$ & $9013(9)$ & $5022(10)$ & $5537(4)$ & $42(3)$ \\
\hline $\mathrm{C} 44$ & $8398(11)$ & $4711(8)$ & $5806(5)$ & $52(4)$ \\
\hline $\mathrm{C} 45$ & $8288(13)$ & $4890(9)$ & $6241(5)$ & $46(4)$ \\
\hline $\mathrm{C} 46$ & $8769(12)$ & $5422(9)$ & $6398(5)$ & $43(4)$ \\
\hline C41A & $8950(20)$ & 5535 (17) & $6342(11)$ & $46(6)$ \\
\hline $\mathrm{C} 42 \mathrm{~A}$ & $9441(18)$ & $5718(13)$ & $5980(13)$ & $43(5)$ \\
\hline $\mathrm{C} 43 \mathrm{~A}$ & $9430(20)$ & $5340(16)$ & $5601(11)$ & $49(6)$ \\
\hline $\mathrm{C} 44 \mathrm{~A}$ & $8940(20)$ & $4759(18)$ & $5602(10)$ & $65(8)$ \\
\hline $\mathrm{C} 45 \mathrm{~A}$ & $8410(30)$ & $4580(17)$ & $5951(13)$ & $74(11)$ \\
\hline $\mathrm{C} 46 \mathrm{~A}$ & $8410(30)$ & 4985 (18) & $6317(12)$ & $56(8)$ \\
\hline
\end{tabular}


Table 3 Anisotropic Displacement Parameters $\left(\AA^{2} \times 10^{3}\right)$. The Anisotropic displacement factor exponent takes the form: $-2 \pi^{2}\left[h^{2} a^{* 2} U_{11}+2 h k a * b^{*} U_{12}+\ldots\right]$.

\begin{tabular}{|c|c|c|c|c|c|c|}
\hline Atom & $\mathbf{U}_{11}$ & $\mathbf{U}_{22}$ & $\mathbf{U}_{33}$ & $\mathbf{U}_{23}$ & $\mathbf{U}_{13}$ & $\mathbf{U}_{12}$ \\
\hline Ru1 & $13.64(17)$ & $9.33(16)$ & $10.45(16)$ & $1.60(13)$ & $0.74(14)$ & $-0.85(14)$ \\
\hline $\mathrm{Ru} 2$ & $11.93(17)$ & $11.08(17)$ & $12.45(17)$ & $1.68(14)$ & $-0.38(14)$ & $-2.03(14)$ \\
\hline $\mathrm{P} 1$ & $18.3(6)$ & $9.8(5)$ & $13.5(5)$ & $0.6(4)$ & $0.9(5)$ & $0.0(5)$ \\
\hline $\mathrm{P} 2$ & $12.8(5)$ & $12.7(5)$ & $11.8(5)$ & $0.8(4)$ & $-0.8(4)$ & $-1.3(4)$ \\
\hline $\mathrm{O} 1$ & $40(2)$ & $26(2)$ & 24.1 (19) & $5.4(16)$ & $13.2(17)$ & $-11.7(17)$ \\
\hline $\mathrm{O} 2$ & $37(2)$ & $13.2(17)$ & $29(2)$ & $2.8(15)$ & $-3.0(17)$ & $0.3(15)$ \\
\hline N1 & $14.9(18)$ & $7.8(17)$ & $12.2(18)$ & $-1.8(14)$ & $-2.6(15)$ & $1.4(14)$ \\
\hline $\mathrm{N} 2$ & $17.2(19)$ & $12.3(18)$ & $11.2(18)$ & $2.2(15)$ & $2.1(15)$ & $-1.8(15)$ \\
\hline N3 & $16.1(19)$ & $17(2)$ & $19(2)$ & $6.6(16)$ & $2.1(16)$ & $-0.7(16)$ \\
\hline N4 & $11.3(17)$ & $15.9(19)$ & $12.1(18)$ & $0.7(15)$ & $-1.4(14)$ & $0.4(15)$ \\
\hline $\mathrm{C} 1$ & $17(2)$ & $16(2)$ & $11(2)$ & $-4.5(17)$ & $-0.2(17)$ & $-1.6(18)$ \\
\hline $\mathrm{C} 2$ & $16(2)$ & $23(2)$ & $18(2)$ & $-6(2)$ & $-2.9(19)$ & $5(2)$ \\
\hline $\mathrm{C} 3$ & $25(3)$ & $22(3)$ & $29(3)$ & $3(2)$ & $-3(2)$ & $10(2)$ \\
\hline $\mathrm{C} 4$ & $31(3)$ & $14(2)$ & $23(3)$ & $6.2(19)$ & $0(2)$ & $3(2)$ \\
\hline $\mathrm{C} 5$ & $21(2)$ & $11(2)$ & $14(2)$ & $-1.9(18)$ & $-2.8(18)$ & $1.6(18)$ \\
\hline C6 & $18(2)$ & $16(2)$ & $17(2)$ & $3.5(19)$ & $-0.7(19)$ & $-1.2(19)$ \\
\hline $\mathrm{C} 7$ & $23(3)$ & $14(2)$ & $26(3)$ & $6(2)$ & $4(2)$ & $-2(2)$ \\
\hline $\mathrm{C} 8$ & $25(3)$ & $29(3)$ & $16(2)$ & $7(2)$ & $2(2)$ & $-2(2)$ \\
\hline C9 & $23(3)$ & $21(2)$ & $15(2)$ & $2.1(19)$ & $5.5(19)$ & $-3(2)$ \\
\hline C10 & $16(2)$ & $19(2)$ & $16(2)$ & $1.4(19)$ & 1.5 (19) & $-1.5(19)$ \\
\hline C11 & $28(3)$ & $14(2)$ & $9(2)$ & $0.3(17)$ & $1.3(19)$ & $2.9(19)$ \\
\hline C12 & $24(3)$ & $15(2)$ & $18(2)$ & $-5.0(19)$ & $-6(2)$ & $-3(2)$ \\
\hline C13 & $24(3)$ & $25(3)$ & $30(3)$ & $-13(2)$ & $-10(2)$ & $2(2)$ \\
\hline C14 & $31(3)$ & $23(3)$ & $23(3)$ & $-8(2)$ & $-9(2)$ & $0(2)$ \\
\hline C15 & $25(3)$ & $29(3)$ & $34(3)$ & $-2(2)$ & $-2(2)$ & $-10(2)$ \\
\hline C16 & $22(2)$ & $10(2)$ & $21(2)$ & $-1.9(18)$ & $-3(2)$ & $5.5(19)$ \\
\hline C17 & $37(3)$ & $18(2)$ & $23(3)$ & $-3(2)$ & $-6(2)$ & $6(2)$ \\
\hline C18 & $30(3)$ & $15(2)$ & $21(2)$ & $4(2)$ & $1(2)$ & $-2(2)$ \\
\hline C19 & $19(2)$ & $24(3)$ & $30(3)$ & $0(2)$ & $3(2)$ & $9(2)$ \\
\hline $\mathrm{C} 20$ & $23(2)$ & $14(2)$ & $14(2)$ & $-2.7(18)$ & $-0.8(19)$ & $0.0(19)$ \\
\hline C21 & $19(2)$ & $16(2)$ & $24(2)$ & $5(2)$ & $6(2)$ & $-3.9(19)$ \\
\hline $\mathrm{C} 22$ & $27(3)$ & $24(3)$ & $34(3)$ & $6(2)$ & $9(2)$ & $-2(2)$ \\
\hline $\mathrm{C} 23$ & $23(3)$ & $29(3)$ & $27(3)$ & $1(2)$ & $7(2)$ & $1(2)$ \\
\hline $\mathrm{C} 24$ & $17(2)$ & $26(3)$ & $31(3)$ & $4(2)$ & $8(2)$ & $1(2)$ \\
\hline $\mathrm{C} 25$ & $14(2)$ & $17(2)$ & $22(2)$ & $3.9(19)$ & $-1.8(19)$ & $-1.0(18)$ \\
\hline $\mathrm{C} 26$ & $17(2)$ & $23(2)$ & $13(2)$ & $1.8(19)$ & $-1.2(18)$ & $2.8(19)$ \\
\hline $\mathrm{C} 27$ & $24(3)$ & $21(2)$ & $27(3)$ & $3(2)$ & $8(2)$ & $6(2)$ \\
\hline C28 & $26(3)$ & $14(2)$ & $24(2)$ & $4.1(19)$ & $-1(2)$ & $4(2)$ \\
\hline $\mathrm{C} 29$ & $24(3)$ & $18(2)$ & $22(2)$ & $5.4(19)$ & $0(2)$ & $-4(2)$ \\
\hline $\mathrm{C} 30$ & $17(2)$ & $19(2)$ & $14(2)$ & $2.1(18)$ & $-0.2(18)$ & $-1.9(19)$ \\
\hline C31 & $15(2)$ & $19(2)$ & $18(2)$ & $2.5(19)$ & $2.1(18)$ & $-0.2(19)$ \\
\hline C32 & $15(2)$ & $22(2)$ & $13(2)$ & $1.5(18)$ & $-4.0(18)$ & $-3.1(19)$ \\
\hline
\end{tabular}




$\begin{array}{lrrrrrr}\text { C33 } & 16(2) & 27(3) & 18(2) & 7(2) & -7(2) & -3(2) \\ \text { C34 } & 23(3) & 39(3) & 12(2) & 3(2) & -2.4(19) & -9(2) \\ \text { C35 } & 18(2) & 27(3) & 19(2) & -6(2) & -6.2(19) & -4(2) \\ \text { C36 } & 15(2) & 18(2) & 13(2) & -1.8(18) & -2.9(17) & 0.4(18) \\ \text { C37 } & 15(2) & 24(3) & 27(3) & -1(2) & 4(2) & 0(2) \\ \text { C38 } & 19(2) & 21(2) & 23(3) & 0(2) & -1(2) & -2(2) \\ \text { C39 } & 12(2) & 29(3) & 19(2) & -6(2) & -6.2(18) & 6(2) \\ \text { C40 } & 19(2) & 20(3) & 14(2) & -1.4(19) & -2.0(18) & -1.6(19) \\ \text { C41 } & 50(6) & 32(5) & 25(6) & -3(4) & -9(5) & 1(4) \\ \text { C42 } & 38(5) & 35(6) & 26(6) & -3(4) & -11(4) & 7(5) \\ \text { C43 } & 53(6) & 37(7) & 34(5) & -8(4) & -13(4) & -1(5) \\ \text { C44 } & 65(7) & 48(7) & 45(6) & -7(5) & -11(5) & -11(5) \\ \text { C45 } & 59(7) & 38(7) & 41(6) & -3(4) & -12(5) & -5(6) \\ \text { C46 } & 61(8) & 40(7) & 28(5) & -3(4) & -5(5) & -8(6) \\ \text { C41A } & 53(14) & 36(10) & 48(9) & -4(7) & -10(7) & 0(8) \\ \text { C42A } & 49(10) & 31(8) & 48(11) & -3(7) & -11(9) & 5(7) \\ \text { C43A } & 62(13) & 34(11) & 49(9) & -5(6) & -6(9) & -2(8) \\ \text { C44A } & 95(16) & 48(12) & 52(9) & -12(8) & 5(9) & -23(11) \\ \text { C45A } & 108(19) & 60(11) & 54(10) & -18(10) & 11(12) & -37(14) \\ \text { C46A } & 73(16) & 47(11) & 47(9) & -8(7) & -5(10) & -15(10)\end{array}$




\section{Table 4 Bond Lengths}

Atom Atom Length/ $\AA$ Atom Atom Length $/ \AA$

\begin{tabular}{|c|c|c|c|c|c|}
\hline u1 & P1 & $.3009(12)$ & $\mathrm{C} 12$ & $\mathrm{C} 13$ & 1 \\
\hline u1 & N1 & $2.179(4)$ & $\mathrm{C} 12$ & $\mathrm{C} 14$ & $1.530(6)$ \\
\hline u1 & $\mathrm{N} 2$ & $2.138(4)$ & $\mathrm{C} 12$ & $\mathrm{C} 15$ & $1.542(7)$ \\
\hline u1 & $\mathrm{C} 20$ & $1.815(5)$ & $\mathrm{C} 16$ & $\mathrm{C} 17$ & $1.537(6)$ \\
\hline u2 & $\mathrm{P} 2$ & $2.2690(12)$ & $\mathrm{C} 16$ & $\mathrm{C} 18$ & $1.540(7)$ \\
\hline u2 & N3 & $2.165(4)$ & C16 & C19 & $1.525(7)$ \\
\hline u2 & N4 & $2.103(4)$ & $\mathrm{C} 21$ & $\mathrm{C} 22$ & $1.508(7)$ \\
\hline u2 & $\mathrm{C} 40$ & $1.821(5)$ & $\mathrm{C} 22$ & $\mathrm{C} 23$ & $1.515(7)$ \\
\hline 1 & $\mathrm{C} 11$ & $1.774(5)$ & $\mathrm{C} 23$ & $\mathrm{C} 24$ & $1.524(7)$ \\
\hline 1 & $\mathrm{C} 12$ & $1.904(5)$ & $\mathrm{C} 24$ & $\mathrm{C} 25$ & $1.515(7)$ \\
\hline 1 & $\mathrm{C} 16$ & $1.902(5)$ & $\mathrm{C} 25$ & $\mathrm{C} 26$ & $1.507(6)$ \\
\hline 2 & $\mathrm{C} 31$ & $1.855(5)$ & $\mathrm{C} 26$ & $\mathrm{C} 27$ & $1.383(7)$ \\
\hline 2 & C32 & $1.892(5)$ & $\mathrm{C} 27$ & $\mathrm{C} 28$ & $1.394(7)$ \\
\hline 2 & C36 & $1.885(5)$ & $\mathrm{C} 28$ & $\mathrm{C} 29$ & $1.376(7)$ \\
\hline 1 & $\mathrm{C} 20$ & $1.177(6)$ & $\mathrm{C} 29$ & C30 & $1.399(7)$ \\
\hline 2 & $\mathrm{C} 40$ & $1.174(6)$ & C30 & C31 & $1.496(6$ \\
\hline N1 & $\mathrm{C} 1$ & $1.346(6)$ & C32 & C33 & $1.544(7$ \\
\hline N1 & $\mathrm{C} 5$ & $1.350(6)$ & C32 & C34 & $1.528(6)$ \\
\hline $\mathrm{N} 2$ & C6 & $1.480(6)$ & $\mathrm{C} 32$ & $\mathrm{C} 35$ & $1.540(7)$ \\
\hline $\mathrm{N} 2$ & $\mathrm{C} 10$ & $1.376(6)$ & C36 & C37 & $1.536(6)$ \\
\hline N3 & $\mathrm{C} 21$ & $1.474(6)$ & C36 & C38 & $1.535(6)$ \\
\hline N3 & $\mathrm{C} 25$ & $1.482(6)$ & C36 & C39 & $1.530(6)$ \\
\hline 4 & $\mathrm{C} 26$ & $1.354(6)$ & C41 & $\mathrm{C} 42$ & $.377(10)$ \\
\hline J4 & $\mathrm{C} 30$ & $1.343(6)$ & $\mathrm{C} 41$ & C46 & $.383(11)$ \\
\hline 1 & $\mathrm{C} 2$ & $1.378(6)$ & $\mathrm{C} 42$ & $\mathrm{C} 43$ & $1.389(11)$ \\
\hline 22 & $\mathrm{C} 3$ & $1.376(7)$ & $\mathrm{C} 43$ & C44 & $1.390(11)$ \\
\hline 3 & $\mathrm{C} 4$ & $1.387(7)$ & C44 & $\mathrm{C} 45$ & $1.378(11)$ \\
\hline 4 & C5 & $1.387(6)$ & $\mathrm{C} 45$ & $\mathrm{C} 46$ & $1.374(11)$ \\
\hline $\mathrm{c} 5$ & C6 & $1.528(6)$ & C41 & $\mathrm{C} 42 \mathrm{~F}$ & $1.377(14)$ \\
\hline C6 & C7 & $1.536(6)$ & $\mathrm{C} 41 \mathrm{~A}$ & AC46A & $1.375(14$ \\
\hline $\mathrm{C} 7$ & $\mathrm{C} 8$ & $1.518(7)$ & $\mathrm{C} 42 \mathrm{~A}$ & C $433 \mathrm{~A}$ & $1.376(14$ \\
\hline C8 & C9 & $1.513(7)$ & $\mathrm{C} 43 \mathrm{~A}$ & C $44 \mathrm{~A}$ & $1.379(14$ \\
\hline C9 & $\mathrm{C} 10$ & $1.522(6)$ & C44 & AC45A & $1.379(14)$ \\
\hline C10 & $\mathrm{C} 11$ & $1.359(6)$ & $\mathrm{C} 45 \mathrm{~A}$ & A C46A & $1.373(14$ \\
\hline
\end{tabular}


Table 5 Bond Angles

\section{Atom Atom Atom}

N1 Ru1 P1

N2 Ru1 P1

N2 Ru1 N1

C20 Ru1 P1

C20 Ru1 N1

C20 Ru1 N2

N3 Ru2 P2

N4 Ru2 P2

N4 Ru2 N3

C40 Ru2 P2

C40 Ru2 N3

C40 Ru2 N4

C11 P1 Ru1

C11 P1 C12

C11 P1 C16

C12 P1 Ru1

C16 P1 Ru1

C16 P1 C12

C31 P2 Ru2

C31 P2 C32

C31 P2 C36

C32 P2 Ru2

C36 P2 Ru2

C36 P2 C32

C1 N1 Ru1

C1 N1 C5

C5 N1 Ru1

C6 N2 Ru1

C10 N2 Ru1

C10 N2 C6

C21 N3 Ru2

C21 N3 C25

C25 N3 Ru2

C26 N4 Ru2

C30 N4 Ru2

C30 N4 C26

N1 C1 C2

$\mathrm{C} 3 \quad \mathrm{C} 2 \mathrm{C} 1$

$\mathrm{C} 2 \mathrm{C} 3 \quad \mathrm{C} 4$

C3 C4 C5

N1 C5 C4

N1 C5 C6

C4 C5 C6

\section{Angle $/^{\circ} \quad$ Atom Atom Atom}

106.33(10) C13 C12 C15

82.01(10) C14 C12 P1

75.95(14) C14 C12 C13

94.58(14) C14 C12 C15

99.88(18) C15 C12 P1

$173.52(18) \quad \mathrm{C} 17 \quad \mathrm{C} 16 \quad \mathrm{P} 1$

159.56(11) C17 C16 C18

$82.12(10)$ C18 C16 P1

$77.65(14)$ C19 C16 P1

$96.30(15) \quad \mathrm{C} 19 \quad \mathrm{C} 16 \quad \mathrm{C} 17$

103.79(18) C19 C16 C18

177.01(18) O1 C20 Ru1

$101.40(16)$ N3 C21 C22

103.8(2) C21 C22 C23

105.5(2) $\quad$ C22 $\quad$ C23 $\quad$ C24

120.85(15) C25 C24 C23

$114.86(15) \quad \mathrm{N} 3 \quad \mathrm{C} 25 \quad \mathrm{C} 24$

$108.4(2) \quad \mathrm{N} 3 \quad \mathrm{C} 25 \quad \mathrm{C} 26$

99.21(15) C26 C25 C24

$103.4(2) \quad \mathrm{N} 4 \quad \mathrm{C} 26 \quad \mathrm{C} 25$

$103.9(2) \quad \mathrm{N} 4 \quad \mathrm{C} 26 \quad \mathrm{C} 27$

116.47(15) C27 C26 C25

$121.60(15) \quad$ C26 C27 C28

109.1(2) C29 C28 C27

$126.2(3)$ C28 C29 C30

118.4(4) N4 C30 C29

$115.3(3) \quad \mathrm{N} 4 \quad \mathrm{C} 30 \quad \mathrm{C} 31$

108.8(3) $\mathrm{C} 29 \quad \mathrm{C} 30 \quad \mathrm{C} 31$

117.4(3) $\mathrm{C} 30 \quad \mathrm{C} 31 \quad \mathrm{P} 2$

117.4(4) C33 C32 P2

$118.8(3) \quad \mathrm{C} 34 \quad \mathrm{C} 32 \quad \mathrm{P} 2$

110.1(4) $\mathrm{C} 34 \quad \mathrm{C} 32 \quad \mathrm{C} 33$

107.4(3) C34 C32 C35

$117.0(3)$ C35 C32 P2

121.4(3) C35 C32 C33

121.2 (4) C37 C36 P2

123.2 (4) C38 C36 P2

118.4(4) $\mathrm{C} 38 \quad \mathrm{C} 36 \quad \mathrm{C} 37$

$119.2(5)$ C39 C36 P2

119.5 (5) $\quad$ C39 $\quad$ C36 $\quad$ C37

121.2(4) C39 C36 C38

$114.3(4) \quad \mathrm{O} 2 \quad \mathrm{C} 40 \mathrm{Ru} 2$

124.4(4) C42 C41 C46
Angle/ ${ }^{\circ}$

$107.5(4)$

$113.8(4)$

$106.8(4)$

$110.4(4)$

$112.1(3)$

$114.1(3)$

$108.6(4)$

$110.6(3)$

$107.6(3)$

$107.5(4)$

$108.3(4)$

$175.8(4)$

$113.0(4)$

$111.2(4)$

$111.5(4)$

$110.8(4)$

$113.2(4)$

$110.0(4)$

$116.5(4)$

113.5 (4)

$120.6(4)$

$125.9(4)$

$118.5(5)$

$120.5(4)$

$118.6(4)$

$120.5(4)$

$116.9(4)$

$122.5(4)$

$111.6(3)$

$107.2(3)$

$114.9(3)$

$107.3(4)$

$109.8(4)$

$109.4(3)$

$108.1(4)$

114.1 (3)

$110.3(3)$

$109.5(4)$

$107.2(3)$

$108.0(4)$

$107.5(4)$

178.1 (4)

119.7 (12) 


$\begin{array}{lllllllr}\text { N2 } & \text { C6 } & \text { C5 } & 108.5(4) & \text { C41 } & \text { C42 } & \text { C43 } & 120.0(10) \\ \text { N2 } & \text { C6 } & \text { C7 } & 113.5(4) & \text { C42 } & \text { C43 } & \text { C44 } & 118.7(10) \\ \text { C5 } & \text { C6 } & \text { C7 } & 115.4(4) & \text { C45 } & \text { C44 } & \text { C43 } & 122.0(13) \\ \text { C8 } & \text { C7 } & \text { C6 } & 111.7(4) & \text { C46 } & \text { C45 } & \text { C44 } & 117.8(16) \\ \text { C9 } & \text { C8 } & \text { C7 } & 108.3(4) & \text { C45 } & \text { C46 } & \text { C41 } & 121.7(15) \\ \text { C8 } & \text { C9 } & \text { C10 } & 111.3(4) & \text { C46A C41AC42A } & 120(3) \\ \text { N2 } & \text { C10 } & \text { C9 } & 118.5(4) & \text { C43A C42A C41A } & 121(3) \\ \text { C11 } & \text { C10 } & \text { N2 } & 121.3(4) & \text { C42A C43AC44A } & 118(2) \\ \text { C11 } & \text { C10 } & \text { C9 } & 120.1(4) & \text { C43A C44AC45A } & 122(3) \\ \text { C10 } & \text { C11 } & \text { P1 } & 116.7(4) & \text { C46A C45AC44A } & 118(3) \\ \text { C13 } & \text { C12 } & \text { P1 } & 105.7(3) & \text { C45A C46AC41A } & 121(3)\end{array}$


Table 6 Torsion Angles

\begin{tabular}{|c|c|c|c|c|c|c|c|}
\hline B & D & Angle ${ }^{\circ}$ & $\mathbf{A}$ & B & $\mathbf{C}$ & D & Angle $/^{\circ}$ \\
\hline Ru1 P1 & $\mathrm{C} 11 \mathrm{C} 10$ & $-5.8(4)$ & $\mathrm{C} 10$ & $\mathrm{~N} 2$ & C6 & $\mathrm{C} 5$ & $91.5(5)$ \\
\hline Ru1 N1 & $\mathrm{C} 1 \mathrm{C} 2$ & $178.4(3)$ & $\mathrm{C} 10$ & $\mathrm{~N} 2$ & C6 & $\mathrm{C} 7$ & $-38.1(6)$ \\
\hline Ru1 N1 & $\mathrm{C} 5 \mathrm{C} 4$ & $-178.4(3)$ & C12 & $\mathrm{P} 1$ & $\mathrm{C} 11$ & $\mathrm{C} 10$ & $120.3(4)$ \\
\hline u1 N1 & $\mathrm{C} 5 \mathrm{C} 6$ & $-3.0(5)$ & $\mathrm{C} 16$ & P1 & $\mathrm{C} 11$ & $\mathrm{C} 10$ & $-125.9(4)$ \\
\hline Ru1 N2 & C6 C5 & $-44.9(4)$ & $\mathrm{C} 21$ & N3 & $\mathrm{C} 25$ & $\mathrm{C} 24$ & $-56.1(5)$ \\
\hline Ru1 N2 & C6 C7 & $-174.5(3)$ & $\mathrm{C} 21$ & N3 & $\mathrm{C} 25$ & $\mathrm{C} 26$ & $171.6(4)$ \\
\hline Ru1 N2 & $\mathrm{C} 10 \mathrm{C} 9$ & $170.0(3)$ & $\mathrm{C} 21$ & $\mathrm{C} 22$ & $\mathrm{C} 23$ & $\mathrm{C} 24$ & $52.1(6)$ \\
\hline Ru1 N2 & $\mathrm{C} 10 \mathrm{C} 11$ & $-12.8(6)$ & $\mathrm{C} 22$ & $\mathrm{C} 23$ & $\mathrm{C} 24$ & $\mathrm{C} 25$ & $-51.4(6)$ \\
\hline $\mathrm{Ru} 2 \mathrm{P} 2$ & C31 C30 & $28.9(3)$ & $\mathrm{C} 23$ & $\mathrm{C} 24$ & $\mathrm{C} 25$ & N3 & $54.1(6)$ \\
\hline $\mathrm{Ru} 2 \mathrm{P} 2$ & C32 C33 & $-56.2(3)$ & $\mathrm{C} 23$ & $\mathrm{C} 24$ & $\mathrm{C} 25$ & $\mathrm{C} 26$ & $-176.9(4)$ \\
\hline $\mathrm{Ru} 2 \mathrm{P} 2$ & C32 C34 & $-175.3(3)$ & C24 & $\mathrm{C} 25$ & $\mathrm{C} 26$ & N4 & $-165.3(4)$ \\
\hline $\mathrm{Ru} 2 \mathrm{P} 2$ & C32 C35 & $60.8(3)$ & $\mathrm{C} 24$ & $\mathrm{C} 25$ & $\mathrm{C} 26$ & $\mathrm{C} 27$ & $15.9(7)$ \\
\hline $\mathrm{Ru} 2 \mathrm{P} 2$ & C36C37 & $149.5(3)$ & $\mathrm{C} 25$ & N3 & $\mathrm{C} 21$ & $\mathrm{C} 22$ & $56.5(5)$ \\
\hline $\mathrm{Ru} 2 \mathrm{P} 2$ & C36 C38 & $-86.9(3)$ & $\mathrm{C} 25$ & $\mathrm{C} 26$ & $\mathrm{C} 27$ & $\mathrm{C} 28$ & $177.2(5)$ \\
\hline $\mathrm{Ru} 2 \mathrm{P} 2$ & C36C39 & $29.9(4)$ & C26 & N4 & $\mathrm{C} 30$ & $\mathrm{C} 29$ & $0.0(7)$ \\
\hline $\mathrm{Ru} 2 \mathrm{~N} 3$ & $\mathrm{C} 21 \mathrm{C} 22$ & $-179.1(3)$ & $\mathrm{C} 26$ & N4 & $\mathrm{C} 30$ & C31 & $-177.6(4)$ \\
\hline $\mathrm{Ru} 2 \mathrm{~N} 3$ & $\mathrm{C} 25 \mathrm{C} 24$ & $173.2(3)$ & $\mathrm{C} 26$ & $\mathrm{C} 27$ & $\mathrm{C} 28$ & $\mathrm{C} 29$ & $-1.2(8)$ \\
\hline $\mathrm{Ru} 2 \mathrm{~N} 3$ & $\mathrm{C} 25 \mathrm{C} 26$ & $40.9(4)$ & $\mathrm{C} 27$ & $\mathrm{C} 28$ & $\mathrm{C} 29$ & $\mathrm{C} 30$ & $3.2(8)$ \\
\hline Ru2N4 & C26C25 & $10.3(5)$ & C28 & C29 & $\mathrm{C} 30$ & N4 & $-2.7(7)$ \\
\hline $\mathrm{Ru} 2 \mathrm{~N} 4$ & $\mathrm{C} 26 \mathrm{C} 27$ & $-170.9(4)$ & $\mathrm{C} 28$ & $\mathrm{C} 29$ & $\mathrm{C} 30$ & $\mathrm{C} 31$ & $174.8(4)$ \\
\hline Ru2N4 & C $30 \mathrm{C} 29$ & $172.7(3)$ & $\mathrm{C} 29$ & C30 & $\mathrm{C} 31$ & P2 & $164.2(4)$ \\
\hline Ru2N4 & C 30 C 31 & $-4.9(5)$ & C30 & N4 & $\mathrm{C} 26$ & $\mathrm{C} 25$ & $-176.8(4)$ \\
\hline N1 C1 & $\mathrm{C} 2 \mathrm{C} 3$ & $-0.9(7)$ & C30 & $\mathrm{N} 4$ & $\mathrm{C} 26$ & $\mathrm{C} 27$ & $2.1(7)$ \\
\hline N1 C5 & C6 N2 & $31.6(5)$ & C31 & P2 & C32 & $\mathrm{C} 33$ & $51.4(3)$ \\
\hline $\mathrm{N} 1 \mathrm{C} 5$ & C6 C7 & $160.2(4)$ & C31 & P2 & C32 & C34 & $-67.7(4)$ \\
\hline N2 C6 & C7 C8 & $49.0(5)$ & C31 & P2 & C32 & C35 & $168.4(3)$ \\
\hline $\mathrm{N} 2 \mathrm{C} 1 \mathrm{C}$ & C11 P1 & $12.3(6)$ & C31 & P2 & C36 & C37 & $39.3(4)$ \\
\hline N3 C21 & I C22 C23 & $-55.3(6)$ & $\mathrm{C} 31$ & P2 & C36 & C38 & $162.9(3)$ \\
\hline N3 $\mathrm{C} 25$ & $5 \mathrm{C} 26 \mathrm{~N} 4$ & $-34.8(5)$ & C31 & P2 & C36 & C39 & $-80.3(3)$ \\
\hline N3 C25 & C26C27 & $146.5(5)$ & C32 & P2 & $\mathrm{C} 31$ & $\mathrm{C} 30$ & $-91.3(4)$ \\
\hline $\mathrm{N} 4 \mathrm{C} 2 \mathrm{C}$ & $5 \mathrm{C} 27 \mathrm{C} 28$ & $-1.4(7)$ & $\mathrm{C} 32$ & P2 & $\mathrm{C} 36$ & C37 & $-70.5(4)$ \\
\hline $\mathrm{N} 4 \mathrm{C} 3 \mathrm{C}$ & $\mathrm{C} 31 \mathrm{P} 2$ & $-18 \cdot 3(5)$ & $\mathrm{C} 32$ & P2 & $\mathrm{C} 36$ & $\mathrm{C} 38$ & $53.1(4)$ \\
\hline $\mathrm{C} 1 \mathrm{~N} 1$ & $\mathrm{C} 5 \mathrm{C} 4$ & $-2.6(6)$ & C32 & P2 & C36 & C39 & $170.0(3)$ \\
\hline C1 N1 & C5 C6 & $172.9(4)$ & C36 & $\mathrm{P} 2$ & $\mathrm{C} 31$ & $\mathrm{C} 30$ & $154.8(3)$ \\
\hline $\mathrm{C} 1 \mathrm{C} 2$ & $\mathrm{C} 3 \mathrm{C} 4$ & $-1.7(7)$ & $\mathrm{C} 36$ & P2 & $\mathrm{C} 32$ & $\mathrm{C} 33$ & $161.5(3)$ \\
\hline $\mathrm{C} 2 \mathrm{C} 3$ & $\mathrm{C} 4 \mathrm{C} 5$ & $2.1(8)$ & C36 & $\mathrm{P} 2$ & $\mathrm{C} 32$ & $\mathrm{C} 34$ & $42.4(4)$ \\
\hline $\mathrm{C} 3 \mathrm{C} 4$ & C5 N1 & $0.1(7)$ & C36 & $\mathrm{P} 2$ & $\mathrm{C} 32$ & $\mathrm{C} 35$ & $-81.6(4)$ \\
\hline C3 $\mathrm{C} 4$ & C5 $\mathrm{C} 6$ & $-174.9(5)$ & C41 & $\mathrm{C} 42$ & $\mathrm{C} 43$ & C44 & $-0.2(17)$ \\
\hline C4 C5 & C6 N2 & $-153.0(4)$ & C42 & C41 & $\mathrm{C} 46$ & C45 & $2.1(18)$ \\
\hline C4 C5 & C6 C7 & $-24.5(7)$ & C42 & $\mathrm{C} 43$ & C44 & $\mathrm{C} 45$ & $-2(2)$ \\
\hline C5 N1 & $\mathrm{C} 1 \mathrm{C} 2$ & $3.0(6)$ & C43 & C44 & $\mathrm{C} 45$ & C46 & $4(2)$ \\
\hline C5 C6 & C7 C8 & $-77.0(5)$ & C44 & $\mathrm{C} 45$ & $\mathrm{C} 46$ & C41 & $-4(2)$ \\
\hline C6 N2 & $\mathrm{C} 10 \mathrm{C} 9$ & $37.3(6)$ & C46 & $\mathrm{C} 41$ & $\mathrm{C} 42$ & C43 & $0.2(15)$ \\
\hline
\end{tabular}







Table 7 Hydrogen Atom Coordinates $\left(\AA \times 10^{4}\right)$ and Isotropic Displacement Parameters $\left(\AA^{2} \times 10^{3}\right)$

\section{Atom}

H1

$\mathrm{H} 3$

$\mathrm{H} 2$

$\mathrm{H} 3 \mathrm{~A}$

$\mathrm{H} 1 \mathrm{~A}$

$\mathrm{H} 2 \mathrm{~A}$

$\mathrm{H} 3 \mathrm{~B}$

$\mathrm{H} 4$

H6

H7A

H7B

H8A

H8B

H9A

H9B

H11

H13A

H13B

H13C

H14A

H14B

H14C

H15A

H15B

H15C

H17A

H17B

H17C

H18A

H18B

H18C

H19A

H19B

H19C

$\mathrm{H} 21 \mathrm{~A}$

H21B

$\mathrm{H} 22 \mathrm{~A}$

H22B

$\mathrm{H} 23 \mathrm{~A}$

H23B

$\mathrm{H} 24 \mathrm{~A}$

H24B

H 25

$x \quad y$

$6420(20)$

$5950(40)$

$7670(30)$

$7420(30)$

3601

2688

3270

4775

6295

6126

7044

5614

6515

7312

6533

6243

3754

3377

4239

4794

3971

4942

4310

3400

3999

5569

6572

6329

6057

6368

5376

7395

7568

7274

8616

7843

9200

8493

9897

9798

8623

9393

8754 $y$

$5510(20)$

$6520(30)$

$7160(30)$

$6410(20)$

6393

7260

8139

8141

7385

8088

7702

7285

7603

6681

6406

5336

5635

5417

5872

4980

4498

4234

3924

4301

4508

3652

3451

4012

4389

3669

3906

4745

4188

4927

7394

7476

7260

6692

6568

6152

5542

5507

6365 $z$

3749 (15)

4010 (18)

4590 (17)

3582 (13)

3695

3480

3060

2898

3409

2763

2801

2250

2073

2331

2026

2354

3103

2632

2662

2151

2230

2328

3083

2998

3409

2811

2890

2534

3858

3687

3614

2915

3285

3425

3806

3454

3103

2989

3609

3160

3442

3801

4202
$\mathrm{U}(\mathrm{eq})$

14 (12)

35 (16)

$42(18)$

$23(14)$

18

23

30

27

20

25

25

28

28

24

24

21

39

39

39

38

38

38

44

44

44

39

39

39

33

33

33

37

37

37

24

24

34

34

32

32

29

29

21 


\begin{tabular}{|c|c|c|c|c|}
\hline $\mathrm{H} 27$ & 8512 & 4751 & 4173 & 29 \\
\hline $\mathrm{H} 28$ & 7653 & 4117 & 4663 & 26 \\
\hline $\mathrm{H} 29$ & 6557 & 4635 & 5077 & 25 \\
\hline H31A & 5924 & 5786 & 5320 & 21 \\
\hline H31B & 5400 & 5779 & 4863 & 21 \\
\hline H33A & 7310 & 6147 & 5561 & 31 \\
\hline H33B & 7867 & 6754 & 5761 & 31 \\
\hline H33C & 7829 & 6644 & 5239 & 31 \\
\hline H34A & 5677 & 7292 & 5925 & 37 \\
\hline H34B & 6545 & 7034 & 6168 & 3 \\
\hline $\mathrm{H} 34 \mathrm{C}$ & 5920 & 6512 & 5920 & 37 \\
\hline H35A & 7323 & 7863 & 5183 & 32 \\
\hline H35B & 7380 & 7899 & 5709 & 32 \\
\hline $\mathrm{H} 35 \mathrm{C}$ & 6490 & 8101 & 5463 & 32 \\
\hline H37A & 4350 & 6300 & 5268 & 33 \\
\hline H37B & 3736 & 6945 & 5320 & 33 \\
\hline H37C & 4568 & 6852 & 5635 & 33 \\
\hline H38A & 5104 & 7972 & 5420 & 31 \\
\hline H38B & 4313 & 8086 & 5081 & 31 \\
\hline $\mathrm{H} 38 \mathrm{C}$ & 5306 & 8146 & 4915 & 31 \\
\hline H39A & 4837 & 7335 & 4330 & \\
\hline H39B & 3894 & 7242 & 4553 & 30 \\
\hline H39C & 4503 & 6603 & 4463 & 30 \\
\hline H41 & 9723 & 6100 & 6258 & 43 \\
\hline H42 & 9935 & 5761 & 5531 & 9 \\
\hline H43 & 9092 & 4881 & 5240 & - \\
\hline H44 & 8043 & 4365 & 5686 & 6 \\
\hline H45 & 7894 & 4653 & 6427 & 55 \\
\hline H46 & 8674 & 5573 & 6691 & 5 \\
\hline $\mathrm{H} 41 \mathrm{~A}$ & 8986 & 5787 & 6607 & 5 \\
\hline $\mathrm{H} 42 \mathrm{~A}$ & 9792 & 6111 & 5993 & 1 \\
\hline $\mathrm{H} 43 \mathrm{~A}$ & 9748 & 5475 & 5347 & 8 \\
\hline $\mathrm{H} 44 \mathrm{~A}$ & 8969 & 4472 & 5352 & 78 \\
\hline $\mathrm{H} 45 \mathrm{~A}$ & 8059 & 4188 & 5939 & 89 \\
\hline H46A & 8030 & 4884 & 6556 & 67 \\
\hline
\end{tabular}


Table 8 Atomic Occupancy

Atom Occupancy Atom Occupancy Atom Occupancy

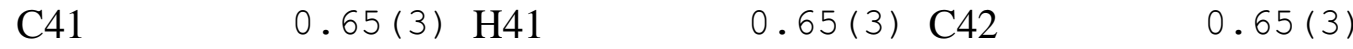

$\mathrm{H} 420.65(3) \mathrm{C} 43 \quad 0.65(3) \mathrm{H} 43 \quad 0.65(3)$

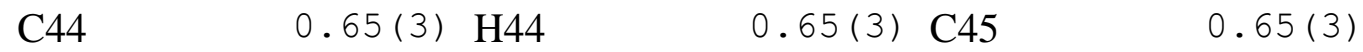

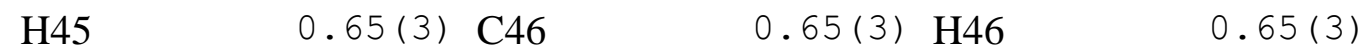

$\begin{array}{llll}\mathrm{C} 41 \mathrm{~A} & 0.35(3) \mathrm{H} 41 \mathrm{~A} & 0.35(3) \mathrm{C} 42 \mathrm{~A} & 0.35(3)\end{array}$

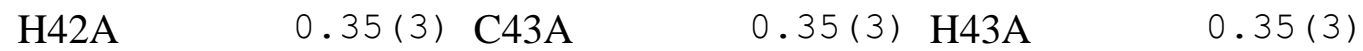

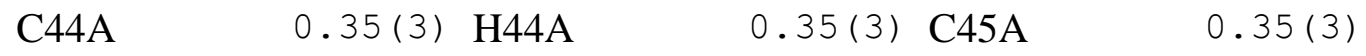

$\begin{array}{lllll}\mathrm{H} 45 \mathrm{~A} & 0.35(3) & \mathrm{C} 46 \mathrm{~A} & 0.35(3) \mathrm{H} 46 \mathrm{~A} & 0.35(3)\end{array}$ 


\author{
Prepared by \\ Louise N. Dawe, PhD \\ Department of Chemistry and Biochemistry \\ Wilfrid Laurier University \\ Science Building \\ 75 University Ave. W. \\ Waterloo, ON, ON \\ ldawe@wlu.ca
}

August 16, 2020

Introduction

Dr. Paul D. Boyle, Department of Chemistry X-Ray Facility, Western University, is acknowledged for assistance with X-ray data collection.

$\mathrm{H}$-atoms, except $\mathrm{H} 1$ and $\mathrm{H} 2$, were introduced in calculated positions and refined on a riding model. All other atoms were introduced in difference map positions and refined with fixed isotropic displacements $(\mathrm{H} 1$ and $\mathrm{H} 2)$ or anisotropically (all non-hydrogen atoms). Distance restraints were applied to two $\mathrm{C}$-C bonds (using the literature value for an average $\mathrm{sp}^{3} \mathrm{C}-\mathrm{C}$ bond). Distance restraints were applied to the $\mathrm{Ru}-\mathrm{H}$ bond lengths based on the average values resulting from two Cambridge Structural Database (CSD 5.41 with May 2020 updates); one for bridging $\mathrm{H}$ bond lengths to two $\mathrm{Ru}$ centres, and one for singly bonded $\mathrm{H}$ bond length to $\mathrm{Ru}$.

The shelxT solution was in Pca2 ${ }_{1}$, however, an alternate space group of Pbcn was suggested by validation software. When shelxS was used to solve in Pbcn two problems were observed.

1. Issues with matrix correlations were present.

2. In the Pbcn model, the bridging hydride should lie on a proper rotation axis, and generate the full dimer by a symmetry operation. The issue is that the dimer is not symmetric; one half has an agostic interaction to one of the $\mathrm{CH}_{3}$ hydrogens for the $\mathrm{Ru}$, while the other side is coordinated to a singly bonded hydride.

As a result, the structure was refined as an inversion twin in the non-centrosymmetric space group Pca $2{ }_{1}$ with mass fractions 0.57(10):0.43(10).

Several atoms in the model exhibit non-ideal ellipsoids. For this reason, the data images were processed repeatedly, looking for evidence of non-merohedral twinning, and also processed using CrysAlis as a multicrystal (that is, looking for evidence that multiple non-identical cells were present). Examination of the reflections using a reciprocal lattice viewer did indicate that there were a large number of spurious peaks, but no further twin domains, or other order associated with these peaks was detected. The ellipsoids in this model in part reflect that there were some overlapping peaks for which the contributions from these spurious peaks could not be well-resolved. The X-ray structural model is in good agreement with DFT calculation, NMR data, and 
other supporting characterizations. A global rigid bond restraint was applied to assist with a physically reasonable model with respect to ellipsoid orientation.

\section{Experimental}

A single crystal of $\mathrm{C}_{40} \mathrm{H}_{56} \mathrm{~N}_{4} \mathrm{O}_{2} \mathrm{P}_{2} \mathrm{Ru}_{2}$ was selected and collected on a Bruker APEX-II CCD diffractometer. The crystal was kept at 110(2) K during data collection. Using Olex2 [1], the structure was solved with the ShelXT [2] structure solution program using Direct Methods and refined with the ShelXL [3] refinement package using Least Squares minimisation.

\section{Crystal structure determination}

Crystal Data for $\mathrm{C}_{40} \mathrm{H}_{56} \mathrm{~N}_{4} \mathrm{O}_{2} \mathrm{P}_{2} \mathrm{Ru}_{2}(M=888.96 \mathrm{~g} / \mathrm{mol})$ : orthorhombic, space group Pca2 1 (no. 29), $a=$ 18.892(10) $\AA, b=13.920(7) \AA, c=14.896(7) \AA, V=3917(3) \AA^{3}, Z=4, T=110(2), \mu(\operatorname{MoK} \alpha)=0.892 \mathrm{~mm}^{-}$ ${ }^{1}$, Dcalc $=1.507 \mathrm{~g} / \mathrm{cm}^{3}, 149470$ reflections measured $\left(2.926^{\circ} \leq 2 \theta \leq 52.742^{\circ}\right), 7998$ unique (7565 with I $>2 \sigma(\mathrm{I})$; $\left.R_{\text {int }}=0.0350, \mathrm{R}_{\text {sigma }}=0.0129\right)$ which were used in all calculations. The final $R_{1}$ was $0.0585(\mathrm{I}>2 \sigma(\mathrm{I}))$ and $w R_{2}$ was 0.1201 (all data). 


\section{Table 1 Crystal data and structure refinement}

Identification code

Empirical formula

Formula weight

Temperature/K

Crystal system

Space group

$\mathrm{a} / \AA$

$\mathrm{b} / \AA$

$\mathrm{c} / \AA$

$\alpha /^{\circ}$

$\beta /{ }^{\circ}$

$\gamma /{ }^{\circ}$

Volume/ $/ \AA^{3}$

Z

$\rho_{\text {calc } g / \mathrm{cm}^{3}}$

$\mu / \mathrm{mm}^{-1}$

$\mathrm{F}(000)$

Crystal size $/ \mathrm{mm}^{3}$

Radiation b21014_0m (Western X-ray Code)

$\mathrm{C}_{40} \mathrm{H}_{56} \mathrm{~N}_{4} \mathrm{O}_{2} \mathrm{P}_{2} \mathrm{Ru}_{2}$

888.96

110(2)

orthorhombic

Pca2 1

18.892(10)

$13.920(7)$

$14.896(7)$

90

90

90

3917(3)

4

1.507

0.892

1832.0

$0.458 \times 0.418 \times 0.053$

$\operatorname{MoK} \alpha(\lambda=0.71073)$

$2 \theta$ range for data collection/ ${ }^{\circ} 2.926$ to 52.742

Index ranges

$-23 \leq \mathrm{h} \leq 23,-17 \leq \mathrm{k} \leq 17,-18 \leq 1 \leq 18$

Reflections collected

149470

Independent reflections

7998 [7565 with $\mathrm{I}>2 \sigma(\mathrm{I}) ; \mathrm{R}_{\text {int }}=0.0350, \mathrm{R}_{\text {sigma }}=0.0129$ ]

Data/restraints/parameters

Goodness-of-fit on $\mathrm{F}^{2}$

7998/412/470

1.150

Final $R$ indexes $[\mathrm{I}>=2 \sigma(\mathrm{I})] \quad \mathrm{R}_{1}=0.0585, \mathrm{wR}_{2}=0.1176$

Final $\mathrm{R}$ indexes [all data] $\quad \mathrm{R}_{1}=0.0624, \mathrm{wR}_{2}=0.1201$

Largest diff. peak/hole / e $\AA^{-3} 0.88 /-1.33$

Flack parameter

$0.43(10)$ 
Table 2 Fractional Atomic Coordinates $\left(\times 10^{4}\right)$ and Equivalent Isotropic Displacement Parameters $\left(\AA^{2} \times 10^{3}\right)$. Ueq is defined as $1 / 3$ of of the trace of the orthogonalised UIJ tensor.

\begin{tabular}{|c|c|c|c|c|}
\hline Atom & $x$ & $y$ & $z$ & $\mathbf{U}(\mathbf{e q})$ \\
\hline $\mathrm{Ru} 1$ & $5372.4(5)$ & $8164.6(7)$ & $6171.5(5)$ & $33.3(3)$ \\
\hline $\mathrm{Ru} 2$ & $5401.0(5)$ & $6894.1(7)$ & $4230.3(5)$ & $36.1(3)$ \\
\hline $\mathrm{P} 1$ & $4738(2)$ & $7241(2)$ & $7140(2)$ & $32.0(7)$ \\
\hline $\mathrm{P} 2$ & $4792(2)$ & $7774(2)$ & $3210(3)$ & $37.9(8)$ \\
\hline $\mathrm{O} 1$ & $4319(6)$ & $9768(7)$ & $6079(9)$ & $57(3)$ \\
\hline $\mathrm{O} 2$ & $4374(5)$ & $5261(6)$ & $4317(9)$ & $52(3)$ \\
\hline N1 & $6170(6)$ & $7207(8)$ & $6520(8)$ & $31.2(19)$ \\
\hline $\mathrm{N} 2$ & $6283(6)$ & $8842(7)$ & $5757(8)$ & $30.5(19)$ \\
\hline N3 & $6197(6)$ & $7834(7)$ & $3878(8)$ & $34.2(19)$ \\
\hline N4 & $6305(5)$ & $6146(7)$ & $4658(7)$ & $27.9(18)$ \\
\hline $\mathrm{C} 1$ & $5228(7)$ & $6114(9)$ & $7017(11)$ & $43(3)$ \\
\hline $\mathrm{C} 2$ & $6015(8)$ & $6344(10)$ & $6957(10)$ & $41(3)$ \\
\hline C3 & $6571(9)$ & $5815(11)$ & $7318(12)$ & $54(3)$ \\
\hline $\mathrm{C} 4$ & $7227(8)$ & $6074(11)$ & $7218(10)$ & $52(3)$ \\
\hline $\mathrm{C} 5$ & $7403(11)$ & $6940(12)$ & $6726(16)$ & $61(4)$ \\
\hline C6 & $6849(8)$ & 7491 (9) & $6414(9)$ & $36(2)$ \\
\hline $\mathrm{C} 7$ & $6922(7)$ & $8407(10)$ & $5968(10)$ & $41(3)$ \\
\hline $\mathrm{C} 8$ & $7554(8)$ & $8900(11)$ & $5844(10)$ & $47(3)$ \\
\hline C9 & $7548(9)$ & $9834(12)$ & $5424(13)$ & $64(4)$ \\
\hline $\mathrm{C} 10$ & $6904(8)$ & $10228(11)$ & $5208(9)$ & $46(3)$ \\
\hline C11 & $6315(8)$ & $9752(9)$ & $5376(10)$ & $36(2)$ \\
\hline C12 & $3758(7)$ & $6901(11)$ & $6905(9)$ & $49(2)$ \\
\hline C13 & $3656(7)$ & $6928(12)$ & $5878(8)$ & $59(3)$ \\
\hline $\mathrm{C} 14$ & $3271(8)$ & $7756(11)$ & $7212(10)$ & $58(3)$ \\
\hline $\mathrm{C} 15$ & $3495(8)$ & $5990(10)$ & 7269 (11) & $68(4)$ \\
\hline C16 & $4784(8)$ & $7550(9)$ & $8404(10)$ & $45(2)$ \\
\hline C17 & $4467(10)$ & $8497(10)$ & $8524(9)$ & $50(3)$ \\
\hline C18 & $4348(7)$ & $6840(9)$ & $8986(7)$ & $50(3)$ \\
\hline C19 & $5530(7)$ & $7481(8)$ & $8658(7)$ & $43(2)$ \\
\hline $\mathrm{C} 20$ & $5359(8)$ & $8880(9)$ & $3239(9)$ & $44(2)$ \\
\hline $\mathrm{C} 21$ & $6094(9)$ & $8644(9)$ & $3412(10)$ & $45(3)$ \\
\hline $\mathrm{C} 22$ & $6641(11)$ & $9189(11)$ & $3093(14)$ & $65(4)$ \\
\hline $\mathrm{C} 23$ & $7363(10)$ & $8876(13)$ & $3322(13)$ & $71(4)$ \\
\hline $\mathrm{C} 24$ & $7448(11)$ & $8077(11)$ & $3734(15)$ & $54(3)$ \\
\hline $\mathrm{C} 25$ & $6869(8)$ & $7525(9)$ & $4060(9)$ & $38(2)$ \\
\hline $\mathrm{C} 26$ & $6930(7)$ & $6577(10)$ & $4457(8)$ & $33(2)$ \\
\hline $\mathrm{C} 27$ & $7589(8)$ & $6141(13)$ & $4619(14)$ & $61(4)$ \\
\hline $\mathrm{C} 28$ & $7613(8)$ & $5264(12)$ & $4891(11)$ & $52(3)$ \\
\hline $\mathrm{C} 29$ & $6990(8)$ & $4796(11)$ & $5141(12)$ & $52(4)$ \\
\hline $\mathrm{C} 30$ & $6339(7)$ & $5280(9)$ & $5025(9)$ & $32(2)$ \\
\hline C31 & $3891(8)$ & $8209(10)$ & $3284(10)$ & $55(3)$ \\
\hline C32 & $3869(7)$ & $8985(11)$ & $4063(9)$ & $63(3)$ \\
\hline
\end{tabular}


Table 2 Fractional Atomic Coordinates $\left(\times 10^{4}\right)$ and Equivalent Isotropic Displacement Parameters $\left(\AA^{2} \times 10^{3}\right)$. Ueq is defined as $1 / 3$ of of the trace of the orthogonalised UIJ tensor.

$\begin{array}{lrrrr}\text { Atom } & \boldsymbol{x} & \boldsymbol{y} & \boldsymbol{z} & \mathbf{U}(\mathbf{e q}) \\ \text { C33 } & 3400(8) & 7421(10) & 3495(11) & 61(3) \\ \text { C34 } & 3631(9) & 8811(10) & 2443(10) & 68(4) \\ \text { C35 } & 4954(9) & 7341(9) & 2038(9) & 47(3) \\ \text { C36 } & 4509(10) & 6504(10) & 1709(9) & 48(3) \\ \text { C37 } & 5103(11) & 8074(10) & 1319(9) & 83(4) \\ \text { C38 } & 5697(8) & 6740(11) & 2062(9) & 64(3) \\ \text { C39 } & 4720(6) & 9131(9) & 6093(9) & 32(2) \\ \text { C40 } & 4761(7) & 5905(9) & 4358(11) & 39(3)\end{array}$


Table 3 Anisotropic Displacement Parameters $\left(\AA^{2} \times 10^{3}\right)$. The Anisotropic displacement factor exponent takes the form: $-2 \pi^{2}\left[h^{2} a^{* 2} U_{11}+2 h k a * b * U_{12}+\ldots\right]$.

\begin{tabular}{|c|c|c|c|c|c|c|}
\hline Atom & $\mathrm{U}_{11}$ & $\mathbf{U}_{22}$ & $\mathbf{U}_{33}$ & $\mathbf{U}_{23}$ & $\mathbf{U}_{13}$ & $\mathbf{U}_{12}$ \\
\hline Ru1 & $26.3(5)$ & $38.9(5)$ & $34.8(6)$ & $17.6(5)$ & $-7.5(5)$ & $-8.8(4)$ \\
\hline $\mathrm{Ru} 2$ & $28.1(5)$ & $39.2(5)$ & $40.9(7)$ & $20.3(5)$ & $12.3(5)$ & $12.1(4)$ \\
\hline $\mathrm{P} 1$ & $55(2)$ & $25.1(15)$ & $16.5(15)$ & $-8.4(12)$ & $7.7(14)$ & $-11.5(14)$ \\
\hline $\mathrm{P} 2$ & $65(2)$ & $21.4(15)$ & $27.3(18)$ & $5.2(14)$ & $1.2(17)$ & $10.6(15)$ \\
\hline $\mathrm{O} 1$ & $56(5)$ & $53(5)$ & $61(7)$ & $1(6)$ & $-21(6)$ & $7(4)$ \\
\hline $\mathrm{O} 2$ & $52(5)$ & $40(4)$ & $64(7)$ & $-5(5)$ & $-3(6)$ & $-6(3)$ \\
\hline N1 & $41(3)$ & $28(4)$ & $25(5)$ & $-2(3)$ & $3(4)$ & $-1(3)$ \\
\hline $\mathrm{N} 2$ & $37(3)$ & $28(3)$ & $27(5)$ & $-3(3)$ & $2(4)$ & $-4(3)$ \\
\hline N3 & $53(4)$ & $24(3)$ & $26(5)$ & $-11(3)$ & $4(4)$ & $-5(3)$ \\
\hline N4 & $27(3)$ & $33(3)$ & $24(5)$ & $-4(3)$ & $6(3)$ & $10(3)$ \\
\hline $\mathrm{C} 1$ & $64(4)$ & $21(5)$ & $45(8)$ & $-9(4)$ & $14(5)$ & $-8(4)$ \\
\hline $\mathrm{C} 2$ & $60(4)$ & $30(4)$ & $32(6)$ & $5(4)$ & $15(4)$ & $5(3)$ \\
\hline C3 & $75(4)$ & $45(6)$ & $42(8)$ & $9(5)$ & $20(5)$ & $25(4)$ \\
\hline $\mathrm{C} 4$ & $71(4)$ & $53(6)$ & $32(6)$ & $3(4)$ & $18(5)$ & $30(5)$ \\
\hline $\mathrm{C} 5$ & $50(4)$ & $69(7)$ & $63(10)$ & $20(7)$ & $5(6)$ & $20(5)$ \\
\hline C6 & $38(3)$ & $40(4)$ & $30(6)$ & $3(4)$ & $5(4)$ & $3(3)$ \\
\hline $\mathrm{C} 7$ & $35(3)$ & $41(4)$ & $46(8)$ & $7(5)$ & $3(4)$ & $-3(3)$ \\
\hline $\mathrm{C} 8$ & $42(4)$ & $71(6)$ & $28(6)$ & $11(5)$ & $-2(5)$ & $-19(4)$ \\
\hline C9 & $54(5)$ & $72(6)$ & $66(11)$ & $22(7)$ & $-17(6)$ & $-35(5)$ \\
\hline $\mathrm{C} 10$ & $58(5)$ & $57(6)$ & $23(6)$ & $8(5)$ & $-7(5)$ & $-27(4)$ \\
\hline C11 & $48(4)$ & $27(4)$ & $32(6)$ & $-2(4)$ & $-1(5)$ & $-9(3)$ \\
\hline $\mathrm{C} 12$ & $45(5)$ & $56(5)$ & $45(5)$ & $-14(4)$ & $8(4)$ & $-26(4)$ \\
\hline C13 & $43(7)$ & $89(9)$ & $46(5)$ & $-15(5)$ & $0(4)$ & $-28(6)$ \\
\hline C14 & $51(7)$ & $71(6)$ & $51(7)$ & $-5(6)$ & $-5(6)$ & $-3(5)$ \\
\hline C15 & $61(8)$ & $61(6)$ & $81(9)$ & $3(6)$ & $-1(7)$ & $-30(5)$ \\
\hline C16 & $63(5)$ & $39(5)$ & $34(5)$ & $-11(4)$ & $3(3)$ & $5(4)$ \\
\hline C17 & $87(8)$ & $50(5)$ & $14(5)$ & $-6(5)$ & $10(6)$ & $22(6)$ \\
\hline C18 & $71(6)$ & $51(5)$ & $29(5)$ & $-7(4)$ & $8(5)$ & $2(5)$ \\
\hline C19 & $66(5)$ & $41(6)$ & $21(5)$ & $-6(4)$ & $1(4)$ & $2(4)$ \\
\hline $\mathrm{C} 20$ & $86(4)$ & $31(5)$ & $16(5)$ & $-6(4)$ & $-15(4)$ & $-3(4)$ \\
\hline $\mathrm{C} 21$ & $82(4)$ & $21(4)$ & $33(6)$ & $-10(4)$ & $-13(5)$ & $-10(3)$ \\
\hline $\mathrm{C} 22$ & $95(5)$ & $33(6)$ & $66(10)$ & $2(6)$ & $-16(6)$ & $-26(5)$ \\
\hline $\mathrm{C} 23$ & $87(5)$ & $66(6)$ & $58(10)$ & $12(6)$ & $-20(7)$ & $-38(5)$ \\
\hline $\mathrm{C} 24$ & $60(4)$ & $64(6)$ & $39(7)$ & $2(5)$ & $1(5)$ & $-23(5)$ \\
\hline $\mathrm{C} 25$ & $49(4)$ & $42(4)$ & $22(5)$ & $-8(4)$ & $3(4)$ & $-7(3)$ \\
\hline $\mathrm{C} 26$ & $31(3)$ & $49(4)$ & $17(5)$ & $-5(4)$ & $7(3)$ & $1(3)$ \\
\hline $\mathrm{C} 27$ & $29(4)$ & $73(6)$ & $80(12)$ & $19(7)$ & $3(6)$ & $3(4)$ \\
\hline C28 & $36(4)$ & $76(6)$ & $44(8)$ & $16(6)$ & $4(5)$ & $12(4)$ \\
\hline C29 & $39(4)$ & $49(6)$ & $68(11)$ & $14(6)$ & $10(5)$ & $19(4)$ \\
\hline $\mathrm{C} 30$ & $35(4)$ & $36(4)$ & $27(6)$ & $-2(4)$ & $5(5)$ & $11(3)$ \\
\hline C31 & $64(5)$ & $60(6)$ & $40(5)$ & $-11(4)$ & $-19(4)$ & $23(4)$ \\
\hline C32 & $62(8)$ & $76(7)$ & $50(6)$ & $-21(6)$ & $-7(6)$ & $31(6)$ \\
\hline
\end{tabular}


Table 3 Anisotropic Displacement Parameters $\left(\AA^{2} \times 10^{3}\right)$. The Anisotropic displacement factor exponent takes the form: $-2 \pi^{2}\left[h^{2} a^{* 2} U_{11}+2 h k a * b * U_{12}+\ldots\right]$.

\begin{tabular}{|c|c|c|c|c|c|c|}
\hline Atom & $\mathrm{U}_{11}$ & $\mathbf{U}_{22}$ & $\mathbf{U}_{33}$ & $\mathbf{U}_{23}$ & $\mathbf{U}_{13}$ & $\mathbf{U}_{12}$ \\
\hline C33 & $61(7)$ & $64(6)$ & $58(9)$ & $5(5)$ & $-9(6)$ & $29(5)$ \\
\hline C34 & $95(10)$ & $51(7)$ & $58(6)$ & $-1(5)$ & $-38(7)$ & $5(7)$ \\
\hline $\mathrm{C} 35$ & $83(7)$ & $42(5)$ & $16(4)$ & $-8(3)$ & $12(4)$ & $-24(4)$ \\
\hline C36 & $85(7)$ & $35(5)$ & $22(6)$ & $0(4)$ & $-13(6)$ & $-13(5)$ \\
\hline C37 & $166(13)$ & $50(6)$ & $31(6)$ & $1(5)$ & $18(7)$ & $-37(7)$ \\
\hline C38 & $83(6)$ & $74(8)$ & $36(6)$ & $-28(5)$ & $8(5)$ & $-13(6)$ \\
\hline C39 & $35(4)$ & $46(5)$ & $13(5)$ & $15(4)$ & $-5(4)$ & $-6(4)$ \\
\hline $\mathrm{C} 40$ & $32(4)$ & $31(4)$ & $53(10)$ & $-6(5)$ & $0(6)$ & $8(3)$ \\
\hline
\end{tabular}


Table 4 Bond Lengths.

\begin{tabular}{|c|c|c|c|c|c|}
\hline \multicolumn{2}{|c|}{ Atom Atom } & \multirow{2}{*}{$\begin{array}{c}\text { Length/Å } \\
2.275(4)\end{array}$} & \multicolumn{2}{|c|}{ Atom Atom } & \multirow{2}{*}{$\begin{array}{c}\text { Length/̊̊ } \\
1.38(2)\end{array}$} \\
\hline Ru1 & P1 & & $\mathrm{C} 5$ & C6 & \\
\hline a1 & $\mathrm{N} 1$ & $2.077(11)$ & C6 & C7 & $45(17)$ \\
\hline 11 & $\mathrm{~N} 2$ & $58(11)$ & C7 & & $.388(19)$ \\
\hline 11 & C39 & (13) & $\mathrm{C} 8$ & & \\
\hline & P2 & $2.266(4)$ & C9 & & \\
\hline u2 & N3 & 061 (11) & $\mathrm{C} 10$ & & .02 \\
\hline $\mathrm{u} 2$ & N4 & $100(10)$ & $\mathrm{C} 12$ & & .54 \\
\hline $\mathrm{u} 2$ & $\mathrm{C} 40$ & $843(13)$ & $\mathrm{C} 12$ & & \\
\hline 1 & $\mathrm{C} 1$ & 13) & $\mathrm{C} 12$ & & .4 \\
\hline 1 & $\mathrm{C} 12$ & 14) & $\mathrm{C} 16$ & $\mathrm{C}$ & 15) \\
\hline 1 & $\mathrm{C} 16$ & 15) & $\mathrm{C} 16$ & $\mathrm{C}$ & 18) \\
\hline 2 & $\mathrm{C} 2$ & 4) & $\mathrm{C} 16$ & & 1 \\
\hline 2 & $\mathrm{C} 3$ & 5) & $\mathrm{C} 20$ & & \\
\hline 2 & $\mathrm{C} 3$ & 1.8 & $\mathrm{C} 21$ & C & \\
\hline 1 & $\mathrm{C}^{3}$ & 1.1 & $\mathrm{C} 22$ & & \\
\hline 2 & $\mathrm{C} 4$ & 1.1 & $\mathrm{C} 23$ & & \\
\hline 1 & $\mathrm{C} 2$ & $1.397(17)$ & $\mathrm{C} 24$ & & $.42(2)$ \\
\hline 1 & $\mathrm{Ct}$ & $1.3 !$ & $\mathrm{C} 25$ & $\mathrm{C}$ & $.450(17)$ \\
\hline 2 & $\mathrm{C} 7$ & 1.38 & $\mathrm{C} 26$ & $\mathrm{C}$ & 141 \\
\hline 2 & $\mathrm{C} 1$ & 1.3 & $\mathrm{C} 27$ & C & 1.29 \\
\hline 3 & $\mathrm{C} 2$ & 1.3 & $\mathrm{C} 28$ & $\mathrm{C}^{\prime}$ & 1 \\
\hline 13 & $\mathrm{C} 2$ & 1.3 & $\mathrm{C} 29$ & $\mathrm{C}$ & 11 \\
\hline J4 & $\mathrm{C} 2$ & 1.3 & C31 & $\mathrm{C}$ & 1.58 \\
\hline N4 & $\mathrm{C} 30$ & $1.325(17)$ & C31 & $\mathrm{C} 33$ & $1.470(18$ \\
\hline $\mathrm{C1}$ & $\mathrm{C} 2$ & $1.522(19)$ & C 31 & C34 & $1.586(18)$ \\
\hline 22 & $\mathrm{C} 3$ & $1.39(2)$ & C35 & C36 & $1.519(18)$ \\
\hline 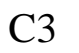 & $\mathrm{C} 4$ & $0(2)$ & C35 & C37 & $1.505(17)$ \\
\hline $\mathrm{C} 4$ & $\mathrm{C} 5$ & $1.45(2)$ & $\mathrm{C} 35$ & C38 & $1.63(2)$ \\
\hline
\end{tabular}


Table 5 Bond Angles.

\section{Atom Atom Atom}

N1 Ru1 P1

N2 Ru1 P1

N2 Ru1 N1

C39 Ru1 P1

C39 Ru1 N1

C39 Ru1 N2

N3 Ru2 P2

N3 Ru2 N4

N4 Ru2 P2

C40 Ru2 P2

C40 Ru2 N3

C40 Ru2 N4

C1 P1 Ru1

C1 P1 C12

C1 P1 C16

C12 P1 Ru1

C16 P1 Ru1

C16 P1 C12

C20 P2 Ru2

C31 P2 Ru2

C31 P2 C20

C31 P2 C35

C35 P2 Ru2

C35 P2 C20

C2 N1 Ru1

C6 N1 Ru1

C6 N1 C2

C7 N2 Ru1

C7 N2 $\quad$ C11

C11 N2 Ru1

C21 N3 Ru2

C21 N3 C25

C25 N3 Ru2

C26 N4 Ru2

C30 N4 Ru2

C30 N4 C26

C2 $\mathrm{C} 1 \quad \mathrm{P} 1$

N1 C2 C1

C3 C2 N1

C3 32 C1

C4 4 C3 22

C3 $34 \quad \mathrm{C} 5$

C6 5 C5 $\mathrm{C} 4$
Angle $/^{\circ}$

Atom Atom Atom

$82.0(3) \quad \mathrm{C} 8 \quad \mathrm{C} 7 \quad \mathrm{C} 6$

153.0(3) C7 C8 C9

76.3(4) C10 C9 C8

95.8(4) C11 C10 C9

167.7(5) C10 C11 N2

102.0 (5) C13 C12 P1

81.7(4) $\mathrm{C} 13 \quad \mathrm{C} 12 \quad \mathrm{C} 14$

78.4(4) C14 C12 P1

152.2(3) C15 C12 P1

$98.0(5) \quad \mathrm{C} 15 \quad \mathrm{C} 12 \quad \mathrm{C} 13$

168.4(5) C15 C12 $\mathrm{C} 14$

$97.6(5) \quad \mathrm{C} 17 \quad \mathrm{C} 16 \quad \mathrm{P} 1$

98.8 (5) $\mathrm{C} 17 \quad \mathrm{C} 16 \quad \mathrm{C} 18$

104.8(6) $\mathrm{C} 17 \quad \mathrm{C} 16 \quad \mathrm{C} 19$

105.4(7) C18 C16 P1

121.7(5) C19 C16 P1

117.9(4) C19 C16 C18

105.8(6) C21 C20 P2

97.9(5) N3 C21 C20

128.1(5) N3 C21 C22

105.1(7) C22 C21 C20

108.6(7) C21 C22 C23

111.6(5) C24 C23 C22

101.1(6) C23 C24 C25

121.1(9) N3 C25 C24

$118.1(9) \quad \mathrm{N} 3 \quad \mathrm{C} 25 \quad \mathrm{C} 26$

120.3(12) $\mathrm{C} 24 \quad \mathrm{C} 25 \quad \mathrm{C} 26$

117.4(9) N4 C26 C25

$117.0(11)$ N4 C26 C27

125.2(9) $\quad$ C27 $\quad$ C26 $\quad$ C25

124.1(10) $\mathrm{C} 28 \quad \mathrm{C} 27 \quad \mathrm{C} 26$

120.2(13) $\mathrm{C} 27 \quad \mathrm{C} 28 \quad \mathrm{C} 29$

115.3(9) $\mathrm{C} 28 \quad \mathrm{C} 29 \quad \mathrm{C} 30$

$114.9(9) \quad \mathrm{N} 4 \quad \mathrm{C} 30 \quad \mathrm{C} 29$

128.0(9) C32 C31 P2

116.9(11) C33 C31 P2

108.7(9) $\quad$ C33 $\quad$ C31 $\quad$ C32

114.3(12) C33 C31 C34

118.4(13) C34 C31 P2

127.1(13) C34 C31 C32

122.0 (15) $\mathrm{C} 36 \quad \mathrm{C} 35 \quad \mathrm{P} 2$

120.6(15) C36 C35 C38

117.3(17) $\mathrm{C} 37$ C35 P2
Angle $/^{\circ}$

$125.4(14)$

$119.7(15)$

$117.9(14)$

$120.2(14)$

$124.9(14)$

$107.0(9)$

$101.3(13)$

$108.8(9)$

$117.8(11)$

$110.2(12)$

$110.5(13)$

$107.6(10)$

$106.8(13)$

$115.0(13)$

$112.2(9)$

$106.3(9)$

$108.9(12)$

$111.4(9)$

$114.9(13)$

$122.5(16)$

$122.5(14)$

$117.1(16)$

$119.0(17)$

$122(2)$

$118.4(14)$

116.1 (12)

$124.8(15)$

$115.0(12)$

122.7 (13)

$122.2(14)$

$119.7(16)$

119.7 (15)

$118.6(14)$

$121.7(13)$

$107.3(10)$

$110.9(10)$

$109.6(13)$

$111.5(12)$

$114.8(11)$

102.1 (11)

$117.2(10)$

95.1 (11)

$118.4(9)$ 
Table 5 Bond Angles.

Atom Atom Atom

N1 C6 C5

Angle ${ }^{\circ}$

121.3 (13)

Atom Atom Atom

N1 C6 C7

C5 $\quad$ C6 $\quad$ C7

N2 C7 C6

N2 $\quad$ C7 $\quad$ C8
113.7(12) $\mathrm{C} 37 \quad \mathrm{C} 35 \quad \mathrm{C} 38$

125.0(14) $\mathrm{C} 38$ C35 P2

$114.0(12)$ O1 C39 Ru1

120.1(13) O2 C40 Ru2
Angle/ ${ }^{\circ}$

113.2 (12)

$101.8(12)$

$106.5(9)$

$176.7(13)$

$170.8(15)$ 
Table 6 Torsion Angles.

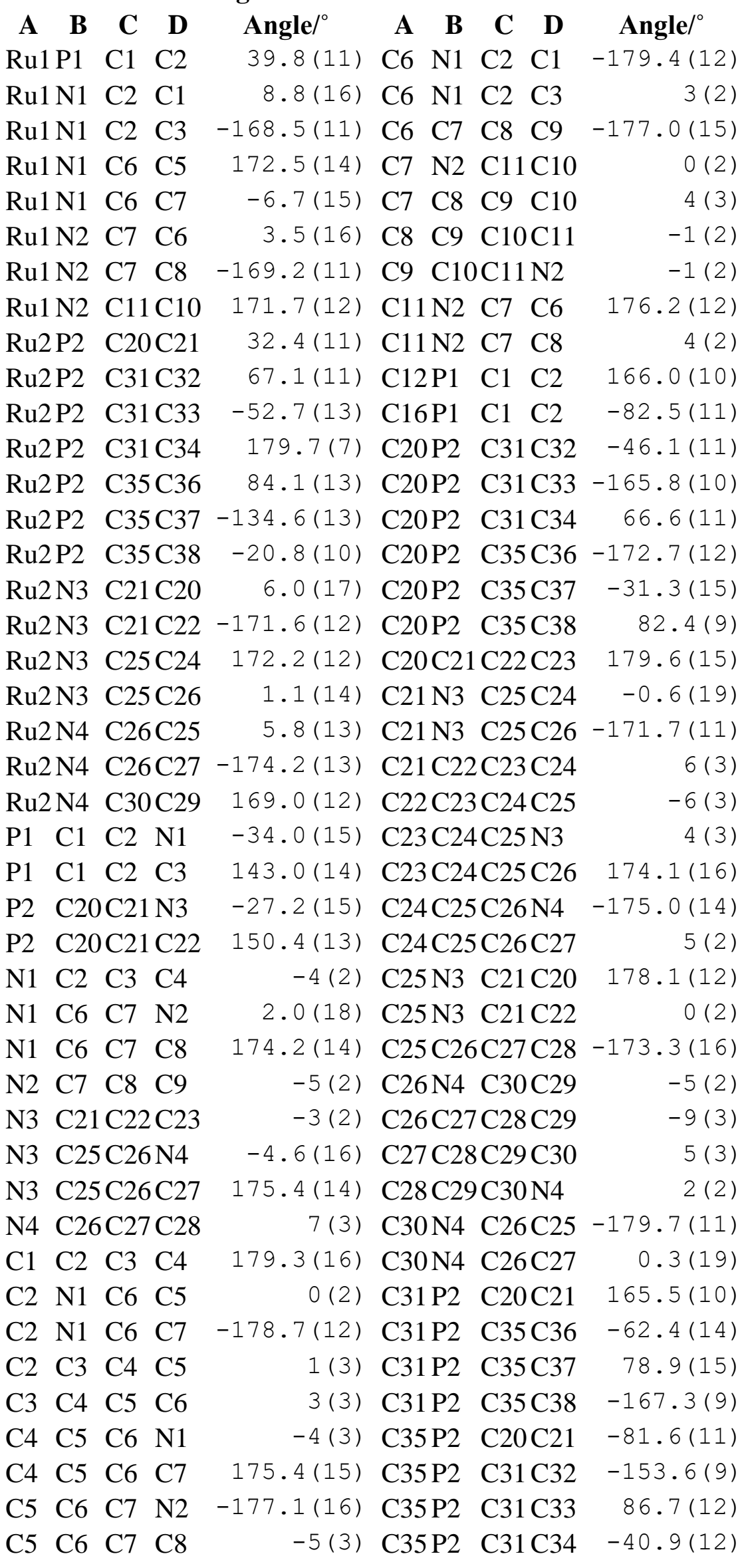


Table 7 Hydrogen Atom Coordinates $\left(\AA \times 10^{4}\right)$ and Isotropic Displacement Parameters $\left(\AA^{2} \times 10^{3}\right)$.

\begin{tabular}{|c|c|c|c|c|}
\hline Atom & $x$ & $y$ & $z$ & $\mathbf{U}(\mathbf{e q})$ \\
\hline H1 & $5080(30)$ & $7360(70)$ & $5280(40)$ & \\
\hline $\mathrm{H} 2$ & $5730(60)$ & $8510(80)$ & $7050(50)$ & \\
\hline $\mathrm{H} 1 \mathrm{~A}$ & 5136.74 & 5691.67 & 7538.31 & \\
\hline H1B & 5072.82 & 5775.24 & 6466.55 & \\
\hline $\mathrm{H} 3$ & 6468.5 & 5247.84 & 7648.01 & \\
\hline $\mathrm{H} 4$ & 7593.93 & 5692.75 & 7468.44 & \\
\hline H5 & 7881.14 & 7120.82 & 6622.1 & \\
\hline H8 & 7987.89 & 8623.06 & 6035.01 & \\
\hline H9 & 7976.16 & 10166.56 & 5300.5 & \\
\hline H10 & 6882.81 & 10844.67 & 4936.25 & \\
\hline H11 & 5879.77 & 10054.1 & 5226.4 & \\
\hline H13A & 3854.12 & 7526.93 & 5639.41 & \\
\hline H13B & 3150.39 & 6895.56 & 5737.18 & \\
\hline $\mathrm{H} 13 \mathrm{C}$ & 3900.25 & 6379.54 & 5605.71 & \\
\hline $\mathrm{H} 14 \mathrm{~A}$ & 3318.94 & 7850.13 & 7860.8 & \\
\hline H14B & 2777.21 & 7607.2 & 7066.68 & \\
\hline $\mathrm{H} 14 \mathrm{C}$ & 3413.61 & 8344.27 & 6898.24 & \\
\hline $\mathrm{H} 15 \mathrm{~A}$ & 3731.76 & 5454.35 & 6964.74 & \\
\hline H15B & 2983.5 & 5946.1 & 7170.87 & \\
\hline $\mathrm{H} 15 \mathrm{C}$ & 3595.31 & 5960.26 & 7913.81 & \\
\hline H17A & 4592.04 & 8746.89 & 9118.54 & \\
\hline H17B & 3950.97 & 8445.49 & 8475.23 & \\
\hline $\mathrm{H} 17 \mathrm{C}$ & 4644.76 & 8933.63 & 8060.59 & \\
\hline H18A & 4529.75 & 6844.77 & 9602.19 & \\
\hline H18B & 4389.73 & 6190.82 & 8736.63 & \\
\hline $\mathrm{H} 18 \mathrm{C}$ & 3849.74 & 7035.21 & 8986.75 & \\
\hline H19A & 5816.71 & 7867.5 & 8245.04 & \\
\hline H19B & 5682.52 & 6808.95 & 8628.08 & \\
\hline H19C & 5592.01 & 7721.13 & 9271.81 & \\
\hline $\mathrm{H} 20 \mathrm{~A}$ & 5321.88 & 9219.28 & 2656.84 & \\
\hline $\mathrm{H} 20 \mathrm{~B}$ & 5184.21 & 9317.19 & 3713.83 & \\
\hline $\mathrm{H} 22$ & 6559.18 & 9746.22 & 2739.83 & \\
\hline $\mathrm{H} 23$ & 7759.41 & 9260.79 & 3165.85 & \\
\hline $\mathrm{H} 24$ & 7914.92 & 7844.46 & 3826.26 & \\
\hline $\mathrm{H} 27$ & 8014.18 & 6491.34 & 4527.72 & \\
\hline $\mathrm{H} 28$ & 8053.49 & 4935.86 & 4921.03 & \\
\hline H29 & 7002.98 & 4165.65 & 5384.62 & \\
\hline H30 & 5914.11 & 4976.85 & 5215.12 & \\
\hline $\mathrm{H} 32 \mathrm{~A}$ & 4020.17 & 8688.35 & 4628.15 & \\
\hline H32B & 3385.89 & 9230.39 & 4127.62 & \\
\hline $\mathrm{H} 32 \mathrm{C}$ & 4188.94 & 9516.59 & 3914.48 & \\
\hline
\end{tabular}


Table 7 Hydrogen Atom Coordinates $\left(\AA \times 10^{4}\right)$ and Isotropic Displacement Parameters $\left(\AA^{2} \times 10^{3}\right)$.

Atom

$\mathrm{H} 33 \mathrm{~A}$

$\mathrm{H} 33 \mathrm{~B}$

$\mathrm{H} 33 \mathrm{C}$

$\mathrm{H} 34 \mathrm{~A}$

H34B

$\mathrm{H} 34 \mathrm{C}$

$\mathrm{H} 36 \mathrm{~A}$

H36B

$\mathrm{H} 36 \mathrm{C}$

H37A

H37B

H37C

H38A

H38B

H38C

\section{$x$}

3286.46

2964.58

3621.46

3964.86

3161.01

3607.16

4803.95

4118.89

4316.63

4723.08

5125.72

5555.28

6093.52

5750.65

5690.66 $y$

7068.52

7685.95

6984.45

9336.08

9077.86

8392.14

6070.79

6746.94

6154.35

8554.38

7754.81

8391.36

7186.92

6378.4

6292.29 $z$

2943.78

3752.88

3927.4

2327.94

2565.99

1915.27

1349.55

1339.03

2224.82

1312.5

733.59

1443.21

2129.69

1501.79

2570.39
$\mathrm{U}(\mathrm{eq})$

92

92

92

102

102

102

71

71

71

124

124

124

96

96

96

References

1. Dolomanov, O. V.; Bourhis, L. J.; Gildea, R. J.; Howard, J. A. K.; Puschmann, H. OLEX2: a Complete Structure Solution, Refinement and Analysis Program. J. Appl. Crystallogr. 2009, 42, 339-341.

2. Sheldrick, G. M. SHELXT - Integrated Space-Group and Crystal-Structure Determination. Acta Crystallogr., Sect. A: Found. Adv.. 2015, 71, 3-8.

3. Sheldrick, G. M. Crystal Structure Refinement with SHELXL. Acta Crystallogr., Sect. C: Struct. Chem. 2015, 71, 3-8. 